NISTIR 85-3273-22

(Rev. 5/07)

\title{
Energy Price Indices and Discount Factors for Life-Cycle Cost Analysis - April 2007
}

Annual Supplement to NIST Handbook 135 and NBS Special Publication 709
Amy S. Rushing

Barbara C. Lippiatt

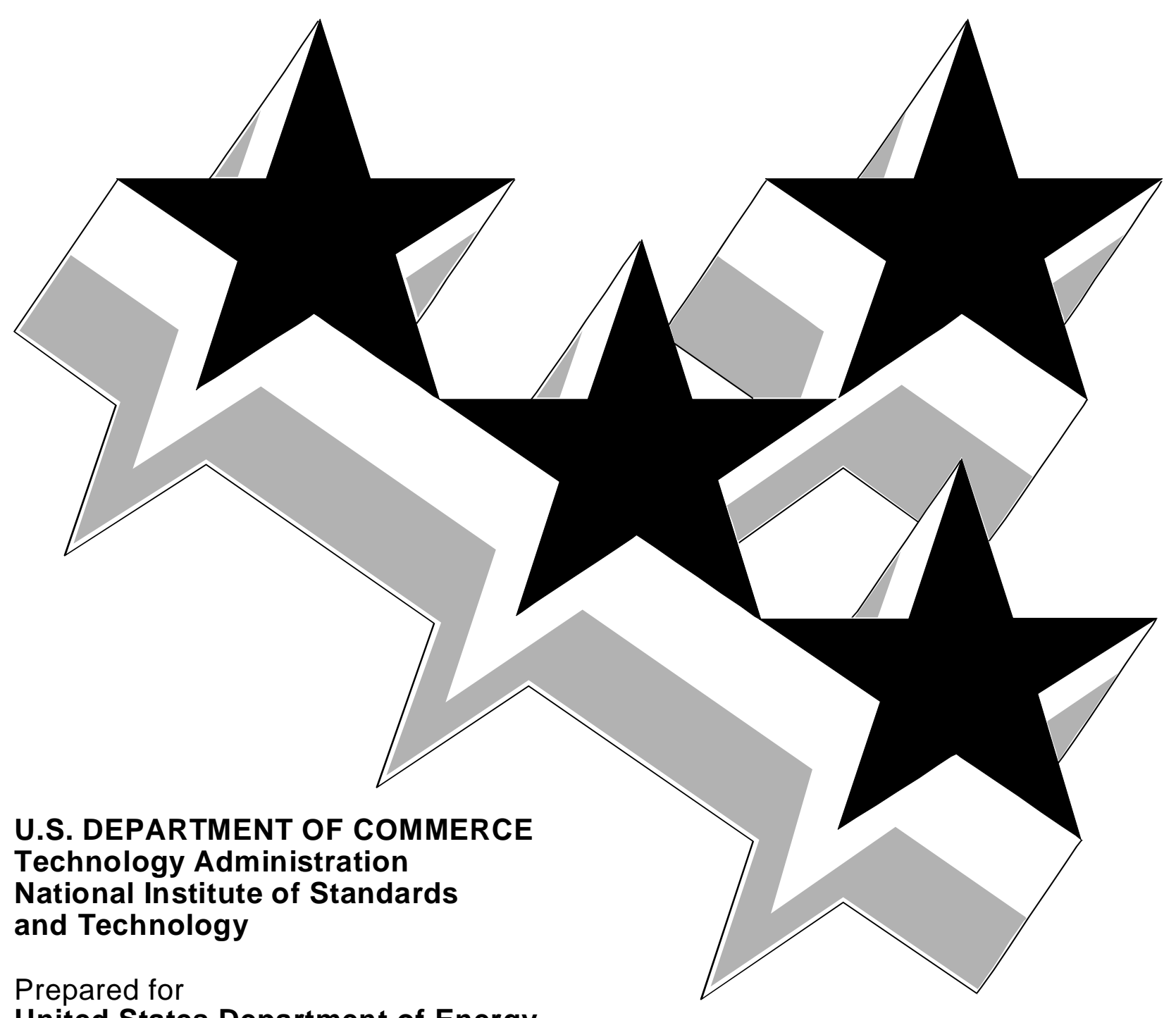

United States Department of Energy Federal Energy Management Program

May 2007 

U.S. Department of Commerce

Technology Administration

National Institute of Standards and Technology
Office of Applied Economics

Building and Fire Research Laboratory

Gaithersburg, MD 20899

\section{ENERGY PRICE INDICES AND DISCOUNT FACTORS} FOR LIFE-CYCLE COST ANALYSIS

Annual Supplement to NIST Handbook 135 and NBS Special Publication 709

\section{April 1, 2007 to March 31, 2008}

Data for the Federal Methodology for Life-Cycle Cost Analysis, Title 10, CFR, Part 436, Subpart A; and for the Energy Conservation Mandatory Performance Standards for New Federal Residential Buildings, Title 10, CFR, Part 435

\section{Amy S. Rushing \\ Barbara C. Lippiatt}

Sponsored by:

U.S. Department of Energy

Federal Energy Management Program

Washington, DC 20585

May 2007

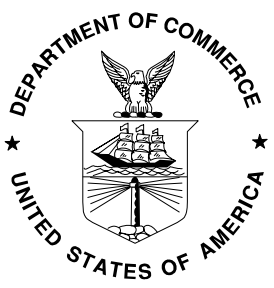

U.S. DEPARTMENT OF COMMERCE

Carlos M. Gutierrez, Secretary

TECHNOLOGY ADMINSTRATION

Robert C. Cresanti, Under Secretary for Technology

NATIONAL INSTITUTE OF STANDARDS AND TECHNOLOGY

William A. Jeffrey, Director 



\begin{abstract}
This is the April 2007 edition of energy price indices and discount factors for performing life-cycle cost analyses of energy and water conservation and renewable energy projects in federal facilities. It will be effective from April 1, 2007 to March 31, 2008. This publication supports the federal lifecycle costing methodology described in 10 CFR 436A and OMB Circular A-94 by updating the energy price projections and discount factors that are described, explained, and illustrated in NIST Handbook 135 (HB 135, Life-Cycle Costing Manual for the Federal Energy Management Program.) It supports private-sector life-cycle cost analysis by updating the energy price indices that are described, explained, and illustrated in NBS Special Publication 709 (SP 709).
\end{abstract}

\title{
Disclaimer:
}

Certain trade names or company products are mentioned in the text to specify adequately the software and operating systems used for performing the life-cycle cost analyses. In no case does such identification imply recommendation or endorsement by the National Institute of Standards and Technology, nor does it imply that the software and operating systems are the best available for the purpose. 


\section{PREFACE}

This is the April 2007 Annual Supplement to NIST Handbook 135, Life-Cycle Costing Manual for the Federal Energy Management Program (FEMP). The annual supplement provides energy price indices and discount factors for use with the Federal Energy Management Program's procedures for life-cycle cost analysis, as established by the U.S. Department of Energy (DOE) in Subpart A of Part 436 of Title 10 of the Code of Federal Regulations (10 CFR 436A), and amplified in NIST Handbook 135. These indices and factors are provided as an aid to implementing life-cycle cost evaluations of potential energy and water conservation and renewable energy investments in existing and new federally owned and leased buildings.

The update of the Annual Supplement to Handbook 135 is released on April 1 of each year. Its price indices and discount factors are calculated with the most recent energy price projections from DOE's Energy Information Administration (EIA) and the most recent discount rates from FEMP and the Office of Management and Budget (OMB) Circular A-94. This issue of the Annual Supplement is intended for use from April 1, 2007 to March 31, 2008. The updated editions of the NIST Building Life-Cycle Cost (BLCC) programs are released at the same time as this Annual Supplement, for use over the same time period. The software products are discussed below.

At the request of a number of users, a text file of the EIA energy price projections underlying this Annual Supplement has been made available by NIST. For information on obtaining this file (ENCOST07.txt) contact the authors via e-mail at blippiatt@nist.gov or amy.rushing@nist.gov.

The life-cycle costing methods and procedures, as set forth in 10 CFR 436A, are to be followed by all federal agencies, unless specifically exempted, for evaluating the cost effectiveness of potential energy and water conservation and renewable energy investments in federally owned and leased buildings. For most other federal LCC analyses OMB Circular A-94 provides the relevant guidelines.

As called for by legislation (Energy Policy and Conservation Act, P.L.94-163, 1975, 92 Stat 3206, 42 USC 8252 et seq), the National Institute of Standards and Technology has provided technical assistance to the U.S. Department of Energy in the development and implementation of life-cycle costing methods and procedures. This is the second of a three-volume set, which together provide the methods, data, and computational tools for federal life-cycle cost analysis.

Included in the three-volume set for federal life-cycle cost analysis are the following:

Life-Cycle Costing Manual for the Federal Energy Management Program, National Institute of Standards and Technology, Handbook 135 (1995).

The manual is a guide to understanding life-cycle costing and related methods of economic analysis as they are applied to federal decisions, especially those subject to 10 CFR 436A rules. It describes the required procedures and assumptions, defines and explains how to apply and interpret economic performance measures, gives examples of federal decision problems and their solutions, explains how to use energy price indices and discount factors, and provides worksheets and other computational aids and instructions for calculating the required measures. The 1995 edition of Handbook 135 is a complete revision of the 1987 edition, with updated information on the FEMP LCC requirements of 10 CFR 436A. 
(2) Energy Price Indices and Discount Factors for Life-Cycle Cost Analysis, Annual Supplement to NIST Handbook 135 and NBS Special Publication 709, National Institute of Standards and Technology, NISTIR 85-3273.

This report, which is updated annually, provides the current DOE and OMB discount rates, projected energy price indices, and corresponding discount factors needed to estimate the present values of future energy and non-energy-related project costs. Request the latest edition when ordering. (This is the report that you are holding in your hands.)

(3) NIST "Building Life-Cycle Cost" (BLCC) Computer Programs, BLCC5 and BLCC4, National Institute of Standards and Technology. These programs use as default values the same discount factors and energy price projections that underlie the discount factor tables in the Annual Supplement. Use versions BLCC 5.3-07 and BLCC 4.9-07 for the period from April 1, 2007 to March 31, 2008.

The BLCC5 program is a windowed version of the DOS-based BLCC4. It is programmed in Java and uses an xml file format, making it platform-independent. The system requirements are those of Java. They include Microsoft Windows 95, 98, NT4.0 with Service Pack 5, ME, 2000 Professional, or XP operating system. A Mac OS X installer is available upon request.

The BLCC5 User's Guide is part of its Help system. BLCC 5.3 has six modules, all of them consistent with the life-cycle cost methodology of 10 CFR 436A, but programmed to include default inputs and nomenclature for specific uses:

(1) FEMP Analysis, Energy Project

for energy and water conservation and renewable energy projects under the FEMP rules, agency-funded;

(2) Federal Analysis, Financed Project

for federal projects financed through Energy Savings Performance Contracts (ESPC) or Utility Energy Services Contracts (UESC) as authorized by Executive Order 13123 (6/99);

(3) OMB Analysis, Federal Analysis, Projects subject to OMB Circular A-94

for projects subject to OMB Circular A-94 (most other, non-energy, federal government construction projects, but not water resource projects);

(4) MILCON Analysis, Energy Project

for energy and water conservation and renewable energy projects in military construction, agency-funded;

(5) MILCON Analysis, ECIP Project

for energy and water conservation projects under the Energy Conservation Investment Program (ECIP).

(6) MILCON Analysis, Non-Energy Project

for military construction designs that are not primarily for energy or water conservation. 
The BLCC4 program is designed to run on IBM PC and compatible microcomputers in a MS-DOS environment. The current version is BLCC 4.9-07. BLCC4-associated programs are the following:

- $\quad$ QuickBLCC (version 2.9-07) can be used to set up multiple project alternatives for LCC analysis in a single input file when only a limited number of inputs are needed.

- $\quad$ DISCOUNT (version 3.9-07) is a stand-alone program that computes present-value, futurevalue, and annual-value factors for any discount rate and study period, including UPV* factors needed for federal LCC analyses of energy projects.

- $\quad$ ERATES (version 1.11) is a stand-alone program that generates block-rate, time-of-use-rate, and demand-rate schedules for electricity prices. It computes monthly and annual electricity costs that can be imported directly into BLCC4 and entered manually into BLCC5.

- $\quad$ EMISS (version 1.00) is a stand-alone program that generates files of local air-pollution emission coefficients for use with the BLCC4 program.

The set of LCC programs also includes

- $\quad$ EERC 1.0-07, Energy Escalation Rate Calculator, a program that computes an average rate of escalation for a specified time period, based on the DOE energy price escalation rates used for calculating the FEMP discount factors. This average rate can be used as an escalation rate for contract payments in Energy Savings Performance Contracts (ESPC) and Utility Energy Services Contracts (UESC).

The NIST BLCC programs provide comprehensive economic analysis capabilities for the evaluation of proposed capital investments that are expected to reduce the long-term operating costs of buildings and building systems. They compute the LCC for project alternatives, compare project alternatives in order to determine which has the lowest LCC, perform annual cash flow analysis, and compute net savings (NS), savings-to-investment ratio (SIR), and adjusted internal rate of return (AIRR) for project alternatives over their designated study period. The BLCC programs can be used to perform economic analysis of capital investment projects undertaken by federal, state, and local government agencies, as well as by the private sector (BLCC4). In their application to federal energy conservation and renewable energy projects, BLCC5 and BLCC4 are consistent with NIST Handbook 135, and the federal life-cycle cost methodology and procedures described in 10 CFR 436A and OMB Circular A-94. In their application to private-sector and non-federal public-sector projects, they are consistent with ASTM standards for building economics.

The U.S. Department of Energy was directed by legislation and executive order to make available to the private sector the methods, procedures, and related aids developed for federal use. In response to this directive, the National Institute of Standards and Technology, under sponsorship of the U.S. Department of Energy, published a life-cycle costing book for use by the private sector entitled Comprehensive Guide for Least-Cost Energy Decisions, NBS SP 709 (January 1987). The private sector guide is supported by the data provided here, as well as by the BLCC4 computer program. The BLCC4 program supersedes the NBSLCC program that is documented in SP 709.

The latest versions of the programs and publications described above can be downloaded from the DOE/FEMP web site at:

http://www.eere.energy.gov/femp/program/lifecycle.html

To request diskettes of BLCC4, a CD of BLCC5 and hard copies of the above publications, please call the Energy Efficiency and Renewable Energy (EERE) Information Center:

1-877-EERE-INF (1-877-337-3463) 
In some years, depending on funding availability, NIST, under the sponsorship of DOE, conducts three workshops:

The two-day Basic LCC Workshop explains the underlying theory of present-value analysis and integrates it with the FEMP criteria. The two-day Project-Oriented LCC Workshop builds on the Basic Workshop, focuses on the use of the BLCC computer programs and applies the LCC methodology to more complex issues. Further information is available from the EERE Information Center or the DOE/FEMP web site. The third workshop is a two-hour televised course that introduces the elements of life-cycle cost analysis of energy and water conservation projects.

FEMP-Qualified Instructors are available to conduct LCC workshops on their own account across the U.S. For a list of instructors e-mail blippiatt@nist.gov.

Three video training films in a series entitled "Least-Cost Energy Decisions for Buildings" have been prepared by NIST. These films include "Introduction to Life-Cycle Costing," "Uncertainty and Risk," and "Choosing Economic Evaluation Methods." The video films and companion workbooks can be ordered from

Video Transfer, Inc.

5800 Arundel Avenue

Rockville, MD 20852

301-881-0270

For further information on the Federal Energy Management Program, please visit http://www.eere.energy.gov/femp. 


\section{ACKNOWLEDGMENTS}

The authors wish to thank Cyrus Nasseri of the Federal Energy Management Program, U.S. Department of Energy (DOE), for his support and direction of this work. Appreciation is extended to Paul Kondis and Paul Holtberg, of the DOE Energy Information Administration, for providing the energy price projections upon which this report is based. Thanks are also due to Stephen Petersen and Sieglinde Fuller, who originated this publication, and to Tessa Beavers for her assistance in producing this document. 


\section{CONTENTS}

\section{Page}

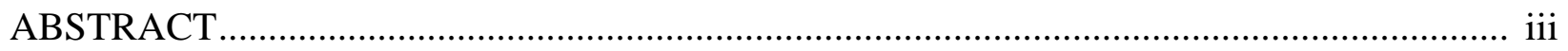

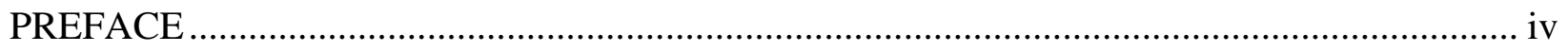

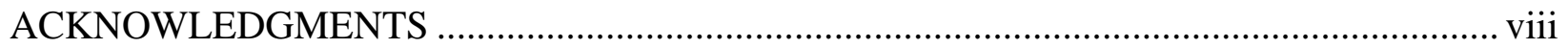

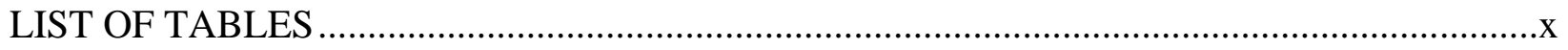

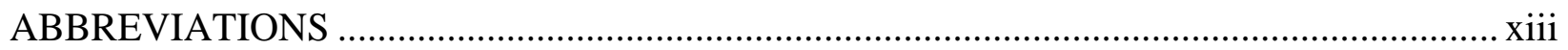

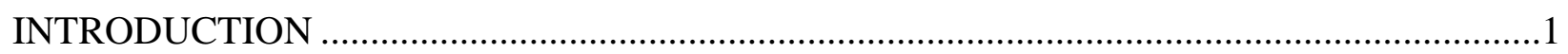

PART I: TABLES FOR FEDERAL LIFE-CYCLE COST ANALYSIS ..................................

A. Single Present Value and Uniform Present Value Factors for Non-Fuel Costs .................3

B. Modified Uniform Present Value Factors for Fuel Costs ............................................11

B.1. FEMP Modified Uniform Present Value Factors..................................................14

B.2. OMB Modified Uniform Present Value Factors ..................................................20

C. Projected Average Fuel Price Indices and Escalation Rates (Real)..............................26

PART II: ENERGY PRICE INDICES FOR PRIVATE SECTOR LCC ANALYSIS................43 


\section{LIST OF TABLES}

Page

A-1. $\quad$ SPV factors for finding the present value of future single costs (non-fuel) 6

A-2. $\quad$ UPV factors for finding the present value of future annually recurring uniform costs (non-fuel)

A-3a. $\quad \mathrm{UPV}^{*}$ factors for finding the present value of annually recurring costs changing at a constant escalation rate, DOE discount rate (3.0\%).

A-3b. $\quad \mathrm{UPV}^{*}$ factors for finding the present value of annually recurring costs changing at a constant escalation rate, OMB short-term discount rate $(2.7 \%)$.

A-3c. $\quad \mathrm{UPV}^{*}$ factors for finding the present value of annually recurring costs changing at a constant escalation rate, OMB long-term discount rate (3.0 \%) .............................10

Ba-1. $\quad$ FEMP UPV ${ }^{*}$ discount factors adjusted for fuel price escalation, by end-use sector and fuel type. Discount Rate = 3.0 \% (DOE). Census Region 1 (Connecticut, Maine, Massachusetts, New Hampshire, New Jersey, New York, Pennsylvania, Rhode Island, Vermont)

Ba-2. $\quad$ FEMP UPV ${ }^{*}$ discount factors adjusted for fuel price escalation, by end-use sector and fuel type. Discount Rate $=3.0$ \% (DOE). Census Region 2 (Illinois, Indiana, Iowa, Kansas, Michigan, Minnesota, Missouri, Nebraska, North Dakota, Ohio, South Dakota, Wisconsin)

Ba-3. $\quad$ FEMP UPV ${ }^{*}$ discount factors adjusted for fuel price escalation, by end-use sector and fuel type. Discount Rate $=3.0$ \% (DOE). Census Region 3 (Alabama, Arkansas, Delaware, District of Columbia, Florida, Georgia, Kentucky, Louisiana, Maryland, Mississippi, North Carolina, Oklahoma, South Carolina, Tennessee, Texas, Virginia, West Virginia).

Ba-4. $\quad$ FEMP UPV* discount factors adjusted for fuel price escalation, by end-use sector and fuel type. Discount Rate $=3.0$ \% (DOE). Census Region 4 (Alaska, Arizona, California, Colorado, Hawaii, Idaho, Montana, Nevada, New Mexico, Oregon, Utah, Washington, Wyoming) .18

Ba-5. $\quad$ FEMP UPV* discount factors adjusted for fuel price escalation, by end-use sector and fuel type. Discount Rate $=3.0 \%$ (DOE). United States Average .... 19

Bb-1. $\quad$ OMB UPV* discount factors adjusted for fuel price escalation, by end-use sector and fuel type. Discount Rate $=2.7 \%$ (years 1 to 10 ) and $3.0 \%$ (years 11 to 30), (OMB Circular A-94). Census Region 1 (Connecticut, Maine, Massachusetts, New Hampshire, New Jersey, New York, Pennsylvania, Rhode Island, Vermont) 


\section{LIST OF TABLES (continued)}

Bb-2. OMB UPV ${ }^{*}$ discount factors adjusted for fuel price escalation, by end-use sector and fuel type. Discount Rate $=2.7 \%$ (years 1 to 10 ) and $3.0 \%$ (years 11 to 30), (OMB Circular A-94). Census Region 2 (Illinois, Indiana, Iowa, Kansas, Michigan, Minnesota, Missouri, Nebraska, North Dakota, Ohio, South Dakota, Wisconsin) ....22

Bb-3. OMB UPV ${ }^{*}$ discount factors adjusted for fuel price escalation, by end-use sector and fuel type. Discount Rate $=2.7 \%$ (years 1 to 10 ) and $3.0 \%$ (years 11 to 30), (OMB Circular A-94). Census Region 3 (Alabama, Arkansas, Delaware, District of Columbia, Florida, Georgia, Kentucky, Louisiana, Maryland, Mississippi, North Carolina, Oklahoma, South Carolina, Tennessee, Texas, Virginia, West Virginia)...23

Bb-4. OMB UPV ${ }^{*}$ discount factors adjusted for fuel price escalation, by end-use sector and fuel type. Discount Rate $=2.7 \%$ (years 1 to 10 ) and $3.0 \%$ (years 11 to 30), (OMB Circular A-94). Census Region 4 (Alaska, Arizona, California, Colorado, Hawaii, Idaho, Montana, Nevada, New Mexico, Oregon, Utah, Washington, Wyoming)......24

Bb-5. OMB UPV ${ }^{*}$ discount factors adjusted for fuel price escalation, by end-use sector and fuel type. Discount Rate $=2.7 \%$ (years 1 to 10 ) and $3.0 \%$ (years 11 to 30), (OMB Circular A-94). United States Average.

Ca-1. Projected fuel price indices (excluding general inflation) by end-use sector and fuel type. Census Region 1 (Connecticut, Maine, Massachusetts, New Hampshire, New Jersey, New York, Pennsylvania, Rhode Island, Vermont).........................................28

Ca-2. Projected fuel price indices (excluding general inflation) by end-use sector and fuel type. Census Region 2 (Illinois, Indiana, Iowa, Kansas, Michigan, Minnesota, Missouri, Nebraska, North Dakota, Ohio, South Dakota, Wisconsin) ..........................30

Ca-3. Projected fuel price indices (excluding general inflation) by end-use sector and fuel type. Census Region 3 (Alabama, Arkansas, Delaware, District of Columbia, Florida, Georgia, Kentucky, Louisiana, Maryland, Mississippi, North Carolina, Oklahoma, South Carolina, Tennessee, Texas, Virginia, West Virginia) .......................................32

Ca-4. Projected fuel price indices (excluding general inflation) by end-use sector and fuel type. Census Region 4 (Alaska, Arizona, California, Colorado, Hawaii, Idaho, Montana, Nevada, New Mexico, Oregon, Utah, Washington, Wyoming) .................. 34

Ca-5. Projected fuel price indices (excluding general inflation) by end-use sector and fuel type. United States Average.

Cb-1. Projected average fuel price escalation rates (excluding general price inflation), by end-use sector and fuel type. Census Region 1 (Connecticut, Maine, Massachusetts, New Hampshire, New Jersey, New York, Pennsylvania, Rhode Island, Vermont) ...38 


\section{LIST OF TABLES (continued)}

Cb-2. Projected average fuel price escalation rates (excluding general price inflation), by end-use sector and fuel type. Census Region 2 (Illinois, Indiana, Iowa, Kansas, Michigan, Minnesota, Missouri, Nebraska, North Dakota, Ohio, South Dakota, Wisconsin)

Cb-3. Projected average fuel price escalation rates (excluding general price inflation), by end-use sector and fuel type. Census Region 3 (Alabama, Arkansas, Delaware, District of Columbia, Florida, Georgia, Kentucky, Louisiana, Maryland, Mississippi, North Carolina, Oklahoma, South Carolina, Tennessee, Texas, Virginia, West Virginia)

Cb-4. Projected average fuel price escalation rates (excluding general price inflation), by end-use sector and fuel type. Census Region 4 (Alaska, Arizona, California, Colorado, Hawaii, Idaho, Montana, Nevada, New Mexico, Oregon, Utah, Washington, Wyoming).

Cb-5. Projected average fuel price escalation rates (excluding general price inflation), by end-use sector and fuel type. United States Average.

S-1. Projected fuel price indices with assumed general price inflation rates of $2 \%$, $3 \%$, $4 \%$, and $5 \%$, by end-use sector and fuel type. Census Region 1 (Connecticut, Maine, Massachusetts, New Hampshire, New Jersey, New York, Pennsylvania, Rhode Island, Vermont)

S-2. Projected fuel price indices with assumed general price inflation rates of $2 \%, 3 \%$, $4 \%$, and $5 \%$, by end-use sector and fuel type. Census Region 2 (Illinois, Indiana, Iowa, Kansas, Michigan, Minnesota, Nebraska, North Dakota, Ohio, South Dakota, Wisconsin)

S-3. Projected fuel price indices with assumed general price inflation rates of $2 \%, 3 \%$, $4 \%$, and $5 \%$, by end-use sector and fuel type. Census Region 3 (Alabama, Arkansas, Delaware, District of Columbia, Florida, Georgia, Kentucky, Louisiana, Maryland, Mississippi, North Carolina, Oklahoma, South Carolina, Tennessee, Texas, Virginia, West Virginia).

S-4. $\quad$ Projected fuel price indices with assumed general price inflation rates of $2 \%, 3 \%$, $4 \%$, and $5 \%$, by end-use sector and fuel type. Census Region 4 (Alaska, Arizona, California, Colorado, Hawaii, Idaho, Montana, Nevada, New Mexico, Oregon, Utah, Washington, Wyoming)

S-5. Projected fuel price indices with assumed general price inflation rates of $2 \%, 3 \%$, $4 \%$, and $5 \%$, by end-use sector and fuel type. United States Average..............57 


\section{ABBREVIATIONS}

$\begin{array}{lll}\text { A } & - & \text { Annual amount } \\ \text { A }_{0} & - & \text { Annual amount at base-date prices } \\ \text { AEO2007 } & - & \text { Annual Energy Outlook } 2007 \text { (DOE-EIA publication) } \\ \text { ASTM } & - & \text { American Society for Testing and Materials } \\ \text { BLCC } & - & \text { NIST Building Life Cycle Cost computer program } \\ \text { COAL } & - & \text { Coal } \\ \text { d } & - & \text { discount rate } \\ \text { DIST } & - & \text { Distillate Oil } \\ \text { DOE } & - & \text { U.S. Department of Energy } \\ \text { e } & - & \text { price escalation rate (annual rate of price change) } \\ \text { EIA } & - & \text { Energy Information Administration (DOE) } \\ \text { ELEC } & - & \text { Electricity } \\ \text { ESPC } & - & \text { Energy Savings Performance Contract } \\ \text { FEMP } & - & \text { Federal Energy Management Program } \\ \text { FY } & - & \text { Fiscal Year } \\ \text { GASLN } & - & \text { Gasoline } \\ \text { LCC } & - & \text { Life-Cycle Cost } \\ \text { LPG } & - & \text { Liquefied petroleum gas } \\ \text { N } & - & \text { Number of discount periods (in years) } \\ \text { NEMS } & - & \text { National Energy Modeling System } \\ \text { NIST } & - & \text { National Institute of Standards and Technology } \\ \text { NTGAS } & - & \text { Natural Gas } \\ \text { OMB } & - & \text { Office of Management and Budget } \\ \text { RESID } & - & \text { Residual Oil } \\ \text { SPV } & - & \text { Single Present Value (factor) } \\ \text { UESC } & - & \text { Utility Energy Services Contract } \\ \text { UPV } & - & \text { Uniform Present Value (factor) } \\ \text { UPV } & - & \text { Modified Uniform Present Value (factor) }\end{array}$





\section{INTRODUCTION}

This report provides tables of present-value factors for use in the life-cycle cost analysis of capital investment projects for federal facilities. It also provides energy price indices based on Department of Energy (DOE) forecasts from 2007 to 2037. The factors and indices presented in this report are useful for determining the present value of future project-related costs, especially those related to operational energy costs. Discount factors included in this report are based on two different federal sources: (1) the DOE discount rate for projects related to energy conservation, renewable energy resources, and water conservation; and (2) Office of Management and Budget (OMB) discount rates from Circular A-94 for use with most other capital investment projects in federal facilities.

The DOE discount and inflation rates for 2007 are as follows:

$\begin{array}{ll}\text { Real rate (excluding general price inflation): } & 3.0 \% \\ \text { Nominal rate (including general price inflation): } & 5.0 \% \\ \text { Implied long-term average rate of inflation: } & 1.9 \%\end{array}$

The DOE nominal discount rate is based on long-term Treasury bond rates averaged over the 12 months prior to the preparation of this report. The nominal, or market, rate is converted to a real rate to correspond with the constant-dollar analysis approach used in most federal life-cycle cost (LCC) analyses. The method for calculating the real discount rate from the nominal discount rate is described in 10 CFR 436 and uses the projected rate of general inflation published in the most recent Report of the President's Economic Advisors, Analytical Perspectives. The procedure would result in a discount rate for 2007 lower than the $3.0 \%$ floor prescribed in 10 CFR 436. Thus the $3.0 \%$ floor is used as the real discount rate for FEMP analyses in 2007. The implied long-term average rate of inflation was calculated as $1.9 \%$. Federal agencies and contractors to federal agencies are required by 10 CFR 436 to use the DOE discount rates when conducting LCC analyses related to energy conservation, renewable energy resources, and water conservation projects for federal facilities.

The nominal and real discount rates applicable to general (non-energy or water) capital investments are published annually in OMB Circular A-94, Appendix C. OMB has specified two basic types of discount rates: (1) a discount rate for public investment and regulatory analyses; and (2) a discount rate for cost-effectiveness, lease-purchase, and related analyses. Only discount rates for the second type of analyses are included in this Annual Supplement, since the primary purpose of this report is to support cost-effectiveness studies related to the design and operation of federal facilities.

OMB discount rates for cost-effectiveness and lease-purchase studies are based on interest rates on Treasury Notes and Bonds with maturities ranging from 3 to 30 years. Currently (as of January 2007) five maturities have been specifically identified by OMB, and are shown here with the corresponding real interest rate to be used as the discount rate for studies subject to OMB Circular A-94:

\begin{tabular}{|c|c|c|c|c|}
\hline Maturity: & 3-year & 5-year & 7-year & 10-year \\
\hline Rate: & $2.5 \%$ & $2.6 \%$ & $2.7 \%$ & $2.8 \%$ \\
\hline
\end{tabular}

OMB suggests that the actual discount rate for an economic analysis be interpolated from these maturities and rates, based on the study period used in the analysis. Due to limitations on the size of this Annual Supplement, discount factors for only two of these maturities are presented: factors for short term analyses (up to 10 years) based on the 7-year real rate (2.7\%), and factors for long-term 
analyses (longer than 10 years) based on the 30-year real rate (3.0\%). As a result, these discount factors are for approximation purposes only. It is suggested that the NIST Building Life Cycle Cost (BLCC) or DISCOUNT programs be used to compute the present value factors for the discount rate corresponding to the length of the study period when approximate values are not satisfactory for the project analysis. (See preface for details on obtaining these programs.)

The energy price indices and corresponding present value factors published in this report are computed from energy price forecasts provided to NIST by the Department of Energy's Energy Information Administration (EIA). The EIA energy price forecast used in this report was the most recent available at the time that this report was prepared. A description of the methodology used by EIA to project energy prices through 2037 is included in section B of this report. DOE has not projected escalation rates for water prices to be used in the LCC analysis of water conservation projects. Water escalation rates should be obtained from the local water utility when possible.

Federal agencies and contractors to federal agencies are encouraged to seek energy price projections from their local utility to use in place of the DOE/EIA regional projections, especially when evaluating alternative fuel types. In such cases the NIST BLCC or DISCOUNT programs can be used to calculate appropriate "modified uniform present value" (UPV*) factors for use in the LCC analysis of federal energy conservation or renewable resource projects. Otherwise, 10 CFR 436 requires the use of the DOE energy price forecasts when conducting LCC analyses of such projects. The UPV* factors for energy costs presented in this report have been precalculated with the DOE forecast data. Thus the use of these UPV* factors automatically ensures that the DOE forecast data have been included in the analysis.

All of the tables of discount factors contained in this report are based on real discount rates and are therefore intended for use only with economic analyses conducted in constant dollars (in which the purchasing power of the dollar is held constant). The energy price escalation rates and corresponding energy price indices contained in this report are also expressed in real terms. If nominal discount rates and current dollar costs (which both include inflation) are used in the LCC analyses of federal projects, choose the current-dollar-analysis option in the BLCC5 computer program, which uses a nominal discount rate and adds the rate of general inflation to all dollar amounts.

This report uses the term "present value" instead of "present worth" for the discount factors presented. The meaning of these two terms is considered to be identical for purposes of economic analysis. This change in terminology was made to be consistent with the terms used in the American Society for Testing and Materials (ASTM) compilation of standards on building economics (ASTM Standards on Building Economics, Fifth Edition, ASTM, Philadelphia, PA, 2004.)

In all of the tables, the "end-of-year" discounting convention is used, that is, all factors and indices are computed to adjust future dollar amounts to present value from the end of the year in which they are expected to occur. The factors and indices in this publication, which include energy price escalation rates (e.g., UPV* factors and energy price indices), were calculated using April 1, 2007 (the date of this publication) as their base date. However, these factors and indices can be used without adjustment for the LCC analysis of projects with other base dates until the release of the next revision of this publication (April 2008). Adjustment of these factors and indices for differences in the month-specific base date is not generally warranted due to uncertainties in estimating future energy prices. 


\section{PART I: \\ TABLES FOR FEDERAL LIFE-CYCLE COST ANALYSIS}

\section{A. Single Present Value and Uniform Present Value Factors for Non-Fuel Costs}

Table A-1 presents the single present value (SPV) factors for finding the present value of future non-fuel, non-annually recurring costs, such as repair and replacement costs and salvage values. The formula for finding the present value $(P)$ of a future cost occurring in year $t\left(C_{t}\right)$ is the following:

$$
P=C_{t} \times \frac{1}{(1+d)^{t}}=C_{t} \times S P V_{t}
$$

where $\mathrm{d} \quad=$ discount rate, and

$\mathrm{t}=$ number of time periods (years) between the present time and the time the cost is incurred.

Table A-2 presents uniform present value (UPV) factors for finding the present value of future non-fuel costs recurring annually, such as routine maintenance costs. The formula for finding the present value $(\mathrm{P})$ of an annually recurring uniform cost $(\mathrm{A})$ is the following:

$$
P=A \times \frac{(1+d)^{N}-1}{d(1+d)^{N}}=A \times U P V_{N}
$$

where $\mathrm{d} \quad=$ discount rate, and

$\mathrm{N} \quad=$ number of time periods (years) over which A recurs.

Tables A-3 $(\boldsymbol{a}, \boldsymbol{b}, \boldsymbol{c})$ present modified uniform present value (UPV*) factors for finding the present value of annually recurring non-fuel costs, such as water costs, which are expected to change from year to year at a constant rate of change (or escalation rate) over the study period. The escalation rate can be positive or negative. The formula for finding the present value $(\mathrm{P})$ of an annually recurring cost at base-date prices $\left(\mathrm{A}_{0}\right)$ changing at escalation rate $\mathrm{e}$ is the following:

$$
P=A_{0} \times\left(\frac{1+e}{d-e}\right)\left[1-\left(\frac{1+e}{1+d}\right)^{N}\right]=A \times U P V^{*} \quad(d \neq e)
$$

or

$$
P=A_{0} \times N=A \times U P V^{*}{ }_{N} \quad(d=e),
$$

where $\mathrm{A}_{0} \quad=$ annually recurring cost at base-date prices,

$\mathrm{d}=$ discount rate,

e = escalation rate, and

$\mathrm{N} \quad=$ number of time periods (years) over which A recurs. 
Note: if the discount rate is expressed in real terms, i.e., net of general inflation, then the escalation rate must also be expressed in real terms. If the discount rate is expressed in nominal terms, i.e., including general inflation, then the escalation rate must also be expressed in nominal terms.

In tables A-1, A-2, and A-3 (a,b,c) SPV, UPV, and UPV* factors are provided for both the DOE and the OMB Circular A-94 real discount rates current as of the date of this publication. The FEMP SPV, UPV, and UPV* factors were computed using the DOE discount rate. The FEMP factors are for finding the present value of future costs associated with federal energy and water conservation projects and renewable energy projects. The OMB SPV, UPV, and UPV* factors were computed using the OMB discount rates. The OMB factors are for finding the present value of future costs associated with most other federal projects (except those specifically exempted from OMB Circular A-94). The DOE and OMB discount rates used in computing these tables are real rates, exclusive of general price inflation. Thus the resulting discount factors are intended for use with future costs that are stated in constant dollars.

Note: We have added to table A-3a a column of UPV* factors that incorporate an escalation rate of $-1.9 \%$, the negative of the inflation rate used to calculate the DOE nominal discount rate for 2007. The UPV* factors in this column can be used to calculate present values of fixed dollar amounts when performing a constant-dollar analysis. An example might be a fixed contract payment in an ESPC project. For these fixed amounts, the assumption that in a constant-dollar analysis all cash flows change at the rate of general inflation (so that the differential escalation rate is zero) does not apply. In real terms, fixed amounts change at a differential rate equal to the negative of the inflation rate.

\section{Examples of How to Use the Factors:}

SPV (FEMP): To compute the present value of a replacement cost expected to occur in the 8th year for an energy efficient heating system, go to Table A-1, find the 3.0 \% SPV factor for year 8 (0.789), and multiply the factor by the replacement cost as of the base date.

SPV (OMB, Short-term): To compute the present value of a repair cost in the 5th year for a floor covering (non-energy related), go to Table A-1, find the $2.7 \%$ SPV factor for year 5 (0.875), and multiply the factor by the repair cost as of the base date.

SPV (OMB, Long-term): To compute the present value of a repair cost in the 15th year for a floor covering (non-energy related), go to Table A-1, find the $3.0 \%$ SPV factor for year 15 (0.642), and multiply the factor by the repair cost as of the base date.

UPV (FEMP): To compute the present value of an annually recurring maintenance cost for a renewable energy system over 20 years, go to Table A-2, find the $3.0 \%$ UPV factor for 20 years (14.88), and multiply the factor by the annual maintenance cost as of the base date.

UPV (OMB, Short-term): To compute the present value of annually recurring costs of office cleaning over 10 years (for a project not primarily related to energy conservation), go to Table A-2, find the $2.7 \%$ UPV factor for 10 years (8.66), and multiply the factor by the annual cleaning cost as of the base date. 
UPV (OMB, Long-term): To compute the present value of annually recurring costs of office cleaning over 25 years (for a project not primarily related to energy conservation), got to Table A-2, find the $3.0 \%$ UPV factor for 25 years (17.41), and multiply the factor by the annual cleaning cost as of the base date.

$\mathrm{UPV}^{*}$ (all): To compute the present value of annually recurring costs of water usage which are expected to increase at $2 \%$ faster than the rate of general inflation over 25 years, find the UPV* factor from table A-3 (a ,b, or c as appropriate) that corresponds to $2 \%$ escalation and a 25 year study period. From table A-3a (3.0 \% DOE discount rate) the corresponding UPV* factor is 22.08. Multiply this factor by the annual water cost as computed at base year prices to determine the present value of these water costs over the entire 25 years.

$\mathrm{UPV}^{*}$ (negative inflation rate): To compute the present value of an annually recurring contract payment that is fixed over a contract period of 10 years, find the UPV* factor from table A-3a that corresponds to an escalation of $-1.9 \%$ and a 10-year time period. From table A-3a (3.0 \% DOE discount rate) the corresponding UPV* factor is 7.72. Multiply this factor by the annual contract payment as of the base year to determine the present value of these contract payments over the entire 10-year period.

Note: UPV factors are generally applied to costs that recur annually in substantially the same amount. Examples of such costs are routine operating and maintenance costs. UPV* factors are generally applied to costs that recur annually but change from year to year at a constant escalation rate. Examples of such costs are water usage costs when they increase from year to year. These costs usually occur every year over the service period of the building life. If there is a planning/design/construction period before the service life begins, during which these annual costs are not incurred, the appropriate UPV (or UPV*) factor for the service period is the difference between the UPV (or UPV*) factor for the entire study period and the UPV (or UPV*) factor for the planning/design/construction period. For example, if the planning/ design/construction period is 3 years and the service period is 25 years, for a total study period of 28 years, the corresponding UPV factor (from Table A-2, DOE $3.0 \%$ discount rate) is 18.76 $2.83=15.93$.

For further explanation and illustration of how to use these factors, see NIST Handbook 135. 
Table A-1. SPV factors for finding the present value of future single costs (non-fuel)

\begin{tabular}{|c|c|c|c|}
\hline-------- & \multicolumn{3}{|c|}{ Single Present Value (SPV) Factors } \\
\hline Number of & DOE & OMB Disc & unt Rates ${ }^{a}$ \\
\hline years from & Discount rate & Short term ${ }^{b}$ & Long Term $^{\mathrm{c}}$ \\
\hline base date & $3 . \odot \%$ & $2.7 \%$ & $3.0 \%$ \\
\hline$--_{-}-----$ & 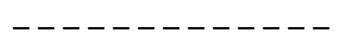 & -------- & ------- \\
\hline 0.25 & 0.993 & $\odot .993$ & 0.993 \\
\hline 0.50 & 0.985 & 0.987 & 0.985 \\
\hline 0.75 & 0.978 & 0.980 & 0.978 \\
\hline 1 & $\odot .971$ & 0.974 & 0.971 \\
\hline 2 & 0.943 & 0.948 & 0.943 \\
\hline 3 & 0.915 & 0.923 & $\odot .915$ \\
\hline 4 & 0.888 & $\odot .899$ & $\odot .888$ \\
\hline 5 & 0.863 & 0.875 & 0.863 \\
\hline 6 & 0.837 & 0.852 & 0.837 \\
\hline 7 & 0.813 & 0.830 & 0.813 \\
\hline 8 & 0.789 & 0.808 & 0.789 \\
\hline 9 & 0.766 & 0.787 & 0.766 \\
\hline 10 & 0.744 & 0.766 & 0.744 \\
\hline 11 & 0.722 & & 0.722 \\
\hline 12 & 0.701 & & 0.701 \\
\hline 13 & $\odot .681$ & & $\odot .681$ \\
\hline 14 & 0.661 & & 0.661 \\
\hline 15 & 0.642 & & 0.642 \\
\hline 16 & 0.623 & & 0.623 \\
\hline 17 & 0.605 & & 0.605 \\
\hline 18 & 0.587 & & 0.587 \\
\hline 19 & 0.570 & & 0.570 \\
\hline 20 & 0.554 & & 0.554 \\
\hline 21 & 0.538 & & 0.538 \\
\hline 22 & 0.522 & & 0.522 \\
\hline 23 & 0.507 & & 0.507 \\
\hline 24 & 0.492 & & 0.492 \\
\hline 25 & 0.478 & & 0.478 \\
\hline 26 & 0.464 & & 0.464 \\
\hline 27 & 0.450 & & 0.450 \\
\hline 28 & 0.437 & & 0.437 \\
\hline 29 & 0.424 & & 0.424 \\
\hline 30 & 0.412 & & 0.412 \\
\hline
\end{tabular}

${ }^{\mathrm{a}} \mathrm{OMB}$ discount rates as of February 2007.

${ }^{\text {b}}$ Short-term discount rate based on OMB discount rate for 7-year study period.

${ }^{\mathrm{c}}$ Long-term discount rate based on OMB discount rate for 30-year study period. 
Table A-2. UPV factors for finding the present value of annually recurring uniform costs (non-fuel)

\begin{tabular}{|c|c|c|c|}
\hline & \multicolumn{3}{|c|}{ Uniform Present Value (UPV) Factors } \\
\hline $\begin{array}{l}------ \\
\text { Number of }\end{array}$ & $\begin{array}{c}-\cdots \\
\text { DOE }\end{array}$ & OMB Disc & nt Rates \\
\hline years from & Discount rate & Short term & Long Term ${ }^{\mathrm{C}}$ \\
\hline base date & $3 . \odot \%$ & $2.7 \%$ & $3.0 \%$ \\
\hline-------- & 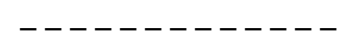 & 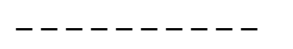 & 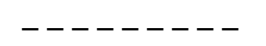 \\
\hline 1 & 0.97 & 0.97 & 0.97 \\
\hline 2 & 1.91 & 1.92 & 1.91 \\
\hline 3 & 2.83 & 2.85 & 2.83 \\
\hline 4 & 3.72 & 3.74 & 3.72 \\
\hline 5 & 4.58 & 4.62 & 4.58 \\
\hline 6 & 5.42 & 5.47 & 5.42 \\
\hline 7 & 6.23 & 6.30 & 6.23 \\
\hline 8 & 7.02 & 7.11 & 7.02 \\
\hline 9 & 7.79 & 7.90 & 7.79 \\
\hline 10 & 8.53 & 8.66 & 8.53 \\
\hline 11 & 9.25 & & 9.25 \\
\hline 12 & 9.95 & & 9.95 \\
\hline 13 & 10.63 & & 10.63 \\
\hline 14 & 11.30 & & 11.30 \\
\hline 15 & 11.94 & & 11.94 \\
\hline 16 & 12.56 & & 12.56 \\
\hline 17 & 13.17 & & 13.17 \\
\hline 18 & 13.75 & & 13.75 \\
\hline 19 & 14.32 & & 14.32 \\
\hline 20 & 14.88 & & 14.88 \\
\hline 21 & 15.42 & & 15.42 \\
\hline 22 & 15.94 & & 15.94 \\
\hline 23 & 16.44 & & 16.44 \\
\hline 24 & 16.94 & & 16.94 \\
\hline 25 & 17.41 & & 17.41 \\
\hline 26 & 17.88 & & 17.88 \\
\hline 27 & 18.33 & & 18.33 \\
\hline 28 & 18.76 & & 18.76 \\
\hline 29 & 19.19 & & 19.19 \\
\hline 30 & 19.60 & & 19.60 \\
\hline - - - - - - - - - & ---------- & -------- & -------- \\
\hline
\end{tabular}

${ }^{\mathrm{a} O M B}$ discount rates as of February 2007.

bShort-term discount rate based on OMB discount rate for 7-year study period.

${ }^{c}$ Long-term discount rate based on OMB discount rate for 30-year study period. 
Table A-3a. UPV* factors for finding the present value of annually recurring costs changing at a constant escalation rate, DOE discount rate.

DOE discount rate $=3.0 \%$

Modified Uniform Present Value (UPV*) Factors (non-fuel)

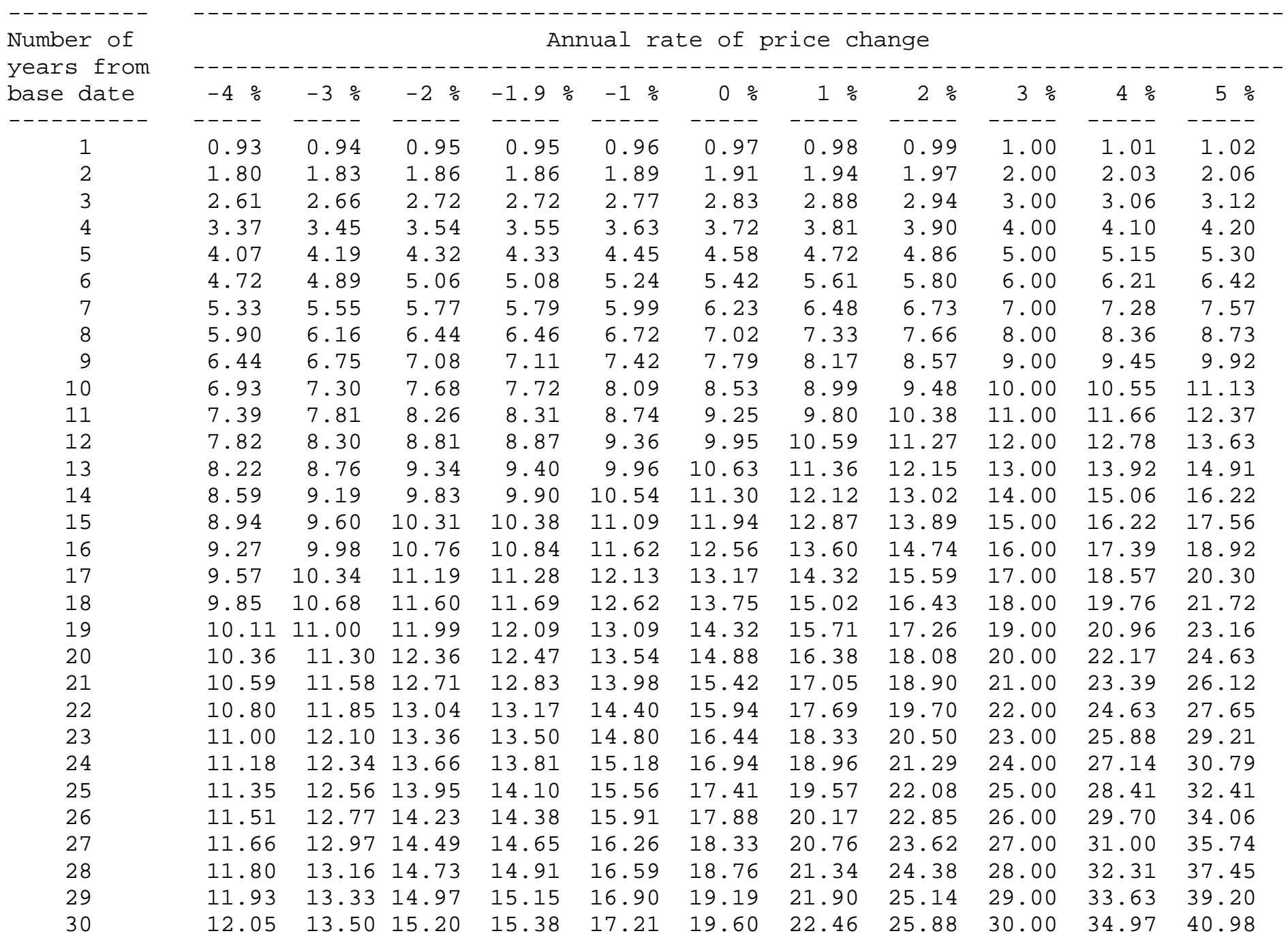


Table A-3b. UPV* factors for finding the present value of annually recurring amounts changing at a constant escalation rate, $\mathrm{OMB}$ short-term discount rate.

OMB short-term discount rate $=2.7 \%^{\mathrm{a}}$

Modified Uniform Present Value (UPV*) Factors (non-fuel)

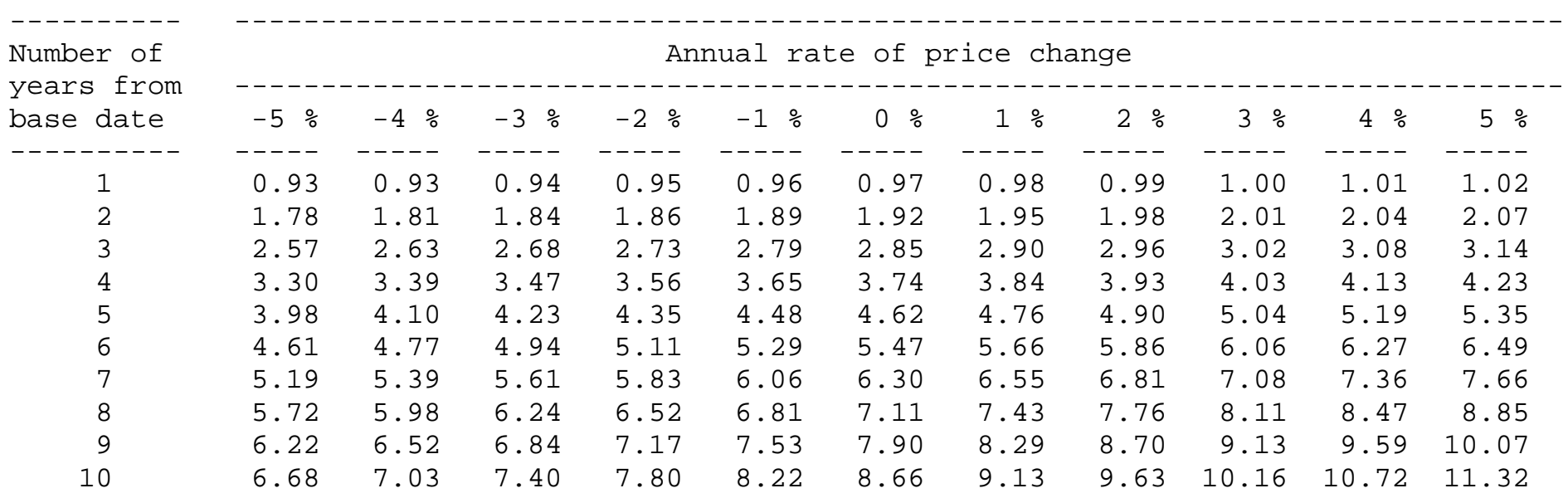

${ }^{\mathrm{a} O M B}$ discount rate as of February 2007.

Short-term discount rate based on OMB discount rate for 7-year study period. 
Table A-3c. UPV* factors for finding the present value of annually recurring amounts changing at a constant escalation rate, OMB long-term discount rate.

OMB long-term discount rate $=3.0 \%^{\mathrm{a}}$

Modified Uniform Present Value (UPV*) Factors (non-fuel)

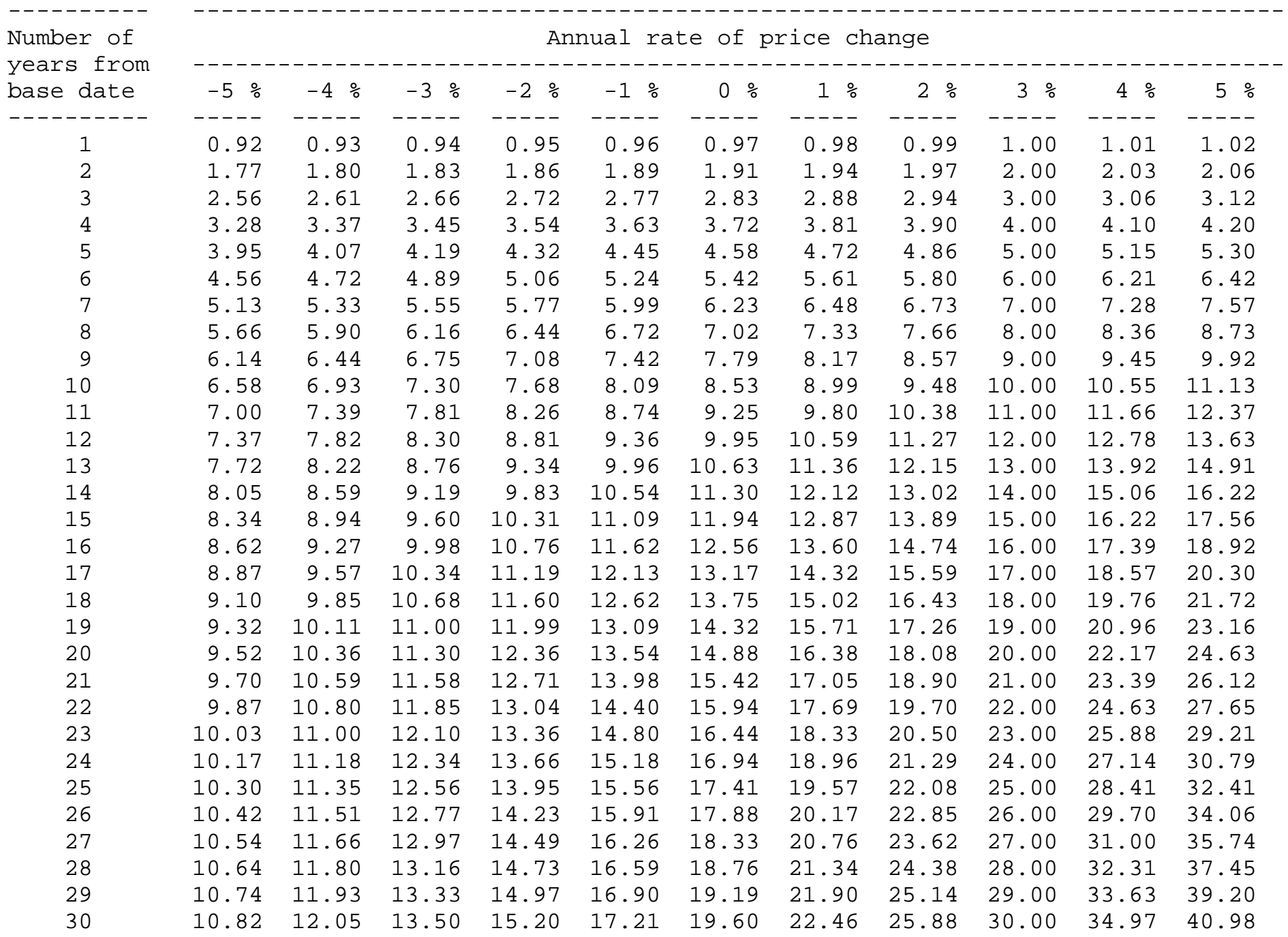

${ }^{\mathrm{a} O M B}$ discount rate as of February 2007.

Long-term discount rate based on OMB discount rate for 30-year study period. 


\section{B. Modified Uniform Present Value Factors for Fuel Costs}

This section presents FEMP and OMB modified uniform present value (UPV*) discount factors for calculating the present value of energy usage for federal projects. Factors are provided for the four major Census regions and for the overall United States. The factors are modified in the sense that they incorporate energy price escalation rates based on future energy prices projected by DOE for the years 2007 to 2037. There are two sets of UPV* tables: the "Ba" tables present FEMP UPV* factors based on the DOE discount rate $(3.0 \%$ real), and the "Bb" tables present OMB UPV* factors based on two OMB discount rates (2.7\% real for short-term study periods of 1 to 10 years, $3.0 \%$ real for long-term study periods of 11 to 30 years). The underlying energy price indices for the years 2007 to 2037, on which these UPV* calculations are based, are shown in tables Ca-1 through Ca-5. The corresponding average energy price escalation rates for selected time intervals between 2007 and 2037 are shown in tables Cb-1 through Cb-5.

\section{Energy Price Projections}

The FEMP and OMB UPV* factors incorporate energy price escalation rates computed from future energy prices projected by the Energy Information Administration (EIA) of the U.S. Department of Energy. Energy prices through 2030 were generated by EIA using the National Energy Modeling System (NEMS) and published in the Annual Energy Outlook 2007 (AEO2007). At the request of FEMP, EIA extrapolated energy prices from 2031 to 2037 based on selected growth rates from the AEO2007 projections.

NEMS is an energy market model designed to project the impacts of alternative energy policies or assumptions on U.S. energy markets. NEMS produces projections of the U.S. energy future, given current laws and policies and other key assumptions, including macroeconomic indicators from Data Resources, Inc., the production policy of the Organization of Petroleum Exporting Countries, the size of the economically recoverable resource base for fossil fuels, and the rate of development and penetration of new technologies. NEMS balances energy supply and demands with modules representing primary fuel supply, end-use demand for four sectors, and conversion of energy by refineries and electricity generators. Macroeconomic and international oil modules reflect the impacts of energy prices, production, and consumption on world oil markets and the economy.

The EIA energy price projections presented in this report, like those of other forecasters, are dependent on the data, methodologies, and specific assumptions used in their development. Many of the assumptions concerning the future cannot be known with any degree of certainty. Thus, the projections are not statements of what will happen, but what might happen given the particular assumptions and methodologies used. Although EIA has endeavored to make these forecasts as objective, reliable, and useful as possible, these projections should serve as an adjunct to, not a substitute for, the analytical process. The AEO2007 was prepared by EIA as required under statute by federal legislation. The price projections to 2037 were prepared in accordance with a Service Request from the Federal Energy Management Program. 


\section{UPV* Calculation Method}

The formula for finding the present value (P) of future energy costs or savings is the following:

$$
P=A_{0} x \sum_{i=1}^{N} \frac{I_{(2007+t)}}{(1+d)^{t}}=A_{0} x U P V_{N}^{*}
$$

where $\mathrm{A}_{0} \quad=$ annual cost of energy as of the base date (April 1, 2007);

$\mathrm{t} \quad=$ index used to designate the year of energy usage;

$\mathrm{N} \quad=$ number of periods, e.g., years, over which energy costs or savings accrue;

$\mathrm{I}_{(2007+\mathrm{t})}=$ projected average fuel price index ${ }^{1}$ given in Tables Ca- 1 through Ca-5 for the year $2007+\mathrm{t}$ (where $\mathrm{I}_{2007}=1.00$ ); and

$\mathrm{d}=$ the real discount rate.

This formula is based on end-of-year energy prices and end-of-year discounting. Note that annual energy costs as of the base date of the LCC analysis ( $\mathrm{A}_{0}$, to be supplied by the analyst) should reflect the current energy price schedule as of that date, which may not be the same as the energy price itself on that date. ${ }^{2}$ That is, the annual energy cost should reflect summer-winter rate differences, time-of-use rates, block rates considerations, and demand charges (as appropriate) anticipated to be in effect that year. If energy and demand costs are calculated separately (as is sometimes done for electricity), the UPV* factor should be applied to both costs.

The data in the tables that follow are reported for the four Census regions and the U.S. average. Figure B-1 presents a map showing the states corresponding to the four Census regions. The Census regions do not include American Samoa, Canal Zone, Guam, Puerto Rico, Trust Territory of the Pacific Islands, or the Virgin Islands. Analysts of federal projects in these areas should use data that are "reasonable under the circumstances," and may refer to the tables with U.S. average data for guidance.

\footnotetext{
${ }^{1}$ For greater precision, the UPV* factors reported in the Ba and $\mathrm{Bb}$ tables were computed using the unrounded form of the indices given in Tables Ca-1 through Ca-5.

${ }^{2}$ While the UPV* factors provided in this publication were computed using energy price indices that correspond to energy prices as of April 1 in the current and future years, the analyst is encouraged to use for determining $A_{0}$ the energy prices prevailing as of the base date of the LCC analysis for the project evaluated.
} 


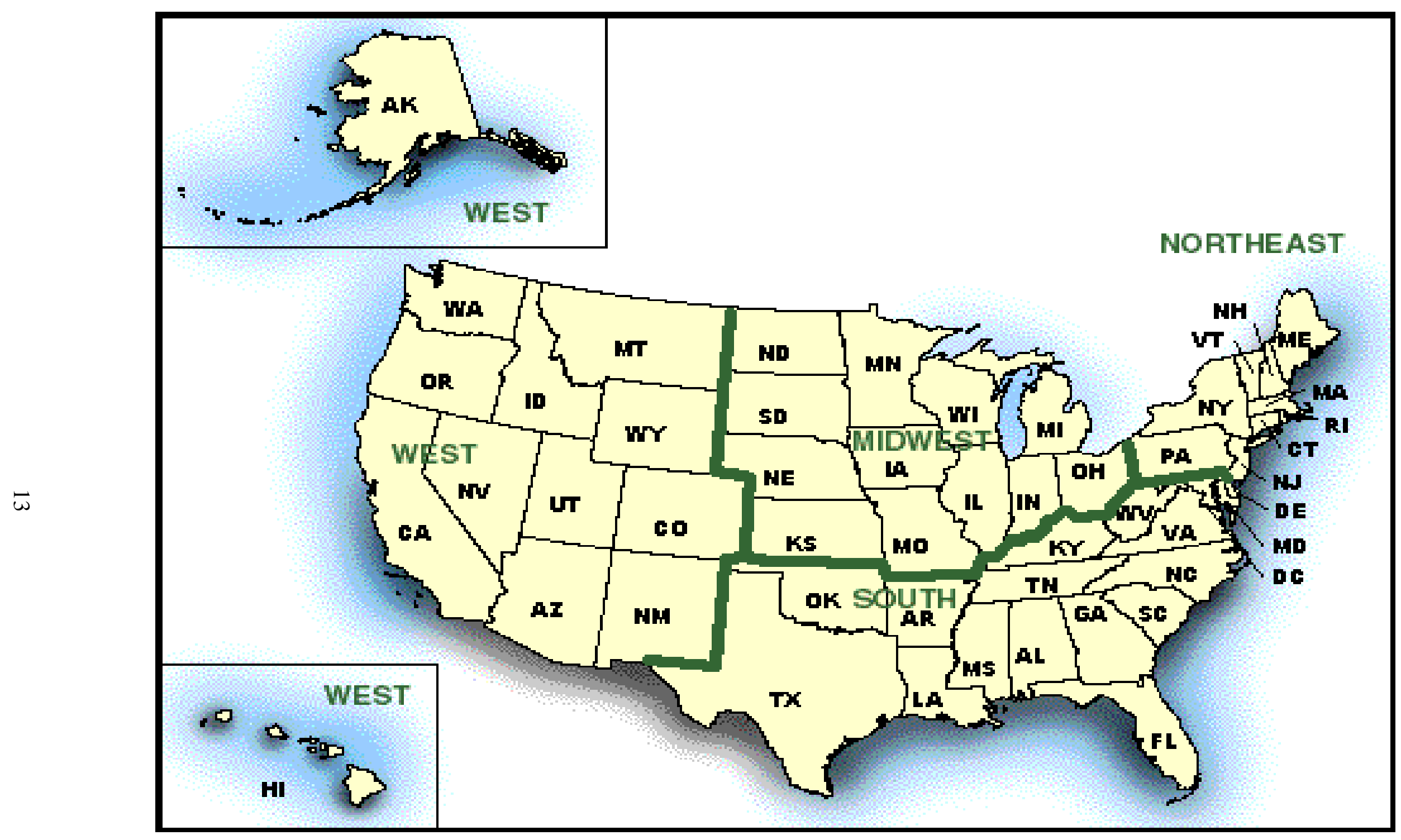

Figure B-1

Source: U.S. Census Bureau 


\section{B.1. FEMP Modified Uniform Present Value Factors}

The FEMP Modified Uniform Present Value (FEMP UPV*) factors presented in the "Ba" tables, based on the current DOE discount rate (3.0\%), are for calculating the present value of energy costs or savings accruing over 1 to 25 years and are to be used in life-cycle cost analyses of federal energy conservation and renewable energy projects. Factors are reported in the "Ba" tables for 30 years to accommodate a planning/design/construction period of up to 5 years. (See "Examples of How to Use FEMP UPV* Factors" below for instructions on use with planning/design/construction periods.)

These factors apply only to annual energy usage or energy savings that are assumed to be the same each year over the service period. The NIST BLCC computer program can compute the present value of energy usage and savings that are not the same in each year.

\section{Examples of How to Use the FEMP UPV* Factors:}

FEMP UPV*, no planning/design/construction period: To compute the present value of heating with natural gas over 25 years in a federal office building in New Mexico, go to Table Ba-4, find the FEMP UPV* factor for commercial natural gas for 25 years (15.03), and multiply this factor by the annual heating cost at base-date natural gas prices.

FEMP UPV*, with planning/design/construction period: To compute a present value factor for a service period following a planning/design/construction period (1) find the FEMP UPV* factor for the combined length of the planning/design/construction period and the service period (not to exceed 30 years), and (2) subtract from (1) the FEMP UPV* factor for the planning/design/ construction period alone. The difference is the FEMP UPV* factor for the years over which energy costs or savings actually accrue. For example, suppose a new federal office building in New York is being evaluated with several energy conserving design options. It is expected to have a planning/design/construction period of 5 years, after which it will be occupied for 25 years. To compute the present value of natural gas costs over 25 years of occupancy, go to Table Ba-1 and find the FEMP UPV* factors for commercial natural gas for 5 years (4.08) and for 30 years (16.66). The difference (12.58) is the FEMP UPV* factor for natural gas costs over 25 years, beginning 5 years after the base date. Multiply 12.58 by the annual natural gas cost at base date prices (not occupancy-date prices) to calculate the present value of natural gas costs over the entire 25-year occupancy period. 
Table Ba-1. FEMP UPV* Discount Factors adjusted for fuel price escalation, by end-use sector and fuel type.

\author{
Discount rate $=3.0 \%(\mathrm{DOE})$
}

Census Region 1 (Connecticut, Maine, Massachusetts, New Hampshire,

New Jersey, New York, Pennsylvania, Rhode Island, Vermont)

\begin{tabular}{rrrr}
\multicolumn{4}{c}{ RESIDENTIAL } \\
Elec & \multicolumn{1}{c}{ Dist } & \multicolumn{1}{l}{ LPG } & NtGas \\
.---- &.--- &..-- &..--- \\
0.95 & 0.96 & 1.00 & 0.94 \\
1.88 & 1.83 & 1.95 & 1.83 \\
2.76 & 2.63 & 2.86 & 2.66 \\
3.61 & 3.37 & 3.73 & 3.45 \\
4.44 & 4.04 & 4.56 & 4.20 \\
5.25 & 4.67 & 5.36 & 4.92 \\
6.03 & 5.26 & 6.14 & 5.61 \\
6.79 & 5.84 & 6.90 & 6.29 \\
7.53 & 6.40 & 7.63 & 6.94 \\
8.25 & 6.95 & 8.35 & 7.59 \\
8.96 & 7.49 & 9.04 & 8.22 \\
9.64 & 8.01 & 9.72 & 8.83 \\
10.31 & 8.53 & 10.37 & 9.42 \\
10.96 & 9.04 & 11.01 & 10.00 \\
11.59 & 9.54 & 11.63 & 10.57 \\
12.20 & 10.02 & 12.23 & 11.12 \\
12.80 & 10.50 & 12.82 & 11.67 \\
13.38 & 10.96 & 13.39 & 12.20 \\
13.95 & 11.41 & 13.95 & 12.72 \\
14.50 & 11.85 & 14.49 & 13.22 \\
15.03 & 12.28 & 15.01 & 13.72 \\
15.56 & 12.71 & 15.53 & 14.20 \\
16.07 & 13.12 & 16.03 & 14.68 \\
16.56 & 13.52 & 16.51 & 15.14 \\
17.04 & 13.91 & 16.98 & 15.60 \\
17.51 & 14.29 & 17.44 & 16.04 \\
17.96 & 14.66 & 17.88 & 16.47 \\
18.40 & 15.02 & 18.32 & 16.89 \\
18.83 & 15.37 & 18.74 & 17.30 \\
19.25 & 15.71 & 19.14 & 17.71
\end{tabular}

\begin{tabular}{rrrrr}
\multicolumn{5}{c}{ COMMERCIAL } \\
Elec & \multicolumn{1}{c}{ Dist } & Resid & NtGas & Coal \\
.---- &.--- &.---- &.--- &.--- \\
0.94 & 0.94 & 0.89 & 0.93 & 0.97 \\
1.83 & 1.80 & 1.74 & 1.80 & 1.90 \\
2.66 & 2.58 & 2.55 & 2.61 & 2.80 \\
3.43 & 3.30 & 3.31 & 3.37 & 3.66 \\
4.18 & 3.96 & 4.02 & 4.08 & 4.47 \\
4.90 & 4.57 & 4.62 & 4.76 & 5.23 \\
5.60 & 5.16 & 5.22 & 5.42 & 5.96 \\
6.28 & 5.72 & 5.82 & 6.05 & 6.66 \\
6.95 & 6.28 & 6.40 & 6.66 & 7.33 \\
7.60 & 6.82 & 6.97 & 7.27 & 7.97 \\
8.24 & 7.35 & 7.54 & 7.86 & 8.59 \\
8.86 & 7.88 & 8.12 & 8.43 & 9.18 \\
9.47 & 8.40 & 8.69 & 8.98 & 9.76 \\
10.05 & 8.90 & 9.25 & 9.52 & 10.33 \\
10.62 & 9.40 & 9.81 & 10.05 & 10.88 \\
11.18 & 9.88 & 10.35 & 10.56 & 11.43 \\
11.72 & 10.35 & 10.88 & 11.07 & 11.96 \\
12.24 & 10.82 & 11.38 & 11.56 & 12.48 \\
12.75 & 11.27 & 11.87 & 12.04 & 12.99 \\
13.24 & 11.71 & 12.35 & 12.50 & 13.48 \\
13.73 & 12.15 & 12.83 & 12.96 & 13.97 \\
14.20 & 12.58 & 13.27 & 13.41 & 14.45 \\
14.65 & 13.00 & 13.71 & 13.85 & 14.92 \\
15.09 & 13.41 & 14.13 & 14.28 & 15.38 \\
15.52 & 13.81 & 14.54 & 14.69 & 15.83 \\
15.93 & 14.20 & 14.94 & 15.10 & 16.26 \\
16.33 & 14.57 & 15.33 & 15.50 & 16.69 \\
16.72 & 14.94 & 15.71 & 15.90 & 17.10 \\
17.10 & 15.29 & 16.08 & 16.28 & 17.50 \\
17.47 & 15.64 & 16.44 & 16.66 & 17.90
\end{tabular}

\begin{tabular}{|c|c|c|c|c|}
\hline \multirow[b]{2}{*}{ Elec } & \multicolumn{4}{|c|}{ INDUSTRIAL } \\
\hline & Dist & Resid & NtGas & Coal \\
\hline & $-\cdots$ & $-\cdots$ & & \\
\hline 0.96 & 0.93 & 0.92 & 0.95 & 0.98 \\
\hline 1.86 & 1.77 & 1.81 & 1.81 & 1.93 \\
\hline 2.66 & 2.56 & 2.67 & 2.60 & 2.87 \\
\hline 3.39 & 3.29 & 3.46 & 3.34 & 3.78 \\
\hline 4.07 & 3.97 & 4.20 & 4.02 & 4.64 \\
\hline 4.71 & 4.60 & 4.85 & 4.66 & 5.47 \\
\hline 5.33 & 5.21 & 5.49 & 5.28 & 6.26 \\
\hline 5.92 & 5.80 & 6.12 & 5.88 & 7.02 \\
\hline 6.50 & 6.38 & 6.74 & 6.46 & 7.75 \\
\hline 7.07 & 6.95 & 7.35 & 7.04 & 8.45 \\
\hline 7.64 & 7.50 & 7.95 & 7.60 & 9.12 \\
\hline 8.18 & 8.05 & 8.56 & 8.14 & 9.76 \\
\hline 8.71 & 8.59 & 9.16 & 8.67 & 10.39 \\
\hline 9.23 & 9.12 & 9.75 & 9.19 & 11.01 \\
\hline 9.73 & 9.64 & 10.34 & 9.69 & 11.61 \\
\hline 10.22 & 10.14 & 10.90 & 10.18 & 12.19 \\
\hline 10.70 & 10.63 & 11.45 & 10.67 & 12.76 \\
\hline 11.17 & 11.11 & 11.98 & 11.14 & 13.32 \\
\hline 11.62 & 11.59 & 12.49 & 11.61 & 13.86 \\
\hline 12.06 & 12.05 & 13.00 & 12.06 & 14.39 \\
\hline 12.49 & 12.51 & 13.49 & 12.51 & 14.91 \\
\hline 12.91 & 12.96 & 13.96 & 12.95 & 15.42 \\
\hline 13.31 & 13.40 & 14.42 & 13.37 & 15.92 \\
\hline 13.70 & 13.83 & 14.86 & 13.79 & 16.40 \\
\hline 14.09 & 14.25 & 15.29 & 14.21 & 16.88 \\
\hline 14.46 & 14.65 & 15.71 & 14.61 & 17.34 \\
\hline 14.82 & 15.05 & 16.12 & 15.01 & 17.78 \\
\hline 15.17 & 15.43 & 16.52 & 15.40 & 18.22 \\
\hline 15.52 & 15.80 & 16.90 & 15.78 & 18.65 \\
\hline 15.85 & 16.17 & 17.28 & 16.15 & 19.06 \\
\hline
\end{tabular}

TRANSPORT

Gasln

0.92

1.76

2.53

3.26

3.95

4.60
5.22

5.82

6.40

6.96

7.52

8.07

9.12

9.63

10.13

10.62

11.09

11.55

11.55

12.00

12.44
12.87

12.87
13.29

13.70

14.10

14.49

14.86

15.23

15.58

15.93

Note: UPV* factors are reported for years 26-30 to accommodate a planning/construction period of up to 5 years.

See p. 14 for instructions on use; page xiii for abbreviations. 
Table Ba-2. FEMP UPV* Discount Factors adjusted for fuel price escalation, by end-use sector and fuel type.

Discount Rate $=3 . \odot \%($ DOE $)$

Census Region 2 (Illinois, Indiana, Iowa, Kansas, Michigan, Minnesota,

Missouri, Nebraska, North Dakota, Ohio, South Dakota, Wisconsin)

\begin{tabular}{|c|c|c|c|}
\hline \multicolumn{4}{|c|}{ RESIDENTIAL } \\
\hline Lec & Dist & LPG & NtGas \\
\hline & & & \\
\hline .98 & 0.95 & 1.02 & $0 . \varsigma$ \\
\hline & 1.82 & 1.99 & 1.8 \\
\hline .87 & & & \\
\hline .78 & 3.32 & 3.80 & 3.42 \\
\hline .66 & 3.97 & 4.65 & 4.16 \\
\hline .50 & 4.55 & 5.47 & 4.86 \\
\hline .32 & 5. & 6.27 & \\
\hline 7.11 & & 7. & \\
\hline 7.88 & 6.17 & 7.79 & .84 \\
\hline .63 &.$\epsilon$ & .52 & $.4 \varepsilon$ \\
\hline 9.36 & & 9.23 & 8 \\
\hline 10.07 & 7.68 & 9.92 & 8.7 \\
\hline .76 & 17 & .60 & 9.2 \\
\hline .44 & 8.64 & .25 & 9.8 \\
\hline .09 & 9.11 & 1.89 & 10.4 \\
\hline .7 & 7 & 2.51 & 0.9 \\
\hline .35 & 10.01 & 3.11 & 1.5 \\
\hline .95 & 10.45 & 13.70 & 12.0 \\
\hline .53 & & 4.28 & \\
\hline .09 & 11.28 & 14.83 & 13.0 \\
\hline 5.64 & 11.6 & 15.38 & 13.5 \\
\hline .17 & & 15.91 & \\
\hline .68 & 12. & 16.43 & \\
\hline .19 & 12.85 & 16.93 & 14.9 \\
\hline 7.67 & & 17.42 & 15.4 \\
\hline 15 & & 17.89 & \\
\hline 18 & & 18.35 & \\
\hline 19.06 & 14. & 18.80 & \\
\hline & & & \\
\hline & 140 & 1960 & \\
\hline
\end{tabular}

\begin{tabular}{|c|c|c|c|c|}
\hline \multicolumn{5}{|c|}{ COMMERCIAL } \\
\hline Elec & Dist & Resid & NtGas & Coal \\
\hline & & & & \\
\hline$\odot .98$ & 0.93 & 1.06 & 0.94 & $\odot .98$ \\
\hline 1.95 & 1.77 & 2.05 & 1.81 & 1.94 \\
\hline 2.89 & 2.55 & 2.97 & 2.63 & 2.90 \\
\hline 3.79 & 3.25 & 3.82 & 3.39 & 3.84 \\
\hline 4.65 & 3.89 & 4.61 & 4.11 & 4.76 \\
\hline 5.48 & 4.48 & 5.36 & 4.79 & 5.64 \\
\hline 6.27 & 5.04 & 6.08 & 5.44 & 6.50 \\
\hline 7.04 & 5.58 & 6.77 & 6.08 & 7.33 \\
\hline 7.79 & 6.11 & 7.44 & 6.70 & 8.12 \\
\hline 8.53 & 6.62 & 8.10 & 7.31 & 8.89 \\
\hline 9.26 & 7.13 & 8.74 & 7.91 & 9.63 \\
\hline 9.97 & 7.64 & 9.38 & 8.48 & 10.35 \\
\hline 10.66 & 8.13 & 10.00 & 9.05 & 11.05 \\
\hline 11.33 & 8.61 & 10.62 & 9.59 & 11.72 \\
\hline 11.99 & 9.09 & 11.22 & 10.13 & 12.38 \\
\hline 12.63 & 9.55 & 11.81 & 10.65 & 13.01 \\
\hline 13.26 & 10.00 & 12.38 & 11.17 & 13.64 \\
\hline 13.88 & 10.44 & 12.94 & 11.67 & 14.24 \\
\hline 14.47 & 10.87 & 13.48 & 12.16 & 14.84 \\
\hline 15.04 & 11.30 & 14.01 & 12.64 & 15.42 \\
\hline 15.59 & 11.72 & 14.53 & 13.11 & 15.98 \\
\hline 16.14 & 12.13 & 15.04 & 13.57 & 16.53 \\
\hline 16.66 & 12.53 & 15.54 & 14.02 & 17.07 \\
\hline 17.17 & 12.92 & 16.03 & 14.46 & 17.60 \\
\hline 17.67 & 13.30 & 16.50 & 14.89 & 18.11 \\
\hline 18.15 & 13.67 & 16.96 & 15.31 & 18.60 \\
\hline 18.62 & 14.03 & $17.4 \odot$ & 15.72 & 19.09 \\
\hline 19.08 & 14.38 & 17.84 & 16.13 & 19.56 \\
\hline 19.52 & 14.71 & 18.26 & 16.52 & 20.02 \\
\hline 19.96 & 15.04 & 18.67 & 16.91 & 20.48 \\
\hline
\end{tabular}

\begin{tabular}{|c|c|c|c|c|}
\hline \multirow[b]{2}{*}{ Elec } & \multicolumn{3}{|c|}{ INDUSTRIAL } & \multirow[b]{2}{*}{ Coal } \\
\hline & Dist & Resid & NtGas & \\
\hline 1.00 & 0.92 & 1.05 & 0.97 & 0.98 \\
\hline 1.98 & 1.75 & 2.04 & 1.86 & 1.93 \\
\hline 2.91 & 2.53 & 2.95 & 2.69 & 2.88 \\
\hline 3.78 & 3.26 & 3.80 & 3.46 & 3.81 \\
\hline 4.59 & 3.94 & 4.59 & 4.18 & 4.71 \\
\hline 5.37 & 4.58 & 5.33 & 4.86 & 5.58 \\
\hline 6.11 & 5.19 & 6.04 & 5.51 & 6.42 \\
\hline 6.83 & 5.77 & 6.73 & 6.14 & 7.23 \\
\hline 7.53 & 6.35 & 7.40 & 6.76 & 8.01 \\
\hline 8.22 & 6.92 & 8.06 & 7.38 & 8.77 \\
\hline 8.90 & 7.47 & 8.70 & 7.98 & 9.51 \\
\hline 9.57 & 8.02 & 9.33 & 8.56 & 10.23 \\
\hline 10.22 & 8.55 & 9.95 & 9.12 & 10.93 \\
\hline 10.86 & 9.08 & 10.56 & 9.67 & 11.61 \\
\hline 11.47 & 9.59 & 11.17 & 10.21 & 12.27 \\
\hline 12.08 & 10.09 & 11.75 & 10.74 & 12.92 \\
\hline 12.67 & 10.57 & 12.32 & 11.27 & 13.55 \\
\hline 13.25 & 11.05 & 12.87 & 11.78 & 14.16 \\
\hline 13.80 & 11.52 & 13.41 & 12.27 & 14.77 \\
\hline 14.34 & 11.98 & 13.94 & 12.76 & 15.35 \\
\hline 14.87 & 12.43 & 14.46 & 13.24 & 15.93 \\
\hline 15.38 & 12.87 & 14.97 & 13.71 & 16.49 \\
\hline 15.87 & 13.31 & 15.47 & 14.17 & 17.03 \\
\hline 16.35 & 13.73 & 15.95 & 14.63 & 17.57 \\
\hline 16.82 & 14.14 & 16.42 & 15.07 & 18.09 \\
\hline 17.28 & 14.54 & 16.87 & 15.51 & 18.59 \\
\hline 17.73 & 14.93 & 17.32 & 15.93 & 19.09 \\
\hline 18.16 & 15.31 & 17.75 & 16.35 & 19.57 \\
\hline 18.58 & 15.67 & 18.17 & 16.77 & 20.04 \\
\hline 18.99 & 16.03 & 18.58 & 17.17 & 20.50 \\
\hline
\end{tabular}

Gasln

0.93

1.78

2.57

3.30

3.99

4.65

5.27
5.87

6.45

7.02

7.57

8.12

8.66

9.18

9.69

10.19

10.68

11.16

11.62

12.07

12.51

12.94

13.36

13.78

14.18

14.57

14.94

15.31

15.67

$\begin{array}{lllllllll}19.91 & 14.90 & 19.66 & 17.57 & 19.96 & 15.04 & 18.67 & 16.91 & 20.48\end{array}$

$18.99 \quad 16.03 \quad 18.58 \quad 17.17 \quad 20.50$

Note: UPV* factors are reported for years 26-30 to accommodate a planning/construction period of up to 5 years.

See p. 14 for instructions on use; page xiii for abbreviations. 


\section{Table Ba-3. FEMP UPV* Discount Factors adjusted for fuel price escalation, by end-use sector and fuel type.}

Discount Rate $=3.0 \%(\mathrm{DOE})$

Census Region 3 (Alabama, Arkansas, Delaware, District of Columbia, Florida, Georgia, Kentucky, Louisiana, Maryland, Mississippi, North Carolina, Oklahoma, South Carolina, Tennessee, Texas, Virginia, West Virginia)

\begin{tabular}{rrrrr} 
& \multicolumn{4}{c}{ RESIDENTIAL } \\
$\mathrm{N}$ & \multicolumn{1}{c}{ Elec } & Dist & \multicolumn{1}{l}{ LPG } & NtGas \\
- &.--- &.--- &..-- &..-- \\
1 & 0.96 & 0.96 & 1.00 & 0.94 \\
2 & 1.89 & 1.84 & 1.95 & 1.83 \\
3 & 2.78 & 2.64 & 2.86 & 2.67 \\
4 & 3.63 & 3.37 & 3.74 & 3.45 \\
5 & 4.44 & 4.05 & 4.57 & 4.19 \\
6 & 5.23 & 4.67 & 5.38 & 4.91 \\
7 & 5.98 & 5.26 & 6.16 & 5.60 \\
8 & 6.71 & 5.84 & 6.92 & 6.27 \\
9 & 7.42 & 6.40 & 7.66 & 6.93 \\
10 & 8.12 & 6.95 & 8.38 & 7.58 \\
11 & 8.80 & 7.49 & 9.08 & 8.21 \\
12 & 9.46 & 8.01 & 9.76 & 8.83 \\
13 & 10.10 & 8.53 & 10.43 & 9.44 \\
14 & 10.72 & 9.04 & 11.07 & 10.02 \\
15 & 11.33 & 9.54 & 11.70 & 10.60 \\
16 & 11.91 & 10.02 & 12.31 & 11.16 \\
17 & 12.49 & 10.50 & 12.91 & 11.72 \\
18 & 13.04 & 10.96 & 13.49 & 12.26 \\
19 & 13.58 & 11.41 & 14.05 & 12.78 \\
20 & 14.11 & 11.85 & 14.60 & 13.30 \\
21 & 14.62 & 12.29 & 15.13 & 13.80 \\
22 & 15.11 & 12.71 & 15.65 & 14.30 \\
23 & 15.60 & 13.13 & 16.16 & 14.78 \\
24 & 16.06 & 13.53 & 16.65 & 15.25 \\
25 & 16.52 & 13.92 & 17.13 & 15.71 \\
26 & 16.96 & 14.30 & 17.59 & 16.16 \\
27 & 17.39 & 14.67 & 18.04 & 16.60 \\
28 & 17.81 & 15.03 & 18.48 & 17.03 \\
29 & 18.22 & 15.38 & 18.91 & 17.45 \\
30 & 18.62 & 15.72 & 19.32 & 17.86
\end{tabular}

\begin{tabular}{|c|c|c|c|c|}
\hline \multicolumn{5}{|c|}{ COMMERCIAL } \\
\hline Elec & Dist & Resid & NtGas & Coal \\
\hline & & & & \\
\hline 0.97 & 0.95 & 0.99 & 0.94 & 0.97 \\
\hline 1.90 & 1.80 & 1.90 & 1.82 & 1.92 \\
\hline 2.80 & 2.58 & 2.74 & 2.64 & 2.82 \\
\hline 3.66 & 3.29 & 3.50 & 3.39 & 3.68 \\
\hline 4.47 & 3.94 & 4.21 & 4.10 & 4.50 \\
\hline 5.26 & 4.54 & 4.87 & 4.78 & 5.28 \\
\hline 6.02 & 5.11 & 5.49 & 5.44 & 6.04 \\
\hline 6.76 & 5.67 & 6.10 & 6.08 & 6.78 \\
\hline 7.47 & 6.21 & 6.69 & 6.70 & 7.50 \\
\hline 8.18 & 6.74 & 7.27 & 7.31 & 8.19 \\
\hline 8.87 & 7.26 & 7.84 & 7.91 & 8.85 \\
\hline 9.54 & 7.78 & 8.40 & 8.49 & 9.48 \\
\hline 10.20 & 8.29 & 8.95 & 9.05 & 10.09 \\
\hline 10.83 & 8.78 & 9.50 & 9.60 & 10.68 \\
\hline 11.45 & 9.27 & 10.05 & 10.14 & 11.26 \\
\hline 12.05 & 9.74 & 10.57 & 10.67 & 11.82 \\
\hline 12.64 & 10.21 & 11.08 & 11.18 & 12.37 \\
\hline 13.21 & 10.66 & 11.59 & 11.69 & 12.90 \\
\hline 13.76 & 11.11 & 12.08 & 12.18 & 13.42 \\
\hline 14.30 & 11.54 & 12.56 & 12.66 & 13.92 \\
\hline 14.82 & 11.98 & 13.03 & 13.13 & 14.40 \\
\hline 15.33 & 12.40 & 13.50 & 13.59 & 14.88 \\
\hline 15.82 & 12.81 & 13.95 & 14.04 & 15.34 \\
\hline 16.30 & 13.22 & 14.40 & 14.47 & 15.80 \\
\hline 16.77 & 13.61 & 14.83 & 14.90 & 16.24 \\
\hline 17.22 & 13.99 & 15.25 & 15.32 & 16.67 \\
\hline 17.66 & 14.36 & 15.65 & 15.73 & 17.09 \\
\hline 18.09 & 14.72 & 16.05 & 16.13 & 17.50 \\
\hline 18.50 & 15.07 & 16.44 & 16.53 & 17.89 \\
\hline & $15 \quad 11$ & & & 1828 \\
\hline
\end{tabular}

\begin{tabular}{rrrrr}
\multicolumn{5}{c}{ INDUSTRIAL } \\
Elec & \multicolumn{1}{c}{ Dist } & Resid & NtGas & Coal \\
.---- &.--- &.---- &.--- &.--- \\
0.98 & 0.93 & 1.01 & 0.96 & 0.97 \\
1.91 & 1.77 & 1.95 & 1.82 & 1.91 \\
2.81 & 2.55 & 2.81 & 2.61 & 2.82 \\
3.65 & 3.28 & 3.61 & 3.32 & 3.69 \\
4.45 & 3.95 & 4.35 & 3.98 & 4.53 \\
5.21 & 4.59 & 5.04 & 4.60 & 5.33 \\
5.94 & 5.19 & 5.70 & 5.21 & 6.11 \\
6.65 & 5.78 & 6.34 & 5.79 & 6.86 \\
7.35 & 6.36 & 6.96 & 6.36 & 7.59 \\
8.03 & 6.92 & 7.57 & 6.93 & 8.30 \\
8.70 & 7.47 & 8.16 & 7.49 & 8.98 \\
9.36 & 8.02 & 8.75 & 8.04 & 9.64 \\
9.99 & 8.55 & 9.33 & 8.57 & 10.28 \\
10.61 & 9.08 & 9.90 & 9.09 & 10.90 \\
11.21 & 9.60 & 10.47 & 9.60 & 11.50 \\
11.80 & 10.10 & 11.01 & 10.10 & 12.09 \\
12.37 & 10.58 & 11.55 & 10.61 & 12.66 \\
12.93 & 11.07 & 12.07 & 11.09 & 13.21 \\
13.46 & 11.54 & 12.58 & 11.57 & 13.76 \\
13.99 & 12.00 & 13.09 & 12.03 & 14.28 \\
14.50 & 12.45 & 13.57 & 12.49 & 14.79 \\
14.99 & 12.90 & 14.06 & 12.94 & 15.29 \\
15.47 & 13.34 & 14.53 & 13.39 & 15.78 \\
15.94 & 13.77 & 14.98 & 13.82 & 16.26 \\
16.39 & 14.18 & 15.43 & 14.24 & 16.72 \\
16.83 & 14.58 & 15.86 & 14.66 & 17.17 \\
17.26 & 14.98 & 16.28 & 15.08 & 17.61 \\
17.68 & 15.36 & 16.69 & 15.48 & 18.04 \\
18.09 & 15.73 & 17.09 & 15.88 & 18.46 \\
18.48 & 16.09 & 17.47 & 16.27 & 18.86
\end{tabular}

$\begin{array}{rr}\text { TRANSPORT } & \\ \text { Gasln } & \mathrm{N} \\ ---- & - \\ 0.92 & 1 \\ 1.77 & 2 \\ 2.55 & 3 \\ 3.28 & 4 \\ 3.96 & 5 \\ 4.62 & 6 \\ 5.24 & 7 \\ 5.83 & 8 \\ 6.41 & 9 \\ 6.98 & 10 \\ 7.53 & 11 \\ 8.08 & 12 \\ 8.61 & 13 \\ 9.14 & 14 \\ 9.65 & 15 \\ 10.15 & 16 \\ 10.63 & 17 \\ 11.11 & 18 \\ 11.57 & 19 \\ 12.02 & 20 \\ 12.46 & 21 \\ 12.90 & 22 \\ 13.32 & 23 \\ 13.73 & 24 \\ 14.13 & 25 \\ 14.52 & 26 \\ 14.90 & 27 \\ 15.27 & 28 \\ 15.62 & 29 \\ 15.97 & 30 \\ & \end{array}$

Note: UPV* factors are reported for years 26-30 to accommodate a planning/construction period of up to 5 years.

See p. 14 for instructions on use; page xiii for abbreviations. 
Table Ba-4. FEMP UPV* Discount Factors adjusted for fuel price escalation, by end-use sector and fuel type.

Discount Rate $=3.0 \%(\mathrm{DOE})$

Census Region 4 (Alaska, Arizona, California, Colorado, Hawaii,

Idaho, Montana, Nevada, New Mexico, Oregon, Utah, Washington, Wyoming)

\begin{tabular}{|c|c|c|c|c|c|}
\hline & & & RESIDEN & ГIAL & \\
\hline & $\mathrm{N}$ & Elec & Dist & LPG & NtGas \\
\hline & & - - - - & & - - - - & \\
\hline & 1 & 0.97 & 0.93 & 1.00 & 0.95 \\
\hline & 2 & 1.90 & 1.79 & 1.96 & 1.84 \\
\hline & 3 & 2.79 & 2.58 & 2.88 & 2.68 \\
\hline & 4 & 3.63 & 3.29 & 3.76 & 3.47 \\
\hline & 5 & 4.43 & 3.95 & 4.60 & 4.23 \\
\hline & 6 & 5.21 & 4.55 & 5.42 & 4.95 \\
\hline & 7 & 5.97 & 5.12 & 6.21 & 5.65 \\
\hline & 8 & 6.70 & 5.68 & 6.97 & 6.32 \\
\hline & 9 & 7.41 & 6.22 & 7.72 & 6.98 \\
\hline & 10 & 8.11 & 6.75 & 8.44 & 7.64 \\
\hline & 11 & 8.79 & 7.27 & 9.14 & 8.26 \\
\hline & 12 & 9.45 & 7.79 & 9.82 & 8.85 \\
\hline & 13 & 10.09 & 8.30 & 10.47 & 9.43 \\
\hline & 14 & 10.71 & 8.80 & 11.11 & 10.00 \\
\hline$m$ & 15 & 11.32 & 9.29 & 11.74 & 10.56 \\
\hline$\infty$ & 16 & 11.90 & 9.77 & 12.35 & 11.11 \\
\hline & 17 & 12.48 & 10.24 & 12.94 & 11.65 \\
\hline & 18 & 13.04 & 10.71 & 13.52 & 12.19 \\
\hline & 19 & 13.58 & 11.17 & 14.08 & 12.70 \\
\hline & 20 & 14.11 & 11.62 & 14.63 & 13.21 \\
\hline & 21 & 14.63 & 12.06 & 15.17 & 13.71 \\
\hline & 22 & 15.13 & 12.49 & 15.69 & 14.20 \\
\hline & 23 & 15.62 & 12.91 & 16.20 & 14.68 \\
\hline & 24 & 16.10 & 13.33 & 16.69 & 15.15 \\
\hline & 25 & 16.57 & 13.73 & 17.17 & 15.61 \\
\hline & 26 & 17.02 & 14.12 & 17.64 & 16.05 \\
\hline & 27 & 17.47 & 14.49 & 18.09 & 16.49 \\
\hline & 28 & 17.90 & 14.86 & 18.53 & 16.92 \\
\hline & 29 & 18.32 & 15.22 & 18.96 & 17.35 \\
\hline & 30 & 18.73 & 15.57 & 19.38 & 17.76 \\
\hline
\end{tabular}

\begin{tabular}{rrrrr}
\multicolumn{5}{c}{ COMMERCIAL } \\
Elec & Dist & Resid & NtGas & Coal \\
$-1 .-$ &.---1 &.---- &..--- &..-- \\
0.97 & 0.92 & 0.99 & 0.94 & 0.98 \\
1.89 & 1.76 & 1.90 & 1.82 & 1.94 \\
2.76 & 2.53 & 2.69 & 2.65 & 2.88 \\
3.59 & 3.24 & 3.39 & 3.43 & 3.78 \\
4.37 & 3.90 & 4.03 & 4.17 & 4.66 \\
5.12 & 4.51 & 4.62 & 4.87 & 5.51 \\
5.85 & 5.08 & 5.16 & 5.55 & 6.34 \\
6.56 & 5.64 & 5.69 & 6.20 & 7.15 \\
7.25 & 6.18 & 6.21 & 6.84 & 7.94 \\
7.93 & 6.72 & 6.71 & 7.46 & 8.71 \\
8.58 & 7.24 & 7.22 & 8.06 & 9.47 \\
9.22 & 7.77 & 7.71 & 8.63 & 10.21 \\
9.83 & 8.28 & 8.21 & 9.18 & 10.94 \\
10.42 & 8.77 & 8.71 & 9.72 & 11.65 \\
11.01 & 9.27 & 9.20 & 10.25 & 12.35 \\
11.57 & 9.75 & 9.66 & 10.77 & 13.04 \\
12.12 & 10.21 & 10.13 & 11.28 & 13.72 \\
12.65 & 10.68 & 10.58 & 11.79 & 14.38 \\
13.17 & 11.13 & 11.03 & 12.27 & 15.02 \\
13.68 & 11.57 & 11.47 & 12.75 & 15.66 \\
14.17 & 12.01 & 11.90 & 13.22 & 16.28 \\
14.65 & 12.44 & 12.33 & 13.68 & 16.88 \\
15.12 & 12.86 & 12.74 & 14.14 & 17.47 \\
15.57 & 13.27 & 13.15 & 14.59 & 18.05 \\
16.02 & 13.66 & 13.55 & 15.03 & 18.61 \\
16.45 & 14.05 & 13.93 & 15.47 & 19.16 \\
16.87 & 14.43 & 14.30 & 15.89 & 19.70 \\
17.28 & 14.79 & 14.67 & 16.30 & 20.22 \\
17.67 & 15.15 & 15.02 & 16.71 & 20.73 \\
18.06 & 15.49 & 15.37 & 17.11 & 21.23
\end{tabular}

\begin{tabular}{|c|c|c|c|c|}
\hline ec & ist & Resid & NtGas & Coal \\
\hline & & & & \\
\hline$\odot .98$ & $\odot .91$ & 1.00 & 0.97 & 0.98 \\
\hline 1.91 & 1.74 & 1.94 & 1.87 & 1.92 \\
\hline 2.81 & 51 & 2.79 & 2.70 & 2.84 \\
\hline 3.64 & 3.23 & 3.56 & 3.46 & 3.74 \\
\hline 4.44 & 3.90 & 4.28 & 4.19 & 4.59 \\
\hline 5.21 & 4.54 & 4.9 & 4.87 & 5.42 \\
\hline 5.95 & 5.14 & 5.58 & 5.52 & 6.22 \\
\hline 6.67 & 571 & 6.1 & 6.16 & 7.00 \\
\hline 7.37 & .28 & 6.7 & 6.78 & 7.76 \\
\hline 8.06 & 6.84 & 7.3 & 7.39 & 8.51 \\
\hline 8.74 & 7.39 & 7.9 & 7.97 & 9.24 \\
\hline 38 & 7.9 & 8.5 & 8.52 & 9.95 \\
\hline 0 & .4 & 9. & 9. & 10.66 \\
\hline & 8.97 & 9.6 & 9.58 & 1.36 \\
\hline & 9.47 & & & 2.05 \\
\hline .80 & .96 & 9 & 0.62 & 2.73 \\
\hline 12.37 & .44 & 11.21 & 11.13 & 13.39 \\
\hline 2.92 & & 11.72 & 11.63 & 14.04 \\
\hline .45 & .37 & 2.2 & 12.12 & 14.68 \\
\hline .98 & .82 & 12.71 & 12.60 & 15.30 \\
\hline 4.48 & 12.27 & 13.19 & 13.07 & 15.91 \\
\hline & & & & 16.51 \\
\hline 5.47 & .13 & 14. & 14.00 & 17.10 \\
\hline 15.94 & 13.55 & 14.57 & 14.45 & 17.67 \\
\hline$\because 1$ & & & 14.89 & 18.23 \\
\hline & & & & \\
\hline 17.29 & 14.73 & 15.83 & 15.76 & 19.30 \\
\hline & & 16. & 16.18 & 19.82 \\
\hline & & 16.62 & 16.59 & 20.32 \\
\hline & & & & \\
\hline
\end{tabular}

$\begin{array}{rr}\text { TRANSPORT } & \\ \text { Gasln } & \mathrm{N} \\ ---- & - \\ 0.93 & 1 \\ 1.80 & 2 \\ 2.61 & 3 \\ 3.37 & 4 \\ 4.09 & 5 \\ 4.78 & 6 \\ 5.43 & 7 \\ 6.06 & 8 \\ 6.67 & 9 \\ 7.27 & 10 \\ 7.85 & 11 \\ 8.42 & 12 \\ 8.98 & 13 \\ 9.53 & 14 \\ 10.06 & 15 \\ 10.58 & 16 \\ 11.09 & 17 \\ 11.59 & 18 \\ 12.08 & 19 \\ 12.55 & 20 \\ 13.01 & 21 \\ 13.46 & 22 \\ 13.90 & 23 \\ 14.33 & 24 \\ 14.75 & 25 \\ 15.16 & 26 \\ 15.55 & 27 \\ 15.94 & 28 \\ 16.31 & 29 \\ 16.67 & 30\end{array}$

Note: UPV* factors are reported for years 26-30 to accommodate a planning/construction period of up to 5 years.

See p. 14 for instructions on use; page xiii for abbreviations. 
Table Ba-5. FEMP UPV* Discount Factors adjusted for fuel price escalation, by end-use sector and fuel type.

Discount Rate $=3.0 \%(\mathrm{DOE})$

United States Average

\begin{tabular}{rrrrr}
\multicolumn{5}{c}{ RESIDENTIAL } \\
$\mathrm{N}$ & \multicolumn{1}{c}{ Elec } & \multicolumn{1}{l}{ Dist } & \multicolumn{1}{l}{ LPG } & NtGas \\
- &.---1 &.---1 &..-- &..--- \\
1 & 0.97 & 0.96 & 1.01 & 0.94 \\
2 & 1.90 & 1.83 & 1.97 & 1.83 \\
3 & 2.79 & 2.63 & 2.88 & 2.66 \\
4 & 3.65 & 3.36 & 3.76 & 3.44 \\
5 & 4.48 & 4.04 & 4.61 & 4.19 \\
6 & 5.28 & 4.65 & 5.42 & 4.90 \\
7 & 6.05 & 5.24 & 6.21 & 5.59 \\
8 & 6.79 & 5.82 & 6.97 & 6.26 \\
9 & 7.52 & 6.38 & 7.72 & 6.91 \\
10 & 8.23 & 6.92 & 8.44 & 7.56 \\
11 & 8.92 & 7.46 & 9.15 & 8.18 \\
12 & 9.59 & 7.98 & 9.84 & 8.79 \\
13 & 10.24 & 8.50 & 10.50 & 9.38 \\
14 & 10.88 & 9.00 & 11.15 & 9.95 \\
15 & 11.49 & 9.50 & 11.78 & 10.52 \\
16 & 12.09 & 9.98 & 12.40 & 11.07 \\
17 & 12.68 & 10.45 & 13.00 & 11.62 \\
18 & 13.24 & 10.91 & 13.58 & 12.15 \\
19 & 13.79 & 11.36 & 14.15 & 12.67 \\
20 & 14.33 & 11.80 & 14.70 & 13.17 \\
21 & 14.85 & 12.23 & 15.24 & 13.67 \\
22 & 15.35 & 12.65 & 15.77 & 14.16 \\
23 & 15.85 & 13.07 & 16.28 & 14.63 \\
24 & 16.32 & 13.47 & 16.78 & 15.10 \\
25 & 16.79 & 13.86 & 17.26 & 15.56 \\
26 & 17.25 & 14.24 & 17.73 & 16.00 \\
27 & 17.69 & 14.61 & 18.19 & 16.44 \\
28 & 18.12 & 14.97 & 18.63 & 16.86 \\
29 & 18.53 & 15.31 & 19.06 & 17.28 \\
30 & 18.94 & 15.65 & 19.47 & 17.69
\end{tabular}

\begin{tabular}{rrrrr}
\multicolumn{5}{c}{ COMMERCIAL } \\
Elec & \multicolumn{1}{c}{ Dist } & Resid & NtGas & Coal \\
.---- &.--- &.--- &..-- &.--- \\
0.97 & 0.94 & 0.91 & 0.94 & 0.98 \\
1.89 & 1.79 & 1.77 & 1.81 & 1.93 \\
2.78 & 2.57 & 2.60 & 2.63 & 2.85 \\
3.62 & 3.29 & 3.36 & 3.39 & 3.75 \\
4.42 & 3.94 & 4.08 & 4.11 & 4.62 \\
5.19 & 4.55 & 4.70 & 4.79 & 5.45 \\
5.94 & 5.12 & 5.30 & 5.45 & 6.25 \\
6.66 & 5.68 & 5.91 & 6.09 & 7.02 \\
7.37 & 6.23 & 6.50 & 6.71 & 7.77 \\
8.06 & 6.77 & 7.08 & 7.32 & 8.49 \\
8.74 & 7.29 & 7.66 & 7.92 & 9.19 \\
9.40 & 7.81 & 8.23 & 8.49 & 9.86 \\
10.04 & 8.32 & 8.81 & 9.05 & 10.52 \\
10.66 & 8.82 & 9.38 & 9.59 & 11.16 \\
11.27 & 9.31 & 9.94 & 10.12 & 11.79 \\
11.86 & 9.79 & 10.48 & 10.64 & 12.39 \\
12.43 & 10.25 & 11.01 & 11.16 & 12.99 \\
12.99 & 10.71 & 11.52 & 11.66 & 13.57 \\
13.53 & 11.16 & 12.01 & 12.14 & 14.13 \\
14.06 & 11.60 & 12.50 & 12.62 & 14.69 \\
14.57 & 12.03 & 12.98 & 13.08 & 15.23 \\
15.07 & 12.45 & 13.43 & 13.54 & 15.76 \\
15.55 & 12.87 & 13.87 & 13.99 & 16.27 \\
16.02 & 13.27 & 14.30 & 14.42 & 16.77 \\
16.48 & 13.67 & 14.72 & 14.85 & 17.27 \\
16.92 & 14.05 & 15.13 & 15.27 & 17.74 \\
17.36 & 14.42 & 15.52 & 15.68 & 18.21 \\
17.78 & 14.79 & 15.91 & 16.08 & 18.66 \\
18.19 & 15.14 & 16.28 & 16.48 & 19.11 \\
18.58 & 15.48 & 16.65 & 16.86 & 19.54
\end{tabular}

\begin{tabular}{rrrrr}
\multicolumn{5}{c}{ INDUSTRIAL } \\
Elec & Dist & Resid & NtGas & \multicolumn{1}{c}{ Coal } \\
.---- &.---- &.---- &.--- &.--- \\
0.98 & 0.92 & 0.99 & 0.96 & 0.98 \\
1.92 & 1.76 & 1.92 & 1.84 & 1.93 \\
2.81 & 2.54 & 2.78 & 2.65 & 2.85 \\
3.64 & 3.26 & 3.58 & 3.38 & 3.75 \\
4.43 & 3.94 & 4.32 & 4.07 & 4.61 \\
5.17 & 4.58 & 5.01 & 4.72 & 5.44 \\
5.89 & 5.18 & 5.66 & 5.35 & 6.24 \\
6.59 & 5.77 & 6.30 & 5.96 & 7.02 \\
7.27 & 6.35 & 6.93 & 6.55 & 7.78 \\
7.94 & 6.91 & 7.54 & 7.14 & 8.51 \\
8.60 & 7.46 & 8.14 & 7.72 & 9.21 \\
9.24 & 8.01 & 8.74 & 8.27 & 9.90 \\
9.86 & 8.54 & 9.33 & 8.82 & 10.56 \\
10.47 & 9.06 & 9.91 & 9.35 & 11.21 \\
11.06 & 9.58 & 10.49 & 9.86 & 11.85 \\
11.63 & 10.07 & 11.04 & 10.38 & 12.47 \\
12.20 & 10.56 & 11.58 & 10.89 & 13.07 \\
12.74 & 11.04 & 12.11 & 11.38 & 13.66 \\
13.27 & 11.51 & 12.63 & 11.86 & 14.23 \\
13.79 & 11.97 & 13.13 & 12.34 & 14.79 \\
14.28 & 12.42 & 13.63 & 12.80 & 15.33 \\
14.77 & 12.86 & 14.11 & 13.26 & 15.86 \\
15.25 & 13.30 & 14.57 & 13.71 & 16.38 \\
15.71 & 13.72 & 15.03 & 14.15 & 16.89 \\
16.16 & 14.14 & 15.48 & 14.59 & 17.38 \\
16.59 & 14.54 & 15.91 & 15.01 & 17.86 \\
17.02 & 14.93 & 16.33 & 15.43 & 18.33 \\
17.43 & 15.31 & 16.73 & 15.84 & 18.79 \\
17.83 & 15.67 & 17.13 & 16.24 & 19.23 \\
18.22 & 16.03 & 17.52 & 16.64 & 19.67 \\
& & & &
\end{tabular}

$\begin{array}{rr}\text { TRANSPORT } & \\ \text { Gasln } & \mathrm{N} \\ --\cdot- & - \\ 0.93 & 1 \\ 1.77 & 2 \\ 2.56 & 3 \\ 3.30 & 4 \\ 3.99 & 5 \\ 4.66 & 6 \\ 5.29 & 7 \\ 5.89 & 8 \\ 6.48 & 9 \\ 7.05 & 10 \\ 7.61 & 11 \\ 8.16 & 12 \\ 8.70 & 13 \\ 9.23 & 14 \\ 9.75 & 15 \\ 10.25 & 16 \\ 10.74 & 17 \\ 11.22 & 18 \\ 11.69 & 19 \\ 12.15 & 20 \\ 12.59 & 21 \\ 13.03 & 22 \\ 13.45 & 23 \\ 13.87 & 24 \\ 14.27 & 25 \\ 14.67 & 26 \\ 15.05 & 27 \\ 15.42 & 28 \\ 15.78 & 29 \\ 16.13 & 30 \\ & \end{array}$

Note: UPV* factors are reported for years 26-30 to accommodate a planning/construction period of up to 5 years.

See p. 14 for instructions on use; page xiii for abbreviations. 


\section{B.2. FEMP Modified Uniform Present Value Factors}

The OMB Modified Uniform Present Value (OMB UPV*) factors presented in the "Bb" tables, based on the current OMB discount rates (2.7 \% short term and $3.0 \%$ long term), are for calculating the present value of energy costs accruing over 1 to 30 years when conducting a lifecycle cost analysis of a federal project not explicitly related to energy or water conservation or renewable resources. Factors are reported in the "Bb" tables for 30 years. These factors apply only to annual energy usage that is assumed to be the same each year over the service period. The NIST BLCC computer program can compute the present value of energy usage and savings that are not the same in each year.

\section{Examples of How to Use the OMB UPV* Factors:}

OMB UPV* (OMB discount rate): To compute the present value over 30 years of electricity costs associated with the occupancy of a federal office building in Ohio (where energy conservation is not a specific consideration in the LCC analysis), go to Table Bb-2, find the OMB UPV* factor for commercial electricity for 30 years (19.96), and multiply this factor by the annual electricity cost in base-date dollars.

Note: Because the discount rate used to calculate the Bb tables (OMB discount rate) is usually different for years 1 to 10 than for years 11 to 30, these factors cannot be used with a planning/ design/construction period as shown above for the Ba tables (DOE discount rate). Use the BLCC or DISCOUNT computer program for this purpose. For further explanation of the use of UPV* factors, see NIST Handbook 135. 
Table Bb-1. OMB UPV* Discount Factors adjusted for fuel price escalation, by end-use sector and fuel type.

Discount Rate $=2.7 \%$ (years 1 to 10$)$ and $3.0 \%$ (years 11 to 30), (OMB Circular A-94 ${ }^{\mathrm{a}}$ )

Census Region 1 (Connecticut, Maine, Massachusetts, New Hampshire,

New Jersey, New York, Pennsylvania, Rhode Island, Vermont)

\begin{tabular}{|c|c|c|c|c|}
\hline \multicolumn{5}{|c|}{ COMMERCIAL } \\
\hline Elec & Dist & Resid & NtGas & Coal \\
\hline & 0 & 0 & $0 \Omega$ & 097 \\
\hline & 1.81 & 1.74 & 1.81 & 1.91 \\
\hline 2.67 & 2.59 & 2.57 & 2.63 & 2.81 \\
\hline 3.45 & 3.32 & 3.33 & 3.39 & 3.68 \\
\hline 4.21 & 3.99 & 4.05 & 4.12 & 4.51 \\
\hline 4.95 & 4.62 & 4.67 & 4.81 & 5.29 \\
\hline 5.66 & 5.21 & 5.27 & 5.48 & 6.03 \\
\hline 6.36 & 5.79 & 5.89 & 6.12 & 6.75 \\
\hline 7.05 & 6.36 & 6.49 & 6.75 & 7.43 \\
\hline 7.72 & 6.92 & 7.08 & 7.38 & 8.09 \\
\hline 8.24 & 7.35 & 7.54 & 7.86 & 8.59 \\
\hline 8.86 & 7.88 & 8.12 & 8.43 & 9.18 \\
\hline 9.47 & 8.40 & 8.69 & 8.98 & 9.76 \\
\hline 10.05 & 8.90 & 9.25 & 9.52 & 10.33 \\
\hline 10.62 & 9.40 & 9.81 & 10.05 & 10.88 \\
\hline 11.18 & 9.88 & 10.35 & 10.56 & 11.43 \\
\hline 11.72 & 10.35 & 10.88 & 11.07 & 11.96 \\
\hline 12.24 & 10.82 & 11.38 & 11.56 & 12.48 \\
\hline 12.75 & 11.27 & 11.87 & 12.04 & 12.99 \\
\hline 13.24 & 11.71 & 12.35 & 12.50 & 13.48 \\
\hline 13.73 & 12.15 & 12.83 & 12.96 & 13.97 \\
\hline 14.20 & 12.58 & 13.27 & 13.41 & 14.45 \\
\hline 14.65 & 13.00 & 13.71 & 13.85 & 14.92 \\
\hline 15.09 & 13.41 & 14.13 & 14.28 & 15.38 \\
\hline 15.52 & 13.81 & 14.54 & 14.69 & 15.83 \\
\hline 15.93 & 14.20 & 14.94 & 15.10 & 16.26 \\
\hline 16.33 & 14.57 & 15.33 & 15.50 & 16.69 \\
\hline 16.72 & 14.94 & 15.71 & 15.90 & 17.10 \\
\hline 17.10 & 15.29 & 16.08 & 16.28 & 17.50 \\
\hline & 15.64 & 16.44 & 16.66 & 17.90 \\
\hline
\end{tabular}

\begin{tabular}{|c|c|c|c|c|}
\hline \multicolumn{5}{|c|}{ INDUSTRIAL } \\
\hline lec & Dist & Resid & NtGas & Coal \\
\hline 9 & 89 & 0.93 & 5 & 98 \\
\hline $\begin{array}{l}0.9 \\
1.8\end{array}$ & 1.7 & & & \\
\hline 2.68 & 2.57 & 2.68 & 2.62 & 2.88 \\
\hline 3.41 & 3.31 & 3.48 & 3.36 & 3.81 \\
\hline 4.10 & 4.00 & 4.24 & 4.05 & 4.68 \\
\hline 4.76 & 4.65 & & & \\
\hline 5.38 & 5.27 & 5.5 & 5.34 & 6.33 \\
\hline 5.99 & 5.87 & 6.20 & 5.95 & 7.11 \\
\hline 6.59 & 6.47 & & & 7.85 \\
\hline 7.18 & 7.05 & 7.46 & 7.14 & 8.58 \\
\hline 7.64 & 7.50 & 7.95 & 7.60 & 9.12 \\
\hline 8.1 & 8.05 & & & 9.76 \\
\hline 8.71 & .59 & 9.16 & 8.67 & 10.39 \\
\hline 9.23 & 9.12 & 5 & 9. & 11.01 \\
\hline .73 & 9.64 & & 99 & 11.61 \\
\hline .22 & 10.14 & & 10. & 12. \\
\hline .70 & .63 & 5 & 57 & 12.76 \\
\hline .17 & L. 11 & & & 13.32 \\
\hline & .59 & & 1 & 13. \\
\hline .06 & 12.05 & 13.00 & 12.06 & 14.39 \\
\hline 2.49 & 12.51 & & 12.51 & 14.91 \\
\hline 01 & 12.96 & & & \\
\hline .3 & 13.40 & 14. & 13. & 15.92 \\
\hline 13.70 & 13.83 & 14.86 & 13.79 & 16.40 \\
\hline 118 & 14.25 & & & \\
\hline & 4.65 & & 14. & 17.34 \\
\hline .82 & 15.05 & 16. & 15.01 & 17.78 \\
\hline 17 & 15.43 & 16.5 & 15.40 & 18.22 \\
\hline & 5.80 & & & \\
\hline 58 & 0.17 & 17.28 & 16.15 & 19.06 \\
\hline
\end{tabular}

Gasln

0.92

1.76

2.55

3.28

3.98

4.65

5.28
5.89

6.48

7.07

7.52

8.07

8.60

9.12
9.63

9.63
10.13

10.62

11.09

11.55

12.00

12.44

12.87

13.29

13.70

14.10

14.49

14.86

15.23

$\begin{array}{llll}18.40 & 15.02 & 18.32 & 16.89\end{array}$

$\begin{array}{lllll}17.47 & 15.64 & 16.44 & 16.66 & 17.90\end{array}$

$\begin{array}{lllll}15.85 & 16.17 & 17.28 & 16.15 & 19.06\end{array}$

15.93

${ }^{\mathrm{a}}$ OMB discount rate as of February 2007.

See p. 20 for instructions on use; page xiii for abbreviations. 
Table Bb-2. OMB UPV* Discount Factors adjusted for fuel price escalation, by end-use sector and fuel type.

Discount Rate $=2.7 \%$ (years 1 to 10 ) and $3.0 \%$ (years 11 to 30), (OMB Circular A-94 $4^{\mathrm{a}}$ )

Census Region 2 (Illinois, Indiana, Iowa, Kansas, Michigan, Minnesota,

Missouri, Nebraska, North Dakota, Ohio, South Dakota, Wisconsin)

\begin{tabular}{rrrr}
\multicolumn{4}{c}{ RESIDENTIAL } \\
Elec & \multicolumn{1}{l}{ Dist } & \multicolumn{1}{l}{ LPG } & NtGas \\
.---- &.---1 &..-- &..-- \\
0.99 & 0.96 & 1.02 & 0.94 \\
1.95 & 1.83 & 2.00 & 1.83 \\
2.89 & 2.63 & 2.93 & 2.66 \\
3.81 & 3.35 & 3.83 & 3.44 \\
4.70 & 4.00 & 4.69 & 4.19 \\
5.56 & 4.60 & 5.53 & 4.91 \\
6.39 & 5.16 & 6.34 & 5.60 \\
7.20 & 5.71 & 7.13 & 6.27 \\
7.99 & 6.25 & 7.90 & 6.93 \\
8.76 & 6.78 & 8.65 & 7.59 \\
9.36 & 7.18 & 9.23 & 8.10 \\
10.07 & 7.68 & 9.92 & 8.70 \\
10.76 & 8.17 & 10.60 & 9.28 \\
11.44 & 8.64 & 11.25 & 9.86 \\
12.09 & 9.11 & 11.89 & 10.42 \\
12.73 & 9.57 & 12.51 & 10.97 \\
13.35 & 10.01 & 13.11 & 11.51 \\
13.95 & 10.45 & 13.70 & 12.04 \\
14.53 & 10.87 & 14.28 & 12.56 \\
15.09 & 11.28 & 14.83 & 13.06 \\
15.64 & 11.69 & 15.38 & 13.56 \\
16.17 & 12.08 & 15.91 & 14.05 \\
16.68 & 12.47 & 16.43 & 14.52 \\
17.19 & 12.85 & 16.93 & 14.99 \\
17.67 & 13.22 & 17.42 & 15.44 \\
18.15 & 13.57 & 17.89 & 15.89 \\
18.61 & 13.92 & 18.35 & 16.32 \\
19.06 & 14.26 & 18.80 & 16.75 \\
19.49 & 14.59 & 19.24 & 17.16 \\
19.91 & 14.90 & 19.66 & 17.57
\end{tabular}

\begin{tabular}{|c|c|c|c|c|}
\hline \multicolumn{5}{|c|}{ COMMERCIAL } \\
\hline Elec & Dist & Resid & NtGas & Coal \\
\hline & & & & \\
\hline 0.99 & 0.93 & 1.06 & 0.94 & 0.99 \\
\hline 1.96 & 1.78 & 2.06 & 1.82 & 1.95 \\
\hline 2.90 & 2.56 & 2.98 & 2.64 & 2.92 \\
\hline 3.82 & 3.27 & 3.85 & 3.41 & 3.87 \\
\hline 4.69 & 3.92 & 4.65 & 4.14 & 4.80 \\
\hline 5.53 & 4.52 & 5.41 & 4.83 & 5.70 \\
\hline 6.34 & 5.09 & 6.14 & 5.50 & 6.58 \\
\hline 7.13 & 5.64 & 6.85 & 6.15 & 7.42 \\
\hline 7.90 & 6.19 & 7.54 & 6.79 & 8.24 \\
\hline 8.66 & 6.72 & 8.22 & 7.42 & 9.03 \\
\hline 9.26 & 7.13 & 8.74 & 7.91 & 9.63 \\
\hline 9.97 & 7.64 & 9.38 & 8.48 & 10.35 \\
\hline 10.66 & 8.13 & 10.00 & 9.05 & 11.05 \\
\hline 11.33 & 8.61 & 10.62 & 9.59 & 11.72 \\
\hline .99 & 9.09 & 11.22 & 10.13 & 12.38 \\
\hline 12.63 & 9.55 & 11.81 & 10.65 & 13.01 \\
\hline 13.26 & 10.00 & 12.38 & 11.17 & 13.64 \\
\hline .88 & 10.44 & 12.94 & 11.67 & 14.24 \\
\hline 14.47 & 10.87 & 13.48 & 12.16 & 14.84 \\
\hline 15.04 & 11.30 & 14.01 & 12.64 & 15.42 \\
\hline 50 & 11.72 & 14.53 & 13.11 & 15.98 \\
\hline 0.14 & 12.13 & 15.04 & 13.57 & 16.53 \\
\hline 16.66 & 12.53 & 15.54 & 14.02 & 17.07 \\
\hline 17.17 & 12.92 & 16.03 & 14.46 & 17.60 \\
\hline 17.67 & 13.30 & 16.50 & 14.89 & 18.11 \\
\hline 18.15 & 13.67 & 16.96 & 15.31 & 18.60 \\
\hline 18.62 & 14.03 & 17.40 & 15.72 & 19.09 \\
\hline & 14.38 & 17.84 & 16.13 & \\
\hline 19.52 & 14.71 & 18.26 & 16.52 & 20.02 \\
\hline 19.96 & 15.04 & 18.67 & 16.91 & 20.48 \\
\hline
\end{tabular}

\begin{tabular}{|c|c|c|c|c|}
\hline \multicolumn{5}{|c|}{ INDUSTRIAL } \\
\hline Elec & Dist & Resid & NtGas & Coal \\
\hline 1.01 & 0.92 & 1.06 & 0.97 & 0.98 \\
\hline 1.99 & 1.76 & 2.05 & 1.87 & 1.94 \\
\hline 2.93 & 2.55 & 2.97 & 2.71 & 2.90 \\
\hline 3.81 & 3.28 & 3.83 & 3.48 & 3.84 \\
\hline 4.63 & 3.97 & 4.63 & 4.22 & 4.75 \\
\hline 5.42 & 4.62 & 5.39 & 4.90 & 5.63 \\
\hline 6.18 & 5.24 & 6.11 & 5.57 & 6.49 \\
\hline 6.91 & 5.84 & 6.81 & 6.22 & 7.32 \\
\hline 7.63 & 6.44 & 7.50 & 6.85 & 8.13 \\
\hline 8.35 & 7.02 & 8.18 & 7.49 & 8.91 \\
\hline 8.90 & 7.47 & 8.70 & 7.98 & 9.51 \\
\hline 9.57 & 8.02 & 9.33 & 8.56 & 10.23 \\
\hline 10.22 & 8.55 & 9.95 & 9.12 & 10.93 \\
\hline 10.86 & 9.08 & 10.56 & 9.67 & 11.61 \\
\hline 11.47 & 9.59 & 11.17 & 10.21 & 12.27 \\
\hline 12.08 & 10.09 & 11.75 & 10.74 & 12.92 \\
\hline 12.67 & 10.57 & 12.32 & 11.27 & 13.55 \\
\hline 13.25 & 11.05 & 12.87 & 11.78 & 14.16 \\
\hline 13.80 & 11.52 & 13.41 & 12.27 & 14.77 \\
\hline 14.34 & 11.98 & 13.94 & 12.76 & 15.35 \\
\hline 14.87 & 12.43 & 14.46 & 13.24 & 15.93 \\
\hline 15.38 & 12.87 & 14.97 & 13.71 & 16.49 \\
\hline 15.87 & 13.31 & 15.47 & 14.17 & 17.03 \\
\hline 16.35 & 13.73 & 15.95 & 14.63 & 17.57 \\
\hline 16.82 & 14.14 & 16.42 & 15.07 & 18.09 \\
\hline 17.28 & 14.54 & 16.87 & 15.51 & 18.59 \\
\hline 17.73 & 14.93 & 17.32 & 15.93 & 19.09 \\
\hline 18.16 & 15.31 & 17.75 & 16.35 & 19.57 \\
\hline 18.58 & 15.67 & 18.17 & 16.77 & 20.04 \\
\hline 18.99 & 16.03 & 18.58 & 17.17 & 20.50 \\
\hline
\end{tabular}

Gasln

0.93

1.79

2.58

3.32

4.02

4.69

5.33
5.94

6.54

7.12

7.57

8.12

8.66

9.18

9.69

10.19

10.68

11.16

11.62

12.07

12.51

12.94

13.36

13.78

14.18

14.57

14.94

15.31

15.67

16.02

${ }^{\mathrm{a}}$ OMB discount rate as of February 2007.

See p. 20 for instructions on use; page xiii for abbreviations. 


\section{Table Bb-3. OMB UPV* Discount Factors adjusted for fuel price escalation, by end-use sector and fuel type.}

Discount Rate $=2.7 \%$ (years 1 to 10 ) and $3.0 \%$ (years 11 to 30), (OMB Circular A-94 $4^{\mathrm{a}}$ )

Census Region 3 (Alabama, Arkansas, Delaware, District of Columbia, Florida, Georgia, Kentucky, Louisiana, Maryland, Mississippi, North Carolina, Oklahoma, South Carolina, Tennessee, Texas, Virginia, West Virginia)

\begin{tabular}{|c|c|c|c|c|}
\hline & & RESIDEN & IIAL & \\
\hline $\mathrm{N}$ & Elec & Dist & LPG & NtGas \\
\hline - & - - - - & - - - - & - - - - & - - - - - \\
\hline 1 & 0.97 & 0.96 & $1 . \odot \odot$ & 0.95 \\
\hline 2 & 1.90 & 1.85 & 1.96 & 1.84 \\
\hline 3 & 2.80 & 2.65 & 2.88 & 2.68 \\
\hline 4 & 3.66 & 3.40 & 3.76 & 3.47 \\
\hline 5 & 4.48 & 4.08 & 4.61 & 4.23 \\
\hline 6 & 5.28 & 4.72 & 5.43 & 4.96 \\
\hline 7 & 6.05 & 5.32 & 6.23 & 5.67 \\
\hline 8 & 6.80 & 5.91 & 7.01 & 6.35 \\
\hline 9 & 7.53 & 6.48 & 7.76 & 7.03 \\
\hline 10 & 8.24 & 7.05 & 8.51 & 7.69 \\
\hline 11 & 8.80 & 7.49 & 9.08 & 8.21 \\
\hline 12 & 9.46 & 8.01 & 9.76 & 8.83 \\
\hline 13 & 10.10 & 8.53 & 10.43 & 9.44 \\
\hline 14 & 10.72 & 9.04 & 11.07 & 10.02 \\
\hline 15 & 11.33 & 9.54 & 11.70 & 10.60 \\
\hline 16 & 11.91 & 10.02 & 12.31 & 11.16 \\
\hline 17 & 12.49 & 10.50 & 12.91 & 11.72 \\
\hline 18 & 13.04 & 10.96 & 13.49 & 12.26 \\
\hline 19 & 13.58 & 11.41 & 14.05 & 12.78 \\
\hline 20 & 14.11 & 11.85 & 14.60 & 13.30 \\
\hline 21 & 14.62 & 12.29 & 15.13 & 13.80 \\
\hline 22 & 15.11 & 12.71 & 15.65 & 14.30 \\
\hline 23 & 15.60 & 13.13 & 16.16 & 14.78 \\
\hline 24 & 16.06 & 13.53 & 16.65 & 15.25 \\
\hline 25 & 16.52 & 13.92 & 17.13 & 15.71 \\
\hline 26 & 16.96 & 14.30 & 17.59 & 16.16 \\
\hline 27 & 17.39 & 14.67 & 18.04 & 16.60 \\
\hline 28 & 17.81 & 15.03 & 18.48 & 17.03 \\
\hline 29 & 18.22 & 15.38 & 18.91 & 17.45 \\
\hline 30 & 18.62 & 15.72 & 19.32 & 17.86 \\
\hline
\end{tabular}

\begin{tabular}{|c|c|c|c|c|}
\hline \multicolumn{5}{|c|}{ COMMERCIAL } \\
\hline Elec & Dist & Resid & NtGas & Coal \\
\hline 0.97 & 0.95 & 0.99 & 0.95 & 0.98 \\
\hline 1.91 & 1.81 & 1.91 & 1.83 & 1.93 \\
\hline 2.82 & 2.59 & 2.75 & 2.65 & 2.84 \\
\hline 3.68 & 3.31 & 3.52 & 3.42 & 3.71 \\
\hline 4.51 & 3.98 & 4.24 & 4.14 & 4.54 \\
\hline 5.31 & 4.59 & 4.92 & 4.83 & 5.33 \\
\hline 6.09 & 5.17 & 5.55 & 5.50 & 6.11 \\
\hline 6.84 & 5.73 & 6.17 & 6.15 & 6.86 \\
\hline 7.58 & 6.29 & 6.78 & 6.79 & 7.60 \\
\hline 8.30 & 6.84 & 7.38 & 7.42 & 8.31 \\
\hline 8.87 & 7.26 & 7.84 & 7.91 & 8.85 \\
\hline 9.54 & 7.78 & 8.40 & 8.49 & 9.48 \\
\hline 10.20 & 8.29 & 8.95 & 9.05 & 10.09 \\
\hline 10.83 & 8.78 & 9.50 & 9.60 & 10.68 \\
\hline 11.45 & 9.27 & 10.05 & 10.14 & 11.26 \\
\hline 12.05 & 9.74 & 10.57 & 10.67 & 11.82 \\
\hline 12.64 & 10.21 & 11.08 & 11.18 & 12.37 \\
\hline 13.21 & 10.66 & 11.59 & 11.69 & 12.90 \\
\hline 13.76 & 11.11 & 12.08 & 12.18 & 13.42 \\
\hline 14.30 & 11.54 & 12.56 & 12.66 & 13.92 \\
\hline 14.82 & 11.98 & 13.03 & 13.13 & 14.40 \\
\hline 15.33 & $12.4 \odot$ & 13.50 & 13.59 & 14.88 \\
\hline 15.82 & 12.81 & 13.95 & 14.04 & 15.34 \\
\hline 16.30 & 13.22 & 14.40 & 14.47 & 15.80 \\
\hline 16.77 & 13.61 & 14.83 & 14.90 & 16.24 \\
\hline 17.22 & 13.99 & 15.25 & 15.32 & 16.67 \\
\hline 17.66 & 14.36 & 15.65 & 15.73 & 17.09 \\
\hline 18.09 & 14.72 & 16.05 & 16.13 & 17.50 \\
\hline 18.50 & 15.07 & 16.44 & 16.53 & 17.89 \\
\hline 18.91 & 15.41 & 16.81 & 16.91 & 18.28 \\
\hline
\end{tabular}

\begin{tabular}{rrrrr}
\multicolumn{5}{c}{ INDUSTRIAL } \\
Elec & \multicolumn{1}{c}{ Dist } & Resid & NtGas & Coal \\
..--- &.--- &.--- &.--- &.--- \\
0.98 & 0.93 & 1.02 & 0.96 & 0.98 \\
1.92 & 1.78 & 1.96 & 1.83 & 1.92 \\
2.83 & 2.56 & 2.83 & 2.62 & 2.83 \\
3.68 & 3.30 & 3.63 & 3.34 & 3.72 \\
4.48 & 3.99 & 4.38 & 4.01 & 4.56 \\
5.26 & 4.63 & 5.09 & 4.65 & 5.38 \\
6.01 & 5.25 & 5.76 & 5.26 & 6.18 \\
6.74 & 5.85 & 6.41 & 5.86 & 6.95 \\
7.45 & 6.44 & 7.05 & 6.44 & 7.70 \\
8.15 & 7.02 & 7.68 & 7.03 & 8.43 \\
8.70 & 7.47 & 8.16 & 7.49 & 8.98 \\
9.36 & 8.02 & 8.75 & 8.04 & 9.64 \\
9.99 & 8.55 & 9.33 & 8.57 & 10.28 \\
10.61 & 9.08 & 9.90 & 9.09 & 10.90 \\
11.21 & 9.60 & 10.47 & 9.60 & 11.50 \\
11.80 & 10.10 & 11.01 & 10.10 & 12.09 \\
12.37 & 10.58 & 11.55 & 10.61 & 12.66 \\
12.93 & 11.07 & 12.07 & 11.09 & 13.21 \\
13.46 & 11.54 & 12.58 & 11.57 & 13.76 \\
13.99 & 12.00 & 13.09 & 12.03 & 14.28 \\
14.50 & 12.45 & 13.57 & 12.49 & 14.79 \\
14.99 & 12.90 & 14.06 & 12.94 & 15.29 \\
15.47 & 13.34 & 14.53 & 13.39 & 15.78 \\
15.94 & 13.77 & 14.98 & 13.82 & 16.26 \\
16.39 & 14.18 & 15.43 & 14.24 & 16.72 \\
16.83 & 14.58 & 15.86 & 14.66 & 17.17 \\
17.26 & 14.98 & 16.28 & 15.08 & 17.61 \\
17.68 & 15.36 & 16.69 & 15.48 & 18.04 \\
18.09 & 15.73 & 17.09 & 15.88 & 18.46 \\
18.48 & 16.09 & 17.47 & 16.27 & 18.86 \\
& & & &
\end{tabular}

$\begin{array}{rr}\text { TRANSPORT } & \\ \text { Gasln } & \mathrm{N} \\ --.- & - \\ 0.93 & 1 \\ 1.77 & 2 \\ 2.56 & 3 \\ 3.30 & 4 \\ 4.00 & 5 \\ 4.66 & 6 \\ 5.30 & 7 \\ 5.91 & 8 \\ 6.50 & 9 \\ 7.08 & 10 \\ 7.53 & 11 \\ 8.08 & 12 \\ 8.61 & 13 \\ 9.14 & 14 \\ 9.65 & 15 \\ 10.15 & 16 \\ 10.63 & 17 \\ 11.11 & 18 \\ 11.57 & 19 \\ 12.02 & 20 \\ 12.46 & 21 \\ 12.90 & 22 \\ 13.32 & 23 \\ 13.73 & 24 \\ 14.13 & 25 \\ 14.52 & 26 \\ 14.90 & 27 \\ 15.27 & 28 \\ 15.62 & 29 \\ 15.97 & 30 \\ & \end{array}$

${ }^{\mathrm{a}} \mathrm{OMB}$ discount rate as of February 2007

See p. 20 for instructions on use; page xiii for abbreviations. 
Table Bb-4. OMB UPV* Discount Factors adjusted for fuel price escalation, by end-use sector and fuel type.

Discount Rate $=2.7 \%$ (years 1 to 10$)$ and $3.0 \%$ (years 11 to 30), (OMB Circular A-94 ${ }^{\mathrm{a}}$ )

Census Region 4 (Alaska, Arizona, California, Colorado, Hawaii,

Idaho, Montana, Nevada, New Mexico, Oregon, Utah, Washington, Wyoming)

\begin{tabular}{|c|c|c|c|c|}
\hline \multicolumn{5}{|c|}{ COMMERCIAL } \\
\hline Elec & Dist & Resid & NtGas & Coal \\
\hline & & & & \\
\hline$\odot .97$ & 0.92 & 0.99 & $\odot .94$ & 0.98 \\
\hline 1.89 & 1.77 & 1.91 & 1.83 & 1.95 \\
\hline 2.78 & 2.55 & 2.71 & 2.67 & 2.89 \\
\hline 3.61 & 3.27 & 3.41 & 3.45 & 3.81 \\
\hline 4.40 & 3.93 & 4.07 & 4.21 & 4.70 \\
\hline 5.17 & 4.55 & 4.66 & 4.92 & 5.57 \\
\hline 5.92 & 5.14 & 5.22 & 5.61 & 6.42 \\
\hline 6.64 & 5.70 & 5.76 & 6.28 & 7.24 \\
\hline 7.35 & 6.26 & 6.29 & 6.93 & 8.06 \\
\hline 8.05 & 6.82 & 6.81 & 7.58 & 8.85 \\
\hline 8.58 & 7.24 & 7.22 & 8.06 & 9.47 \\
\hline 9.22 & 7.77 & 7.71 & 8.63 & 10.21 \\
\hline 9.83 & 8.28 & 8.21 & 9.18 & 10.94 \\
\hline 10.42 & 8.77 & 8.71 & 9.72 & 11.65 \\
\hline 11.01 & 9.27 & 9.20 & 10.25 & 12.35 \\
\hline 11.57 & 9.75 & 9.66 & 10.77 & 13.04 \\
\hline 12.12 & 10.21 & 10.13 & 11.28 & 13.72 \\
\hline 12.65 & 10.68 & 10.58 & 11.79 & 14.38 \\
\hline 13.17 & 11.13 & 11.03 & 12.27 & 15.02 \\
\hline 13.68 & 11.57 & 11.47 & 12.75 & 15.66 \\
\hline 14.17 & 12.01 & 11.90 & 13.22 & 16.28 \\
\hline 14.65 & 12.44 & 12.33 & 13.68 & 16.88 \\
\hline 15.12 & 12.86 & 12.74 & 14.14 & 17.47 \\
\hline 15.57 & 13.27 & 13.15 & 14.59 & 18.05 \\
\hline 16.02 & 13.66 & 13.55 & 15.03 & 18.61 \\
\hline 16.45 & 14.05 & 13.93 & 15.47 & 19.16 \\
\hline 16.87 & 14.43 & 14.30 & 15.89 & 19.70 \\
\hline 17.28 & 14.79 & 14.67 & 16.30 & 20.22 \\
\hline 17.67 & 15.15 & 15.02 & 16.71 & 20.73 \\
\hline 18.06 & 15.49 & 15.37 & 17.11 & 21.23 \\
\hline
\end{tabular}

\begin{tabular}{|c|c|c|c|c|}
\hline \multicolumn{5}{|c|}{ INDUSTRIAL } \\
\hline Elec & Dist & Resid & NtGas & Coal \\
\hline & & & & $\ldots$ \\
\hline 0.98 & 0.91 & 1.00 & 0.97 & 0.98 \\
\hline 1.92 & 1.74 & 1.95 & 1.88 & 1.93 \\
\hline 2.82 & 2.53 & 2.80 & 2.71 & 2.86 \\
\hline 3.67 & 3.26 & 3.59 & 3.49 & 3.76 \\
\hline 4.48 & 3.93 & 4.31 & 4.23 & 4.63 \\
\hline 5.26 & 4.59 & 4.99 & 4.92 & 5.47 \\
\hline 6.01 & 5.20 & 5.64 & 5.59 & 6.29 \\
\hline 6.75 & 5.78 & 6.27 & 6.23 & 7.09 \\
\hline 7.47 & 6.37 & 6.88 & 6.87 & 7.87 \\
\hline 8.18 & 6.94 & 7.49 & 7.50 & 8.64 \\
\hline 8.74 & 7.39 & 7.95 & 7.97 & 9.24 \\
\hline 9.38 & 7.93 & 8.52 & 8.52 & 9.95 \\
\hline 10.01 & 8.46 & 9.07 & 9.06 & 10.66 \\
\hline 10.62 & 8.97 & 9.62 & 9.58 & 11.36 \\
\hline 11.22 & 9.47 & 10.16 & 10.10 & 12.05 \\
\hline 11.80 & 9.96 & 10.69 & 10.62 & 12.73 \\
\hline 12.37 & 10.44 & 11.21 & 11.13 & 13.39 \\
\hline 12.92 & 10.91 & 11.72 & 11.63 & 14.04 \\
\hline 13.45 & 11.37 & 12.22 & 12.12 & 14.68 \\
\hline 13.98 & 11.82 & 12.71 & 12.60 & 15.30 \\
\hline 14.48 & 12.27 & 13.19 & 13.07 & 15.91 \\
\hline 14.98 & 12.71 & 13.66 & 13.54 & 16.51 \\
\hline 15.47 & 13.13 & 14.12 & 14.00 & 17.10 \\
\hline 15.94 & 13.55 & 14.57 & 14.45 & 17.67 \\
\hline 16.40 & 13.96 & 15.00 & 14.89 & 18.23 \\
\hline 16.85 & 14.35 & 15.42 & 15.33 & 18.77 \\
\hline 17.29 & 14.73 & 15.83 & 15.76 & 19.30 \\
\hline 17.72 & 15.11 & 16.23 & 16.18 & 19.82 \\
\hline 18.13 & 15.47 & 16.62 & 16.59 & 20.32 \\
\hline 18.54 & 15.82 & 17.00 & 17.00 & 20.81 \\
\hline
\end{tabular}

Gasln

0.93

1.81

2.63

3.40

4.13

4.83
5.49

6.14

6.76

7.38

7.85

8.42

8.98

9.53

10.06

10.58

11.09

11.59

12.08

12.55

13.01

13.46

13.90

14.33

14.75

15.16

15.55

15.94

$\begin{array}{llll}17.47 & 14.49 & 18.09 & 16.49 \\ 17.90 & 14.86 & 18.53 & 16.92\end{array}$

$\begin{array}{llll}18.32 & 15.22 & 18.96 & 17.35\end{array}$

$\begin{array}{lllll}18.06 & 15.49 & 15.37 & 17.11 & 21.23\end{array}$

$\begin{array}{lllll}18.54 & 15.82 & 17.00 & 17.00 & 20.81\end{array}$

16.31

${ }^{\mathrm{a} O M B}$ discount rate as of February 2007.

See p. 20 for instructions on use; page xiii for abbreviations. 
Table Bb-5. OMB UPV* Discount Factors adjusted for fuel price escalation, by end-use sector and fuel type.

Discount Rate $=2.7 \%$ (years 1 to 10 ) and $3.0 \%$ (years 11 to 30 ), (OMB Circular A-94 $4^{\mathrm{a}}$ )

United States Average

RESIDENTIAL

\begin{tabular}{|c|c|c|c|}
\hline Elec & ist & LPG & NtGas \\
\hline & & & \\
\hline ๑. 97 & $\odot .96$ & 1.01 & ๑. $\mathrm{S}$ \\
\hline .91 & 1.84 & & \\
\hline 1 & 2.65 & 2.90 & \\
\hline & 3.3 & 3.79 & \\
\hline & 4.07 & 4.65 & 4.2 \\
\hline 33 & 4.70 & 5.2 & \\
\hline & 5.3 & 6.2 & \\
\hline & 5.8 & 7.6 & \\
\hline & & 7.82 & \\
\hline 35 & 7.02 & 8.57 & \\
\hline & 7.46 & 9.15 & \\
\hline & & & \\
\hline & & & \\
\hline & .0 & 5 & \\
\hline & .5 & & \\
\hline & & & \\
\hline 68 & .45 & 3.00 & 1. \\
\hline & & 3.5 & \\
\hline & & 5 & 12. \\
\hline & & 4. & 13. \\
\hline & 3 & 5.24 & \\
\hline & & & \\
\hline & & & \\
\hline & 7 & 16.7 & \\
\hline & 3.86 & 17.26 & \\
\hline & & & \\
\hline & & 19 & \\
\hline & & & \\
\hline & & & \\
\hline & & & \\
\hline
\end{tabular}

COMMERCIAL

\begin{tabular}{|c|c|c|c|c|}
\hline Elec & Dist & Resid & NtGas & Coal \\
\hline & - - - - & - - - - & - & \\
\hline 0.97 & 0.94 & 0.91 & 0.94 & 0.98 \\
\hline 1.90 & 1.80 & 1.78 & 1.82 & 1.93 \\
\hline 2.79 & 2.59 & 2.61 & 2.64 & 2.87 \\
\hline 3.64 & 3.31 & 3.39 & 3.41 & 3.78 \\
\hline 4.46 & 3.97 & 4.11 & 4.14 & 4.66 \\
\hline 5.24 & 4.59 & 4.74 & 4.84 & 5.51 \\
\hline 6.00 & 5.18 & 5.36 & 5.51 & 6.32 \\
\hline 6.74 & 5.75 & 5.98 & 6.16 & 7.11 \\
\hline 7.47 & 6.31 & 6.59 & 6.80 & 7.88 \\
\hline 8.18 & 6.86 & 7.19 & 7.43 & 8.63 \\
\hline 8.74 & 7.29 & 7.66 & 7.92 & 9.19 \\
\hline 9.40 & 7.81 & 8.23 & 8.49 & 9.86 \\
\hline 10.04 & 8.32 & 8.81 & 9.05 & 10.52 \\
\hline 10.66 & 8.82 & 9.38 & 9.59 & 11.16 \\
\hline 11.27 & 9.31 & 9.94 & 10.12 & 11.79 \\
\hline 11.86 & 9.79 & 10.48 & 10.64 & 12.39 \\
\hline 12.43 & 10.25 & 11.01 & 11.16 & 12.99 \\
\hline 12.99 & 10.71 & 11.52 & 11.66 & 13.57 \\
\hline 13.53 & 11.16 & 12.01 & 12.14 & 14.13 \\
\hline 14.06 & 11.60 & 12.50 & 12.62 & 14.69 \\
\hline 14.57 & 12.03 & 12.98 & 13.08 & 15.23 \\
\hline 15.07 & 12.45 & 13.43 & 13.54 & 15.76 \\
\hline 15.55 & 12.87 & 13.87 & 13.99 & 16.27 \\
\hline 16.02 & 13.27 & 14.30 & 14.42 & 16.77 \\
\hline 16.48 & 13.67 & 14.72 & 14.85 & 17.27 \\
\hline 16.92 & 14.05 & 15.13 & 15.27 & 17.74 \\
\hline 17.36 & 14.42 & 15.52 & 15.68 & 18.21 \\
\hline 17.78 & & 15.91 & 16.08 & 18.66 \\
\hline 18.19 & 15.14 & 16.28 & 16.48 & \\
\hline & & & 16.86 & 19.54 \\
\hline
\end{tabular}

INDUSTRIAL

Elec Dist Resid NtGas Coal O. $\begin{array}{lllll}0.98 & 0.92 & 0.99 & 0.96 & 0.98\end{array}$

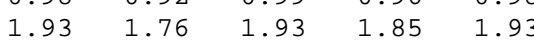
$\begin{array}{lllll}2.83 & 2.55 & 2.79 & 2.66 & 2.87\end{array}$ $\begin{array}{lllll}3.67 & 3.29 & 3.60 & 3.41 & 3.77\end{array}$ $\begin{array}{lllll}4.46 & 3.97 & 4.36 & 4.11 & 4.65\end{array}$ $\begin{array}{lllll}5.22 & 4.62 & 5.06 & 4.77 & 5.49\end{array}$ $\begin{array}{lllll}5.96 & 5.24 & 5.72 & 5.41 & 6.32\end{array}$ $\begin{array}{lllll}6.67 & 5.84 & 6.38 & 6.03 & 7.11\end{array}$ $\begin{array}{lllll}7.37 & 6.43 & 7.02 & 6.64 & 7.89\end{array}$ $\begin{array}{lllll}8.06 & 7.01 & 7.65 & 7.25 & 8.64\end{array}$ $\begin{array}{lllll}8.60 & 7.46 & 8.14 & 7.72 & 9.21\end{array}$ $\begin{array}{lllll}9.24 & 8.01 & 8.74 & 8.27 & 9.90\end{array}$ $\begin{array}{lllll}9.86 & 8.54 & 9.33 & 8.82 & 10.56\end{array}$ $\begin{array}{lllll}10.47 & 9.06 & 9.91 & 9.35 & 11.21\end{array}$ $\begin{array}{lllll}11.06 & 9.58 & 10.49 & 9.86 & 11.85\end{array}$ $\begin{array}{lllll}11.63 & 10.07 & 11.04 & 10.38 & 12.47\end{array}$ $\begin{array}{lllll}12.20 & 10.56 & 11.58 & 10.89 & 13.07\end{array}$ $\begin{array}{lllll}12.74 & 11.04 & 12.11 & 11.38 & 13.66\end{array}$ $\begin{array}{lllll}12.74 & 11.04 & 12.11 & 11.38 & 13.66 \\ 13.27 & 11.51 & 12.63 & 11.86 & 14.23\end{array}$ $\begin{array}{lllll}13.27 & 11.51 & 12.63 & 11.86 & 14.23 \\ 13.79 & 11.97 & 13.13 & 12.34 & 14.79\end{array}$ $\begin{array}{llllll}13.79 & 11.97 & 13.13 & 12.34 & 14.79\end{array}$ $\begin{array}{lllll}14.28 & 12.42 & 13.63 & 12.80 & 15.33 \\ 14.77 & 12.86 & 14.11 & 13.26 & 15.83\end{array}$ $\begin{array}{lllll}14.77 & 12.86 & 14.11 & 13.26 & 15.86\end{array}$ $\begin{array}{lllll}15.25 & 13.30 & 14.57 & 13.71 & 16.38\end{array}$ $\begin{array}{llllll}15.71 & 13.72 & 15.03 & 14.15 & 16.89\end{array}$ $\begin{array}{lllll}16.16 & 14.14 & 15.48 & 14.59 & 17.38\end{array}$ $\begin{array}{llllll}16.59 & 14.54 & 15.91 & 15.01 & 17.86\end{array}$ $\begin{array}{lllll}17.02 & 14.93 & 16.33 & 15.43 & 18.33\end{array}$ $\begin{array}{lllll}17.43 & 15.31 & 16.73 & 15.84 & 18.79\end{array}$ $\begin{array}{lllll}17.83 & 15.67 & 17.13 & 16.24 & 19.23\end{array}$ $\begin{array}{lllll}18.22 & 16.03 & 17.52 & 16.64 & 19.67\end{array}$

$\begin{array}{rr}\text { TRANSPORT } & \\ \text { Gasln } & \mathrm{N} \\ --.- & - \\ 0.93 & 1 \\ 1.78 & 2 \\ 2.58 & 3 \\ 3.32 & 4 \\ 4.03 & 5 \\ 4.70 & 6 \\ 5.34 & 7 \\ 5.96 & 8 \\ 6.56 & 9 \\ 7.16 & 10 \\ 7.61 & 11 \\ 8.16 & 12 \\ 8.70 & 13 \\ 9.23 & 14 \\ 9.75 & 15 \\ 10.25 & 16 \\ 10.74 & 17 \\ 11.22 & 18 \\ 11.69 & 19 \\ 12.15 & 20 \\ 12.59 & 21 \\ 13.03 & 22 \\ 13.45 & 23 \\ 13.87 & 24 \\ 14.27 & 25 \\ 14.67 & 26 \\ 15.05 & 27 \\ 15.42 & 28 \\ 15.78 & 29 \\ 16.13 & 30 \\ & \end{array}$

${ }^{a}$ OMB discount rate as of February 2007

See p. 20 for instructions on use; page xiii for abbreviations. 


\section{Projected Average Fuel Price Indices and Escalation Rates (Real)}

Tables Ca-1 through Ca-5 present projected fuel price indices for the four Census regions and for the United States. These indices, when multiplied by annual energy costs computed at basedate prices (i.e., as of April 1, 2007), provide estimates of future-year costs (also as of April 1) in constant base-date dollars. Constant-dollar cost estimates are needed when discounting is performed with a real discount rate (i.e., a rate that does not include general price inflation).

These indices were used in the calculation of the UPV* factors for energy prices in the Ba and $\mathrm{Bb}$ tables in this publication. While they are based on April 1 energy prices to maintain consistency in the computation of these UPV* factors, the level of precision implied here is not required for most LCC analyses. That is, the analyst need not calibrate base-year energy prices precisely to April 1, 2007 levels to use these indices (or the corresponding UPV* factors); instead, the analyst should use current price levels as of the base-date of the LCC analysis, regardless of the time of the year that the study is undertaken.

\section{Example of How to Use the Indices:}

To estimate the price of industrial coal in 2010 in Connecticut (in constant 2007 dollars), go to Table Ca-1, find the year 2010 index for industrial coal (1.02), and multiply by the price for industrial coal in Connecticut in 2007.

For further explanation of how to use these tables, see NIST Handbook 135.

Tables $\boldsymbol{C b}-\mathbf{1}$ through $\boldsymbol{C b}-\mathbf{5}$ present the projected average fuel price escalation rates (percentage change compounded annually) for selected periods from 2007 to 2037 for the four Census regions and for the overall United States. Note that these are real rates exclusive of general price inflation. Their use results in prices expressed in constant dollars.

The average fuel escalation rates consolidate the information provided by the indices in the Ca tables so that trends in projected price changes can be seen at a glance. They are provided primarily to accommodate computer programs (such as BLCC) which require price escalation rates as inputs.

Unless there is a compelling reason to use escalation rates, it is recommended that you use the indices in the Ca tables when you need estimates of future-year energy prices, since the indices include year-to-year information rather than averages over a number of years.

\section{Example of How to Use the Escalation Rates:}

To estimate the unit price of residential natural gas at the end of $2017\left(\mathrm{p}_{17}\right)$ in Wyoming using the DOE energy price escalation rates, go to Table Cb-4 and find the 2007 to 2012 and the 2012 to 2017 escalation rates for residential natural gas ( $-2.5 \%$ for 5 years and $-0.1 \%$ for 5 years, respectively). Enter these values and the unit price of residential natural gas in Wyoming in 2007 $\left(\mathrm{p}_{07}\right)$ into the following formula. Then solve for the 2017 energy price (stated in 2007 dollars): 


$$
\begin{aligned}
p_{y} & =p_{0} \times \prod_{i=1}^{N}\left(1+e_{i}\right)^{k_{i}} \\
p_{17} & =p_{07} \times\left(1+e_{1}\right)^{k_{1}} \times\left(1+e_{2}\right)^{k_{2}} \\
& =p_{07} \times(1-0.025)^{5} \times(1-0.001)^{5} \\
& =p_{07} \times 0.8811 \times 0.9950 \\
& =p_{07} \times 0.877
\end{aligned}
$$

where $\mathrm{p}_{\mathrm{y}}=$ price at end of year $\mathrm{y}$;

$\mathrm{p}_{0}=$ unit price at base date;

$\mathrm{e}_{\mathrm{i}}=$ annual compound escalation rate for period i from the Cb tables (in decimal form); and

$\mathrm{k}_{\mathrm{i}}=$ number of years over which escalation rate $\mathrm{e}_{\mathrm{i}}$ occurs.

Note that the compounded escalation rate factor $(0.877)$ corresponds to the fuel price index in region 4, residential natural gas, for the year 2017 in table Ca-4 (0.88).

The data in the $\mathrm{Ca}$ and $\mathrm{Cb}$ tables on the following pages are reported for the four Census regions and the U.S. average. Figure B-1 on page 13 presents a map showing the states corresponding to the four Census regions. The Census regions do not include American Samoa, Canal Zone, Guam, Puerto Rico, Trust Territory of the Pacific Islands, or the Virgin Islands. Analysts of federal projects in these areas should use data which are "reasonable under the circumstances," and may refer to the tables with U.S. average data for guidance. 
Table Ca-1. Projected fuel price indices (excluding general inflation), by end-use sector and fuel type.

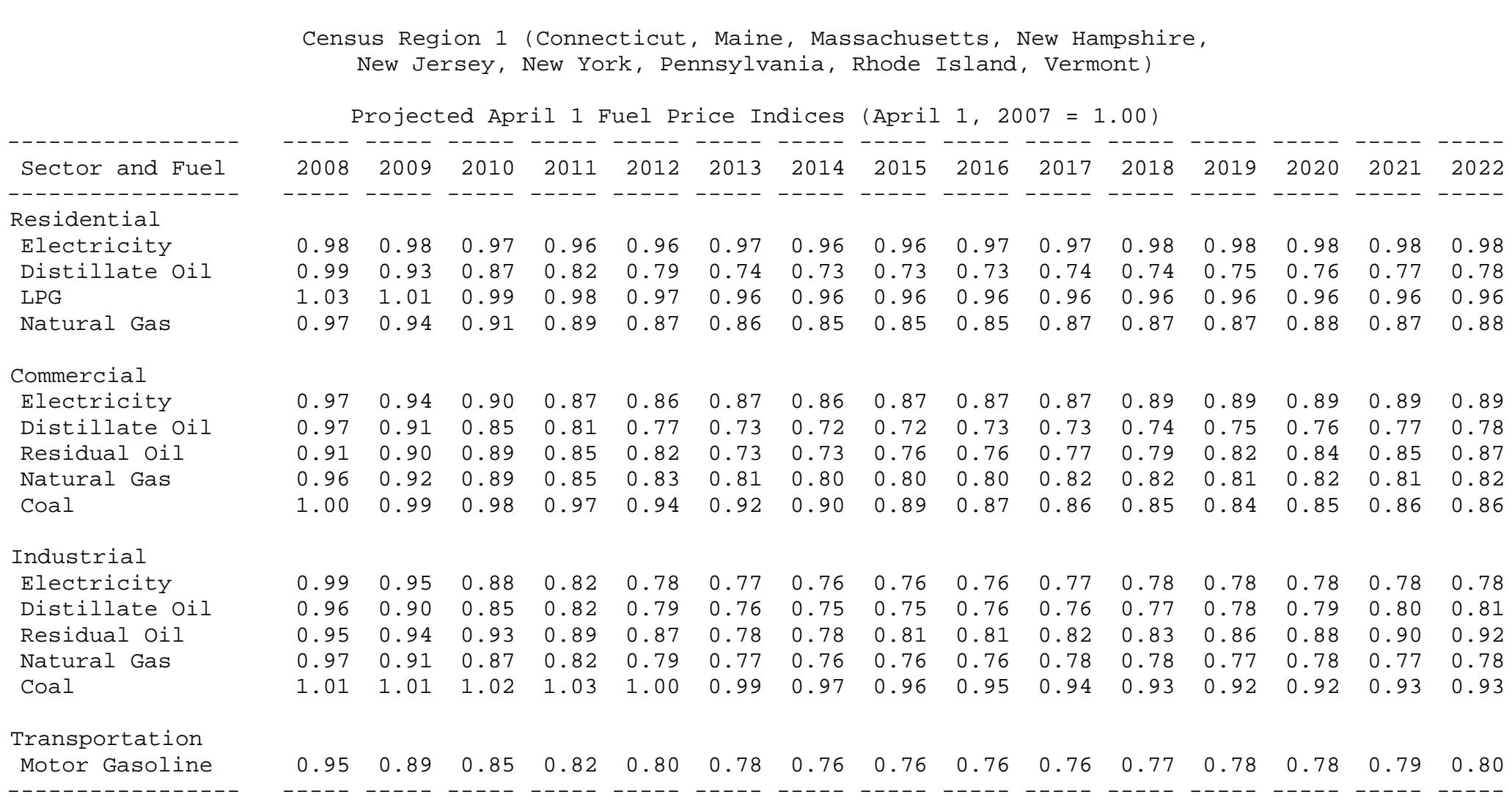


Table Ca-1, continued. Projected fuel price indices (excluding general inflation), by end-use sector and fuel type.

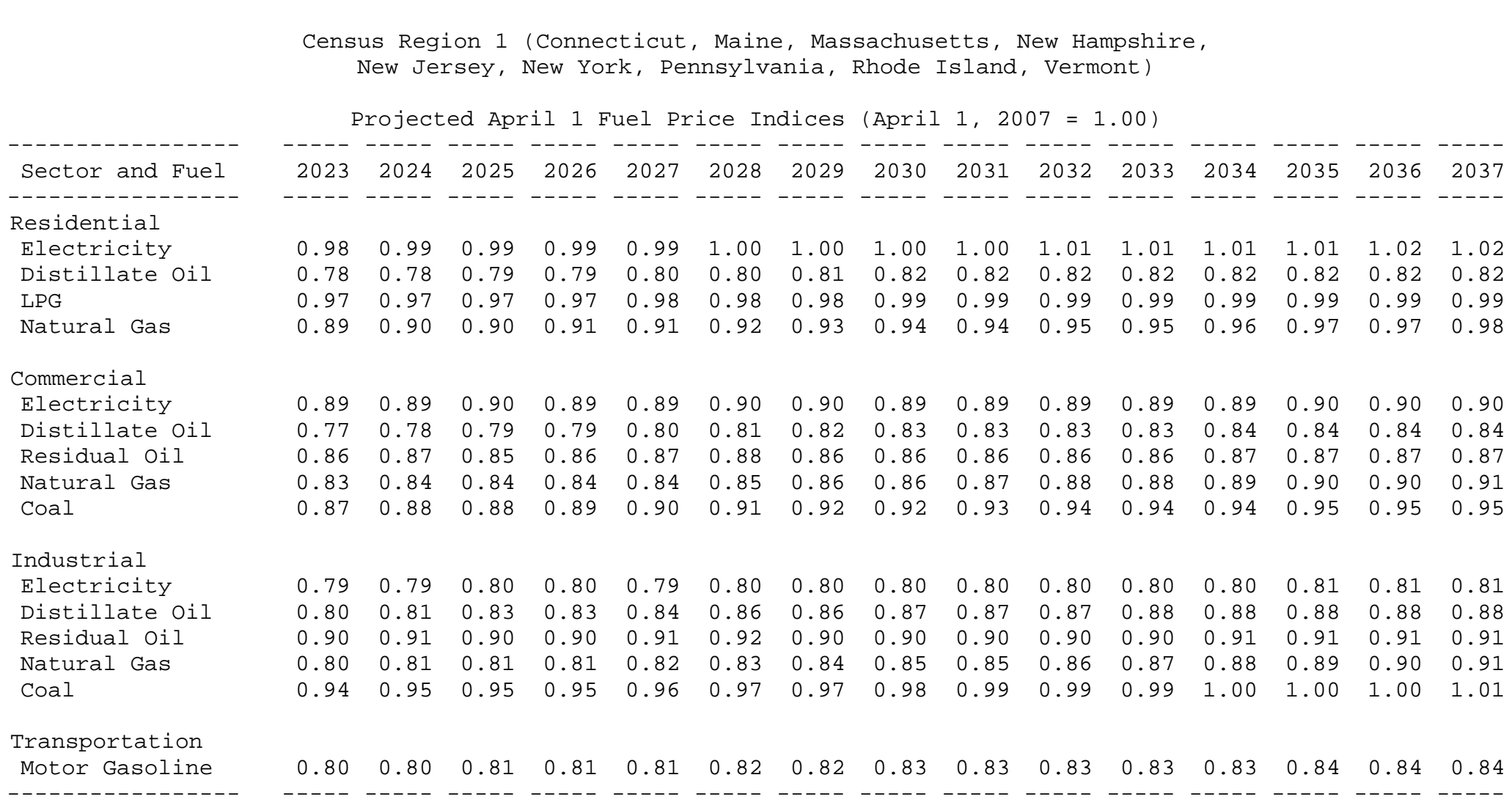


Table Ca-2. Projected fuel price indices (excluding general inflation), by end-use sector and fuel type.

Census Region 2 (Illinois, Indiana, Iowa, Kansas, Michigan, Minnesota,

Missouri, Nebraska, North Dakota, Ohio, South Dakota, Wisconsin)

Projected April 1 Fuel Price Indices (April 1, $2007=1.00$ )

Sector and Fuel

Residential

Electricity

Distillate 0il

LPG

Natural Gas

Commercial

Electricity

Distillate 0il

Residual oil

Natural Gas

Coal

Industria

Electricity

Distillate 0il

Residual 0il

Natural Gas

Coal

Transportation

Motor Gasoline

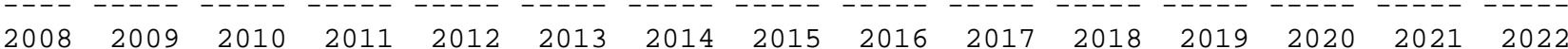

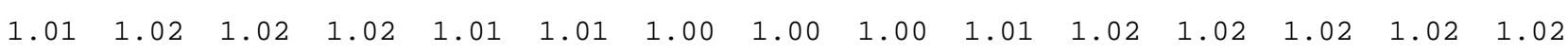

$\begin{array}{lllllllllllllll}0.98 & 0.93 & 0.86 & 0.80 & 0.74 & 0.70 & 0.68 & 0.68 & 0.68 & 0.69 & 0.70 & 0.71 & 0.71 & 0.72 & 0.73\end{array}$

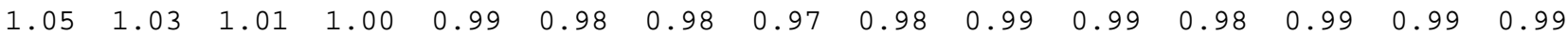

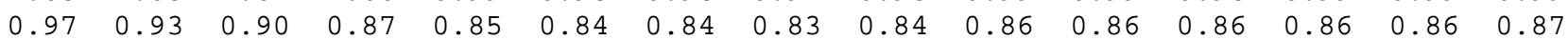

\begin{tabular}{|c|c|c|c|c|c|c|c|c|c|c|c|c|c|c|}
\hline 1.01 & 1.02 & 1.02 & 1.02 & 1.00 & 0.99 & $\odot .98$ & $\odot .98$ & $\odot .98$ & $\odot .99$ & 1.00 & 1.01 & 1.02 & 1.02 & 1.02 \\
\hline 0.96 & 0.89 & 0.84 & 0.79 & 0.74 & 0.71 & 0.69 & 0.68 & $\odot .69$ & 0.70 & $\odot .70$ & 0.72 & 0.72 & 0.73 & 0.74 \\
\hline 1.09 & 1.05 & 1.00 & 0.96 & $\odot .92$ & 0.90 & 0.88 & $\odot .88$ & $\odot .88$ & 0.89 & $\odot .89$ & 0.91 & 0.92 & 0.93 & 0.94 \\
\hline$\odot .97$ & 0.92 & $\odot .89$ & 0.86 & $\odot .83$ & ๑.81 & $\odot .81$ & $\odot .80$ & ๑.81 & 0.83 & 0.83 & ๑. 82 & 0.83 & 0.83 & $\odot .83$ \\
\hline 1.01 & 1.02 & 1.05 & 1.06 & 1.06 & 1.06 & 1.06 & 1.05 & 1.04 & 1.03 & 1.02 & 1.02 & 1.02 & 1.02 & 1.02 \\
\hline
\end{tabular}

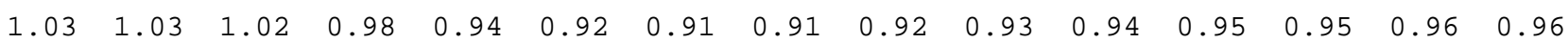

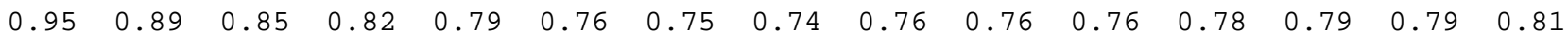

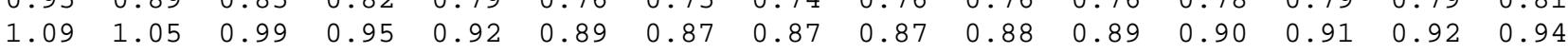

$\begin{array}{lllllllllllllll}1.00 & 0.95 & 0.91 & 0.86 & 0.84 & 0.81 & 0.81 & 0.80 & 0.81 & 0.83 & 0.83 & 0.82 & 0.83 & 0.83 & 0.84\end{array}$

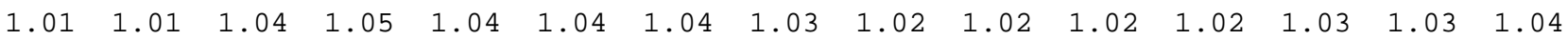

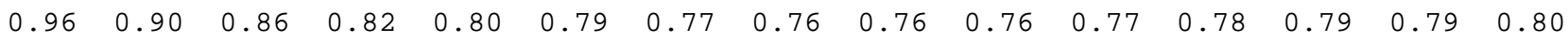


Table Ca-2, continued. Projected fuel price indices (excluding general inflation), by end-use sector and fuel type.

Census Region 2 (Illinois, Indiana, Iowa, Kansas, Michigan, Minnesota,

Missouri, Nebraska, North Dakota, Ohio, South Dakota, Wisconsin)

Projected April 1 Fuel Price Indices (April 1, $2007=1.00$ )

Sector and Fuel

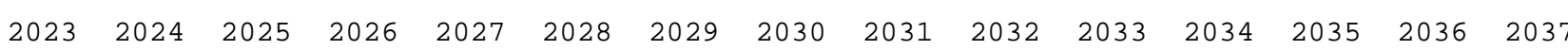

-.-.-

Residential

Electricity

Distillate oil

LPG

Natural Gas

Commercial

Electricity

Distillate oil

Residual oil

Natural Gas

Coal

Industrial

Electricity

Distillate 0il

Residual oil

Natural Gas

coal

$\begin{array}{llllllllllllllll}1.02 & 1.02 & 1.02 & 1.02 & 1.02 & 1.02 & 1.02 & 1.02 & 1.02 & 1.02 & 1.02 & 1.02 & 1.02 & 1.02 & 1.02 \\ 0.73 & 0.73 & 0.74 & 0.74 & 0.75 & 0.75 & 0.76 & 0.77 & 0.77 & 0.77 & 0.77 & 0.77 & 0.77 & 0.77 & 0.77 \\ 1.00 & 1.00 & 1.0 \odot & 1.00 & 1.01 & 1.01 & 1.02 & 1.02 & 1.02 & 1.02 & 1.02 & 1.02 & 1.02 & 1.03 & 1.03 \\ 0.89 & 0.90 & 0.90 & 0.90 & 0.91 & 0.92 & 0.93 & 0.94 & 0.95 & 0.95 & 0.96 & 0.97 & 0.97 & 0.98 & 0.99\end{array}$

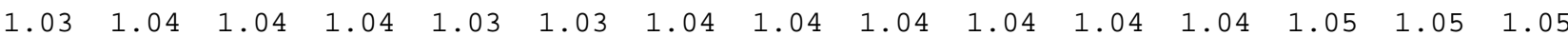

$\begin{array}{lllllllllllllll}0.74 & 0.74 & 0.75 & 0.76 & 0.77 & 0.78 & 0.78 & 0.79 & 0.80 & 0.80 & 0.80 & 0.80 & 0.80 & 0.80 & 0.80\end{array}$

$\begin{array}{lllllllllllllll}0.94 & 0.95 & 0.95 & 0.96 & 0.96 & 0.97 & 0.98 & 0.98 & 0.99 & 0.99 & 0.99 & 0.99 & 0.99 & 0.99 & 0.99\end{array}$

$\begin{array}{lllllllllllllll}0.84 & 0.85 & 0.86 & 0.86 & 0.86 & 0.87 & 0.88 & 0.89 & 0.89 & 0.90 & 0.91 & 0.92 & 0.92 & 0.93 & 0.94\end{array}$

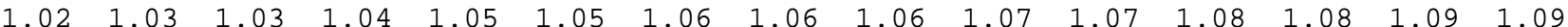

Transportation

Motor Gasoline

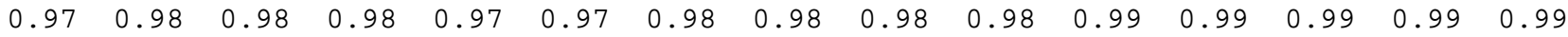

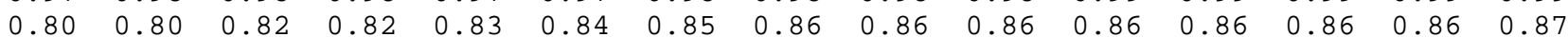

$\begin{array}{lllllllllllllll}0.93 & 0.94 & 0.94 & 0.95 & 0.96 & 0.96 & 0.97 & 0.98 & 0.98 & 0.98 & 0.98 & 0.99 & 0.99 & 0.99 & 0.99\end{array}$

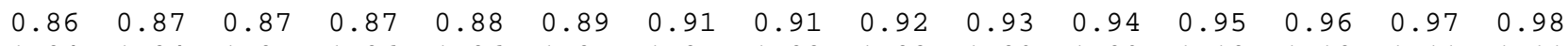

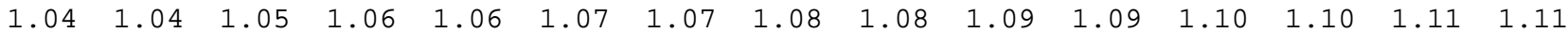

- . - - - . - . - -

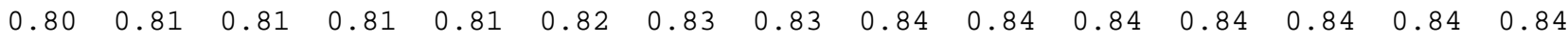




\section{Table Ca-3. Projected fuel price indices (excluding general inflation), by end-use sector and fuel type.}

Census Region 3 (Alabama, Arkansas, Delaware, District of Columbia, Florida, Georgia, Kentucky, Louisiana, Maryland, Mississippi, North Carolina, Oklahoma, South Carolina,

Tennessee, Texas, Virginia, West Virginia)

Projected April 1 Fuel Price Indices (April 1, $2007=1.00$ )

\begin{tabular}{|c|c|c|c|c|c|c|c|c|c|c|c|c|c|c|c|}
\hline Sector and Fuel & $20 \odot 8$ & 2009 & 2010 & 2011 & 2012 & 2013 & 2014 & 2015 & 2016 & 2017 & 2018 & 2019 & 2020 & 2021 & 2022 \\
\hline Residential & $-\ldots$ & $-\ldots$ & $-\ldots$ & ---- & $-\ldots$ & $-\ldots$ & $-\ldots$ & $-\ldots$ & $-\ldots$ & $-\ldots$ & $-\ldots$ & $-\ldots$ & $--_{-}-$ & 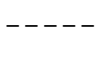 & \\
\hline Electricity & $\odot .99$ & $\odot .98$ & 0.97 & 0.96 & 0.94 & 0.93 & 0.93 & 0.93 & 0.93 & 0.93 & 0.94 & 0.94 & 0.94 & $\odot .94$ & $0 . \mathrm{s}$ \\
\hline Distillate oil & 0.99 & 0.93 & 0.88 & 0.83 & 0.79 & 0.74 & 0.73 & 0.73 & 0.73 & 0.74 & 0.75 & 0.75 & 0.76 & 0.77 & \\
\hline LPG & 1.03 & 1.01 & $1.0 \odot$ & $\odot .98$ & 0.97 & 0.96 & $\odot .96$ & $\odot .96$ & $\odot .96$ & $\odot .97$ & $\odot .97$ & $\odot .97$ & $\odot .97$ & $\odot .97$ & ๑. \\
\hline Natural Gas & 0.97 & 0.94 & $\odot .91$ & 0.88 & 0.86 & 0.85 & 0.85 & 0.85 & 0.86 & 0.87 & 0.88 & 0.88 & 0.89 & 0.89 & 0.8 \\
\hline \multicolumn{16}{|l|}{ Commercial } \\
\hline Electricity & $1.0 \odot$ & $\odot .99$ & 0.98 & 0.96 & $\odot .95$ & $\odot .94$ & 0.93 & 0.93 & 0.94 & 0.95 & 0.96 & 0.96 & 0.96 & 0.96 & 0.9 \\
\hline Distillate oil & $\odot .97$ & $\odot .91$ & $\odot .85$ & $\odot .80$ & 0.75 & 0.72 & $\odot .70$ & $\odot .70$ & 0.71 & $\odot .72$ & $\odot .72$ & 0.73 & 0.74 & 0.75 & 0.7 \\
\hline Residual oil & 1.02 & $\odot .97$ & $\odot .91$ & 0.86 & 0.82 & 0.79 & 0.77 & 0.77 & 0.77 & 0.78 & 0.79 & 0.80 & 0.81 & 0.83 & $\odot .8$ \\
\hline Natural Gas & 0.97 & 0.93 & 0.89 & 0.85 & 0.83 & 0.81 & 0.81 & 0.80 & 0.81 & $\odot .82$ & 0.83 & 0.83 & 0.83 & 0.83 & 0.8 \\
\hline Coal & 1.00 & 1.00 & 0.99 & 0.97 & 0.95 & 0.93 & 0.93 & 0.94 & 0.94 & 0.93 & 0.92 & 0.90 & 0.90 & 0.90 & 0.9 \\
\hline \multicolumn{16}{|l|}{ Industrial } \\
\hline Electricity & 1.01 & 1.00 & $\odot .98$ & 0.95 & 0.92 & 0.91 & 0.90 & 0.90 & 0.91 & 0.92 & 0.93 & 0.93 & 0.94 & 0.94 & \\
\hline Distillate 0il & $\odot .96$ & 0.89 & 0.85 & $\odot .82$ & 0.78 & $\odot .76$ & 0.74 & $\odot .74$ & 0.75 & 0.76 & 0.76 & 0.78 & 0.79 & 0.79 & \\
\hline Residual 0il & 1.04 & 1.00 & $\odot .94$ & $\odot .90$ & $\odot .86$ & 0.83 & 0.81 & 0.81 & 0.81 & 0.82 & 0.83 & 0.84 & 0.85 & 0.87 & $0 . \varepsilon$ \\
\hline Natural Gas & 0.99 & $\odot .92$ & $\odot .86$ & $\odot .80$ & $\odot .76$ & 0.74 & 0.74 & 0.74 & 0.75 & 0.77 & 0.78 & 0.78 & 0.78 & 0.78 & 0.7 \\
\hline Coal & 1.00 & 1.00 & $\odot .99$ & $\odot .98$ & $\odot .97$ & 0.96 & 0.96 & 0.95 & 0.95 & 0.95 & 0.94 & 0.94 & 0.94 & 0.94 & $0 . \mathrm{s}$ \\
\hline \multicolumn{16}{|l|}{ Transportation } \\
\hline Motor Gasoline & 0.95 & 0.90 & 0.85 & 0.82 & $\odot .80$ & 0.78 & 0.76 & 0.75 & $\odot .76$ & 0.76 & 0.77 & 0.78 & 0.78 & 0.79 & $0 . \varepsilon$ \\
\hline & & & & & & & & & & & & & & & \\
\hline
\end{tabular}


Table Ca-3, continued. Projected fuel price indices (excluding general inflation), by end-use sector and fuel type.

Census Region 3 (Alabama, Arkansas, Delaware, District of Columbia, Florida, Georgia, Kentucky, Louisiana, Maryland, Mississippi, North Carolina, Oklahoma, South Carolina,

Tennessee, Texas, Virginia, West Virginia)

\begin{tabular}{|c|c|c|c|c|c|c|c|c|c|c|c|c|c|c|}
\hline-- & --- & - - - - & - - - - & $---{ }_{-}^{-}$ & - - - - & $---{ }_{-}$ & 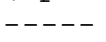 & - - - - & - - - - - & --- & - - - - & --- & - - - - & --- \\
\hline 2023 & 2024 & 2025 & 2026 & 2027 & 2028 & 2029 & 2030 & 2031 & 2032 & 2033 & 2034 & 2035 & 2036 & 2037 \\
\hline - - & - & 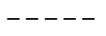 & - - - - & --- & - & --- & --- & 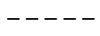 & --- & $---{ }_{-}^{-}$ & --- & --- & --- & --- \\
\hline 0.94 & $\odot .94$ & $\odot .95$ & $\odot .95$ & $\odot .95$ & 0.95 & $\odot .95$ & $\odot .95$ & $\odot .95$ & $\odot .95$ & $\odot .96$ & 0.96 & $\odot .96$ & $\odot .96$ & 0.96 \\
\hline 0.78 & 0.78 & 0.79 & $\odot .79$ & 0.80 & 0.81 & 0.81 & 0.82 & 0.82 & 0.82 & 0.82 & 0.82 & 0.82 & 0.82 & 0.83 \\
\hline 0.98 & $\odot .99$ & $\odot .99$ & $\odot .99$ & $\odot .99$ & 0.99 & 1.00 & 1.00 & $1.0 \odot$ & 1.00 & 1.00 & 1.00 & $1.0 \odot$ & 1.00 & 1.00 \\
\hline 0.91 & $\odot .92$ & $\odot .92$ & 0.92 & $\odot .93$ & 0.94 & $\odot .95$ & 0.95 & 0.96 & $\odot .96$ & 0.97 & 0.98 & $\odot .98$ & 0.99 & 1.00 \\
\hline$\odot .96$ & $\odot .97$ & $\odot .97$ & 0.97 & $\odot .97$ & 0.97 & $\odot .97$ & $\odot .97$ & 0.97 & $\odot .98$ & $\odot .98$ & 0.98 & $\odot .98$ & 0.98 & 0.98 \\
\hline$\odot .76$ & 0.76 & 0.78 & 0.78 & $\odot .79$ & 0.80 & 0.81 & 0.82 & 0.82 & 0.82 & 0.82 & 0.82 & 0.82 & 0.83 & 0.83 \\
\hline 0.84 & 0.85 & $\odot .86$ & $\odot .87$ & 0.87 & 0.88 & $\odot .89$ & $\odot .90$ & 0.90 & $\odot .9 \odot$ & $\odot .90$ & 0.90 & $\odot .91$ & 0.91 & 0.91 \\
\hline 0.85 & 0.86 & $\odot .86$ & $\odot .86$ & 0.86 & 0.87 & ๑.88 & 0.88 & 0.89 & 0.90 & 0.90 & 0.91 & 0.92 & 0.93 & 0.94 \\
\hline 0.90 & 0.90 & 0.90 & 0.90 & 0.90 & 0.91 & 0.91 & 0.92 & 0.92 & 0.92 & 0.93 & 0.93 & 0.94 & 0.94 & 0.94 \\
\hline 0.94 & 0.94 & 0.95 & 0.95 & 0.94 & 0.94 & 0.95 & 0.95 & 0.95 & $\odot .95$ & 0.95 & 0.95 & 0.95 & 0.96 & 0.96 \\
\hline$\odot .80$ & $\odot .80$ & 0.82 & 0.82 & 0.83 & 0.85 & 0.86 & 0.87 & 0.87 & 0.87 & 0.87 & 0.87 & 0.87 & 0.87 & 0.87 \\
\hline 0.87 & 0.88 & $\odot .89$ & $\odot .90$ & $\odot .90$ & 0.91 & 0.92 & 0.93 & 0.93 & $\odot .93$ & 0.93 & 0.93 & 0.94 & 0.94 & 0.94 \\
\hline 0.81 & 0.83 & $\odot .83$ & 0.83 & 0.84 & 0.85 & $\odot .86$ & 0.87 & 0.88 & $\odot .89$ & $\odot .90$ & 0.92 & 0.93 & 0.94 & 0.95 \\
\hline 0.94 & 0.94 & 0.95 & 0.95 & 0.95 & 0.95 & 0.96 & 0.96 & 0.97 & 0.97 & 0.97 & 0.98 & 0.98 & 0.98 & 0.99 \\
\hline$\odot .80$ & 0.80 & 0.81 & 0.81 & 0.81 & 0.82 & 0.83 & 0.83 & 0.84 & 0.84 & 0.84 & 0.84 & 0.84 & 0.84 & 0.84 \\
\hline & & & & & & & & & & & & & & \\
\hline
\end{tabular}


Table Ca-4. Projected fuel price indices (excluding general inflation), by end-use sector and fuel type.

Census Region 4 (Alaska, Arizona, California, Colorado, Hawaii,

Idaho, Montana, Nevada, New Mexico, Oregon, Utah, Washington, Wyoming)

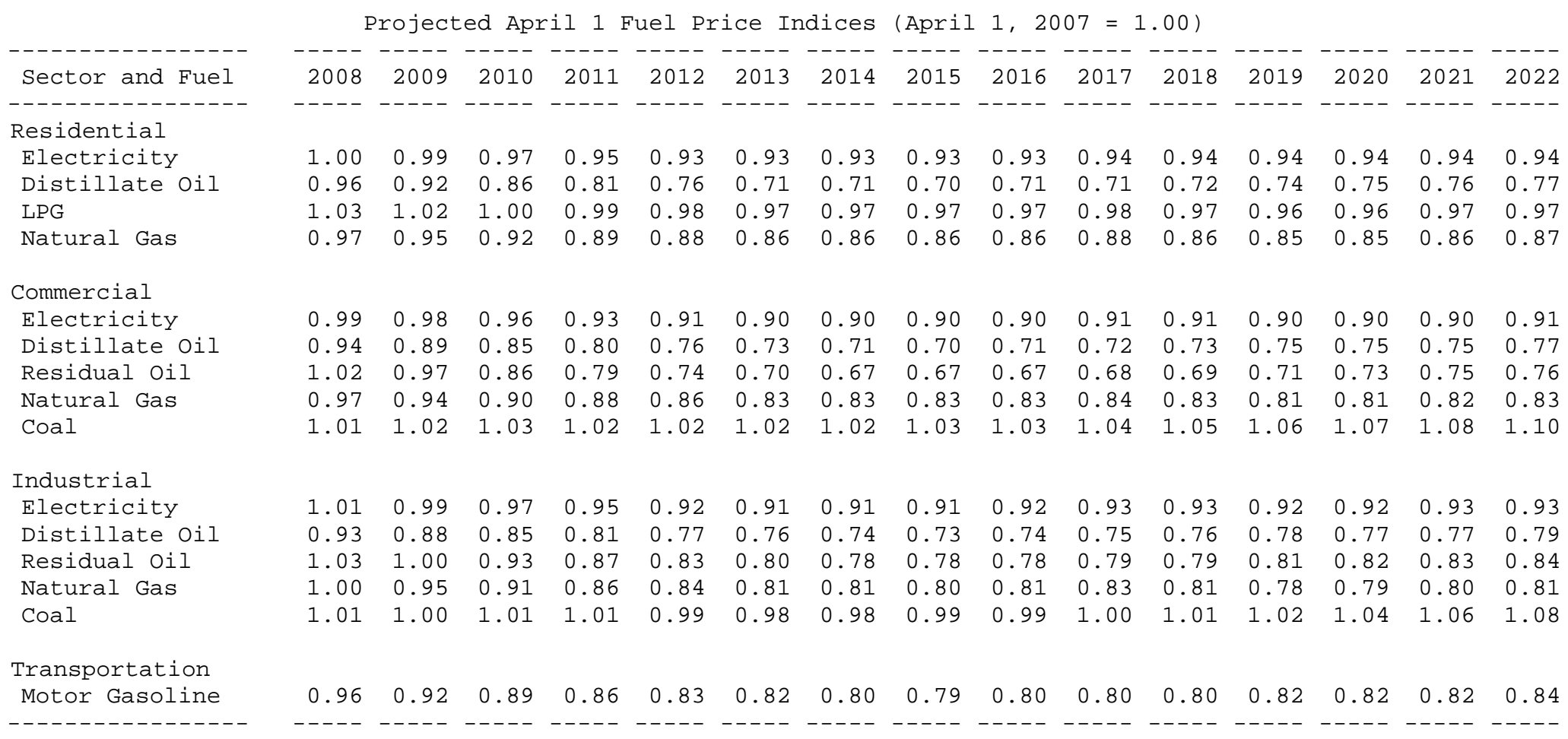


Table Ca-4, continued. Projected fuel price indices (excluding general inflation), by end-use sector and fuel type.

Census Region 4 (Alaska, Arizona, California, Colorado, Hawaii,

Idaho, Montana, Nevada, New Mexico, Oregon, Utah, Washington, Wyoming)

Projected April 1 Fuel Price Indices (April 1, $2007=1.00$ )

Sector and Fuel

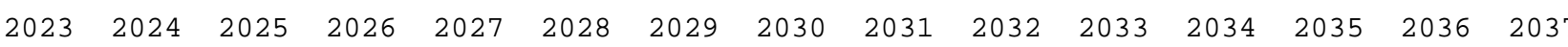

-....-

Residential

Electricity

Distillate oil

LPG

Natural Gas

Commercial

Electricity

Distillate oil

Residual oil

Natural Gas

Coal

Industrial

Electricity

Distillate 0il

Residual oil

Natural Gas

coal

$\begin{array}{lllllllllllllll}0.95 & 0.95 & 0.95 & 0.96 & 0.96 & 0.96 & 0.96 & 0.97 & 0.97 & 0.98 & 0.98 & 0.98 & 0.99 & 0.99 & 1.00 \\ 0.77 & 0.78 & 0.79 & 0.80 & 0.81 & 0.82 & 0.83 & 0.84 & 0.84 & 0.84 & 0.84 & 0.84 & 0.84 & 0.84 & 0.84 \\ 0.98 & 0.98 & 0.99 & 0.99 & 0.99 & 1.00 & 1.00 & 1.00 & 1.01 & 1.01 & 1.01 & 1.01 & 1.01 & 1.01 & 1.01 \\ 0.89 & 0.90 & 0.90 & 0.91 & 0.91 & 0.93 & 0.94 & 0.95 & 0.96 & 0.96 & 0.97 & 0.98 & 0.98 & 0.99 & 1.00\end{array}$

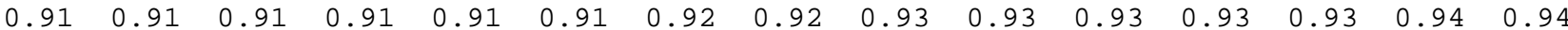

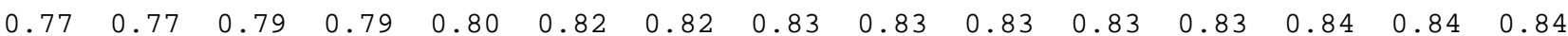

$\begin{array}{lllllllllllllll}0.75 & 0.77 & 0.77 & 0.79 & 0.80 & 0.80 & 0.81 & 0.82 & 0.83 & 0.83 & 0.83 & 0.83 & 0.83 & 0.84 & 0.84\end{array}$

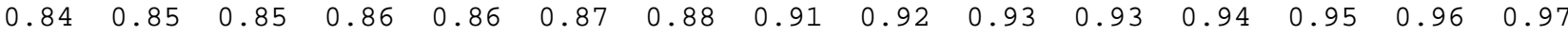

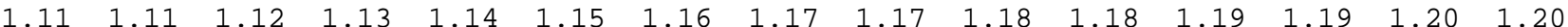

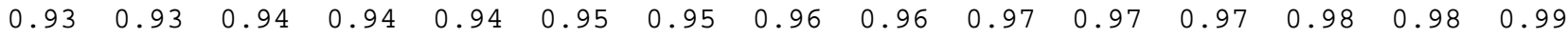

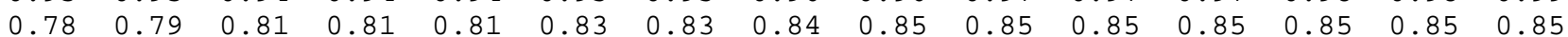

$\begin{array}{lllllllllllllll}0.85 & 0.86 & 0.86 & 0.88 & 0.89 & 0.89 & 0.91 & 0.91 & 0.91 & 0.91 & 0.91 & 0.91 & 0.91 & 0.91 & 0.92\end{array}$

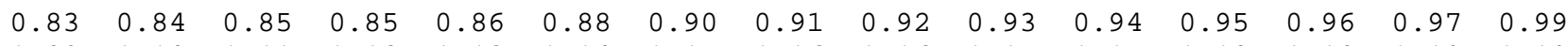

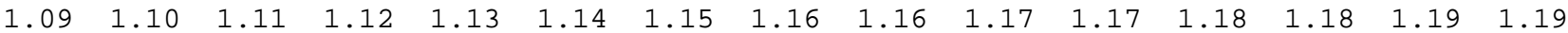

Transportation

Motor Gasoline

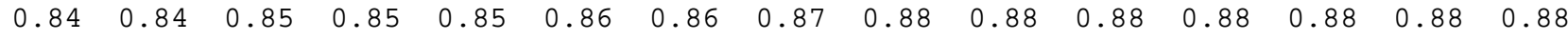


Table Ca-5. Projected fuel price indices (excluding general inflation), by end-use sector and fuel type.

United States Average

Sector and Fuel
Residential
Electricity
Distillate 0il
Natural Gas
Commercial
Electricity
Distillate Oil
Residual Oil
Natural Gas
Coal
Industrial
Electricity
Distillate 0il
Residual Oil
Natural Gas
Coal
Transportation
Motor Gasoline

\begin{tabular}{|c|c|c|c|c|c|c|c|c|c|c|c|c|c|c|}
\hline & --- & - - - - - &.- & - - - - - & - - - - - & ---- & $-\cdots$ & ---- & --- & ---- & - - - - - & --- & $\cdots$ & \\
\hline 2008 & 2009 & 2010 & 2011 & 2012 & 2013 & 2014 & 2015 & 2016 & 2017 & 2018 & 2019 & 2020 & 2021 & 2022 \\
\hline-- & - - - - & - - - - - & - - - - & - - - - - & - & - - - - - & - - - - - & - - - - - & - - - - & - - - - - & - - - - - & - - - - - & - - - - & - - - - - \\
\hline 1.00 & $\odot .99$ & $\odot .98$ & $\odot .97$ & $\odot .96$ & $\odot .95$ & $\odot .95$ & $\odot .94$ & $\odot .95$ & $\odot .95$ & $\odot .96$ & $\odot .96$ & $\odot .96$ & $\odot .96$ & $\odot .96$ \\
\hline 0.98 & $\odot .93$ & $\odot .87$ & $\odot .82$ & $\odot .78$ & $\odot .74$ & $\odot .72$ & $\odot .73$ & $\odot .73$ & 0.73 & $\odot .74$ & $\odot .75$ & $\odot .76$ & $\odot .76$ & 0.77 \\
\hline 1.04 & 1.02 & 1.00 & $\odot .99$ & $\odot .98$ & 0.97 & 0.97 & 0.97 & 0.97 & 0.98 & ๑. 98 & 0.98 & 0.98 & 0.98 & 0.98 \\
\hline 0.97 & $\odot .94$ & ๑. 91 & ๑.88 & 0.86 & 0.85 & 0.85 & $\odot .85$ & 0.85 & 0.87 & 0.87 & ๑.86 & 0.87 & 0.87 & ๑.88 \\
\hline 0.99 & ๑. 98 & 0.97 & 0.94 & 0.93 & $\odot .92$ & 0.92 & $\odot .92$ & 0.92 & 0.93 & 0.94 & $\odot .94$ & $\odot .94$ & 0.94 & $\odot .94$ \\
\hline . 97 & $\odot .9 \odot$ & $\odot .85$ & $\odot .8 \odot$ & $\odot .76$ & $\odot .72$ & $\odot .71$ & $\odot .71$ & $\odot .72$ & $\odot .72$ & $\odot .73$ & $\odot .74$ & $\odot .75$ & $\odot .75$ & 0.77 \\
\hline ๑. 93 & $\odot .92$ & 0.90 & ๑. 86 & 0.83 & 0.74 & 0.74 & $\odot .77$ & 0.77 & 0.78 & ๑. 80 & 0.83 & 0.84 & ๑.86 & 0.88 \\
\hline . 97 & 0.93 & ๑.89 & 0.86 & 0.83 & 0.81 & 0.81 & 0.81 & 0.81 & 0.82 & ๑. 82 & 0.82 & 0.82 & 0.82 & $\odot .83$ \\
\hline 1.01 & 1.00 & 1.01 & 1.01 & 1.00 & $\odot .99$ & $\odot .98$ & $\odot .98$ & $\odot .98$ & $\odot .97$ & $\odot .96$ & 0.96 & $\odot .96$ & $\odot .97$ & 0.97 \\
\hline 1.01 & 1.00 & 0.97 & 0.94 & 0.91 & $\odot .89$ & $\odot .89$ & ๑. 88 & 0.89 & $0.9 \odot$ & 0.91 & 0.91 & 0.91 & 0.92 & 0.92 \\
\hline . 95 & ๑. 89 & ๑.85 & ๑. 82 & 0.78 & 0.76 & 0.74 & 0.74 & 0.75 & 0.76 & 0.76 & 0.78 & 0.78 & $\odot .79$ & 0.80 \\
\hline 1.02 & $\odot .98$ & $\odot .94$ & $\odot .9 \odot$ & $\odot .86$ & $\odot .82$ & $\odot .80$ & $\odot .81$ & $\odot .81$ & $\odot .82$ & 0.83 & $\odot .85$ & $\odot .87$ & ๑.88 & $\odot .9 \odot$ \\
\hline$\odot .99$ & 0.93 & ๑. 88 & ๑.83 & ๑. 80 & 0.78 & 0.77 & 0.77 & 0.78 & 0.80 & $\odot .80$ & 0.79 & 0.80 & ๑. . 80 & 0.81 \\
\hline 1.01 & 1.00 & 1.01 & 1.01 & 1.00 & 0.99 & 0.99 & 0.99 & 0.98 & 0.98 & 0.98 & 0.98 & 0.98 & 0.98 & 0.99 \\
\hline .95 & $\odot .9 \odot$ & ๑. 86 & $\odot .83$ & 0.81 & $\odot .79$ & 0.77 & $\odot .76$ & 0.77 & 0.77 & 0.78 & $\odot .79$ & $\odot .79$ & 0.80 & 0.81 \\
\hline
\end{tabular}


Table Ca-5, continued. Projected fuel price indices (excluding general inflation), by end-use sector and fuel type.

United States Average

Projected April 1 Fuel Price Indices (April 1, $2007=1.00$ )

\begin{tabular}{|c|c|c|c|c|c|c|c|c|c|c|c|c|c|c|c|}
\hline 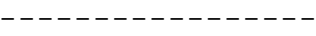 & $\cdots$ & -- & $\cdots-$ & $-\ldots$ & & & & 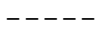 & $\cdots$ & -- & $\cdots$ & 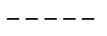 & -- & ---- & - \\
\hline Sector and Fuel & 2023 & 2024 & 2025 & 2026 & 2027 & 2028 & 2029 & 2030 & 2031 & 2032 & 2033 & 2034 & 2035 & 2036 & 2037 \\
\hline - - - - - - - - - - - - - & $-\cdots$ & $-\cdots$ & $-\cdots-$ & $-\cdots$ & --- & --- & $-\cdots-$ & $-\cdots$ & --- & --- & --- & 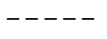 & --- & --- & --- \\
\hline Residential & & & & & & & & & & & & & & & \\
\hline Electricity & 0.96 & 0.96 & 0.97 & 0.97 & 0.97 & 0.97 & $\odot .97$ & 0.97 & 0.97 & 0.98 & 0.98 & 0.98 & 0.98 & 0.98 & 0.99 \\
\hline Distillate Oil & 0.77 & 0.78 & 0.78 & 0.79 & 0.79 & 0.80 & 0.81 & 0.82 & 0.82 & 0.82 & 0.82 & 0.82 & 0.82 & 0.82 & 0.82 \\
\hline LPG & 0.99 & 0.99 & 0.99 & 1.00 & 1.00 & 1.00 & 1.01 & 1.01 & 1.01 & 1.01 & 1.01 & 1.01 & 1.01 & 1.01 & 1.01 \\
\hline Natural Gas & $\odot .89$ & 0.90 & 0.90 & $\odot .91$ & 0.92 & 0.93 & 0.94 & 0.94 & 0.95 & 0.95 & 0.96 & 0.97 & 0.98 & 0.98 & 0.99 \\
\hline Commercial & & & & & & & & & & & & & & & \\
\hline Electricity & 0.95 & 0.95 & 0.95 & 0.95 & 0.95 & 0.95 & 0.95 & 0.95 & 0.96 & 0.96 & 0.96 & 0.96 & 0.96 & 0.96 & 0.97 \\
\hline Distillate Oil & 0.76 & 0.77 & 0.78 & 0.78 & 0.79 & 0.81 & 0.81 & 0.82 & 0.82 & 0.82 & 0.83 & 0.83 & 0.83 & 0.83 & 0.83 \\
\hline Residual Oil & 0.87 & 0.88 & $\odot .86$ & 0.87 & $\odot .88$ & 0.89 & 0.87 & 0.87 & 0.87 & 0.88 & 0.88 & 0.88 & $\odot .88$ & 0.88 & 0.88 \\
\hline Natural Gas & $\odot .84$ & 0.85 & 0.85 & 0.85 & $\odot .86$ & 0.87 & 0.88 & 0.88 & 0.89 & 0.90 & 0.90 & 0.91 & 0.92 & 0.93 & 0.94 \\
\hline Coal & 0.98 & $\odot .98$ & 0.99 & $\odot .99$ & $1 . \odot \odot$ & 1.00 & 1.01 & 1.02 & 1.02 & 1.03 & 1.03 & 1.04 & 1.04 & 1.04 & 1.05 \\
\hline Industrial & & & & & & & & & & & & & & & \\
\hline Electricity & 0.92 & 0.93 & 0.93 & 0.93 & 0.93 & 0.93 & 0.93 & 0.94 & 0.94 & 0.94 & 0.94 & 0.94 & 0.95 & 0.95 & 0.95 \\
\hline Distillate Oil & 0.80 & 0.80 & 0.82 & 0.82 & 0.83 & 0.85 & 0.85 & 0.86 & 0.86 & 0.86 & 0.86 & 0.87 & 0.87 & 0.87 & 0.87 \\
\hline Residual 0il & 0.89 & 0.90 & 0.90 & 0.90 & 0.91 & 0.92 & 0.92 & 0.92 & 0.93 & 0.93 & 0.93 & 0.93 & 0.93 & 0.93 & 0.94 \\
\hline Natural Gas & 0.83 & 0.84 & 0.84 & 0.85 & 0.86 & 0.87 & 0.88 & 0.89 & 0.90 & 0.91 & 0.92 & 0.93 & 0.94 & 0.95 & 0.96 \\
\hline Coal & 0.99 & 1.00 & 1.00 & 1.00 & 1.01 & 1.01 & 1.02 & 1.02 & 1.03 & 1.03 & 1.04 & 1.04 & 1.04 & 1.05 & 1.05 \\
\hline Transportation & & & & & & & & & & & & & & & \\
\hline Motor Gasoline & 0.81 & 0.81 & 0.82 & 0.82 & 0.82 & 0.83 & 0.83 & 0.84 & 0.85 & 0.85 & 0.85 & 0.85 & 0.85 & 0.85 & 0.85 \\
\hline
\end{tabular}


Table Cb-1. Projected average fuel price escalation rates, excluding general inflation, by end-use sector and fuel type.

Census Region 1 (Connecticut, Maine, Massachusetts, New Hampshire, New Jersey, New York, Pennsylvania, Rhode Island, Vermont)

\begin{tabular}{|c|c|c|c|c|c|c|}
\hline & & Percen & change & ipounde & inually & \\
\hline - - - - - - - - - - - & $-\ldots$ & ---- & ---- & ---- & ---- & $-\ldots$ \\
\hline & 2007 & 2012 & 2017 & 2022 & 2027 & 2032 \\
\hline Sector and Fuel & to & to & to & to & to & to \\
\hline & 2012 & 2017 & $2 \odot 22$ & 2027 & 2032 & 2037 \\
\hline - - - - - - - - - - - - - & ---- & --- & ---- & $-\ldots$ & $-\ldots$ & --- \\
\hline Residential & & & & & & \\
\hline Electricity & -0.8 & 0.2 & 0.2 & 0.2 & 0.3 & 0.2 \\
\hline Distillate oil & -4.7 & -1.2 & 1.0 & 0.5 & 0.6 & 0.1 \\
\hline LPG & -0.6 & -0.1 & $\odot .0$ & $\odot .2$ & $\odot .2$ & 0.1 \\
\hline Natural Gas & -2.7 & -0.0 & 0.3 & 0.7 & $\odot .8$ & 0.6 \\
\hline Commercial & & & & & & \\
\hline Electricity & -2.9 & 0.3 & 0.2 & 0.1 & 0.1 & 0.1 \\
\hline Distillate oil & -5.2 & -1.0 & 1.3 & 0.6 & 0.8 & 0.1 \\
\hline Residual oil & -3.8 & -1.3 & 2.5 & -0.1 & -0.2 & 0.2 \\
\hline Natural Gas & -3.7 & -0.3 & 0.0 & 0.6 & 0.8 & 0.8 \\
\hline Coal & -1.2 & -1.8 & 0.1 & $\odot .8$ & $\odot .8$ & 0.4 \\
\hline Industrial & & & & & & \\
\hline Electricity & -4.8 & -0.5 & 0.4 & $\odot .3$ & $\odot .2$ & 0.2 \\
\hline Distillate oil & -4.7 & -0.7 & 1.3 & 0.6 & 0.9 & 0.1 \\
\hline Residual 0il & -2.8 & -1.1 & 2.2 & -0.1 & -0.2 & 0.2 \\
\hline Natural Gas & -4.5 & -0.4 & 0.1 & 0.9 & 1.1 & 1.1 \\
\hline Coal & 0.1 & -1.3 & -0.2 & 0.6 & 0.6 & 0.4 \\
\hline Transportation & & & & & & \\
\hline Motor Gasoline & -4.4 & -0.8 & 0.9 & 0.3 & $\odot .6$ & 0.1 \\
\hline - - - - - - - - - - - - - - & ---- & ---- & ---- & $-\cdots$ & 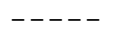 & ---- \\
\hline
\end{tabular}


Table Cb-2. Projected average fuel price escalation rates, excluding general inflation, by end-use sector and fuel type.

\begin{tabular}{|c|c|c|c|c|c|c|}
\hline \multirow{2}{*}{ 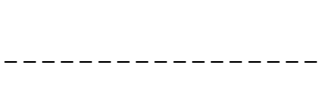 } & \multicolumn{5}{|c|}{ Percentage change compounded annually } & \multirow[b]{2}{*}{----} \\
\hline & - - - - & $-\ldots$ & -- & --- & $-\ldots$ & \\
\hline \multirow[b]{2}{*}{ Sector and Fuel } & $20 \odot 7$ & 2012 & 2017 & 2022 & 2027 & 2032 \\
\hline & $\begin{array}{c}\text { to } \\
2012\end{array}$ & $\begin{array}{c}\text { to } \\
2017\end{array}$ & $\begin{array}{c}\text { to } \\
2 \odot 22\end{array}$ & $\begin{array}{c}\text { to } \\
2027\end{array}$ & $\begin{array}{c}\text { to } \\
2032\end{array}$ & $\begin{array}{c}\text { to } \\
2037\end{array}$ \\
\hline \multirow{2}{*}{\multicolumn{7}{|c|}{ Residential }} \\
\hline & & & & & & \\
\hline Electricity & 0.3 & -0.1 & 0.2 & -0.0 & 0.1 & 0.0 \\
\hline Distillate oil & -5.7 & -1.6 & 1.2 & 0.5 & 0.6 & 0.1 \\
\hline LPG & -0.2 & $-\odot . \odot$ & 0.1 & 0.3 & 0.3 & 0.1 \\
\hline Natural Gas & -3.1 & 0.1 & 0.4 & 0.9 & 0.9 & 0.8 \\
\hline \multicolumn{7}{|l|}{ Commercial } \\
\hline Electricity & -0.0 & -0.1 & $\odot .6$ & 0.2 & 0.1 & 0.2 \\
\hline Distillate $0 i l$ & -5.8 & -1.2 & 1.3 & 0.6 & 0.8 & 0.1 \\
\hline Residual Oil & -1.6 & -0.7 & 1.2 & 0.5 & 0.5 & 0.1 \\
\hline Natural Gas & -3.6 & -0.2 & 0.2 & 0.7 & 0.8 & 0.9 \\
\hline Coal & 1.2 & -0.5 & -0.3 & 0.5 & 0.4 & 0.4 \\
\hline \multicolumn{7}{|l|}{ Industrial } \\
\hline Electricity & -1.2 & -0.3 & $\odot .7$ & $\odot .2$ & 0.2 & 0.2 \\
\hline Distillate $0 i l$ & -4.7 & -0.7 & 1.2 & 0.5 & $\odot .8$ & 0.1 \\
\hline Residual 0il & -1.7 & $-\odot .7$ & 1.2 & 0.5 & 0.5 & 0.2 \\
\hline Natural Gas & -3.5 & -0.2 & 0.2 & 1.0 & 1.1 & 1.1 \\
\hline Coal & 0.8 & -0.4 & $\odot .3$ & 0.5 & $\odot .5$ & 0.5 \\
\hline \multicolumn{7}{|l|}{ Transportation } \\
\hline Motor Gasoline & -4.3 & -0.9 & 0.9 & 0.3 & 0.6 & 0.1 \\
\hline - - - - - - - - - - - - - & ---- & ---- & --- & ---- & ---- & --- \\
\hline
\end{tabular}


Table Cb-3. Projected average fuel price escalation rates, excluding general inflation, by end-use sector and fuel type.

Census Region 3 (Alabama, Arkansas, Delaware, District of Columbia, Florida, Georgia, Kentucky, Louisiana, Maryland, Mississippi, North Carolina, Oklahoma, South Carolina, Tennessee, Texas, Virginia, West Virginia)

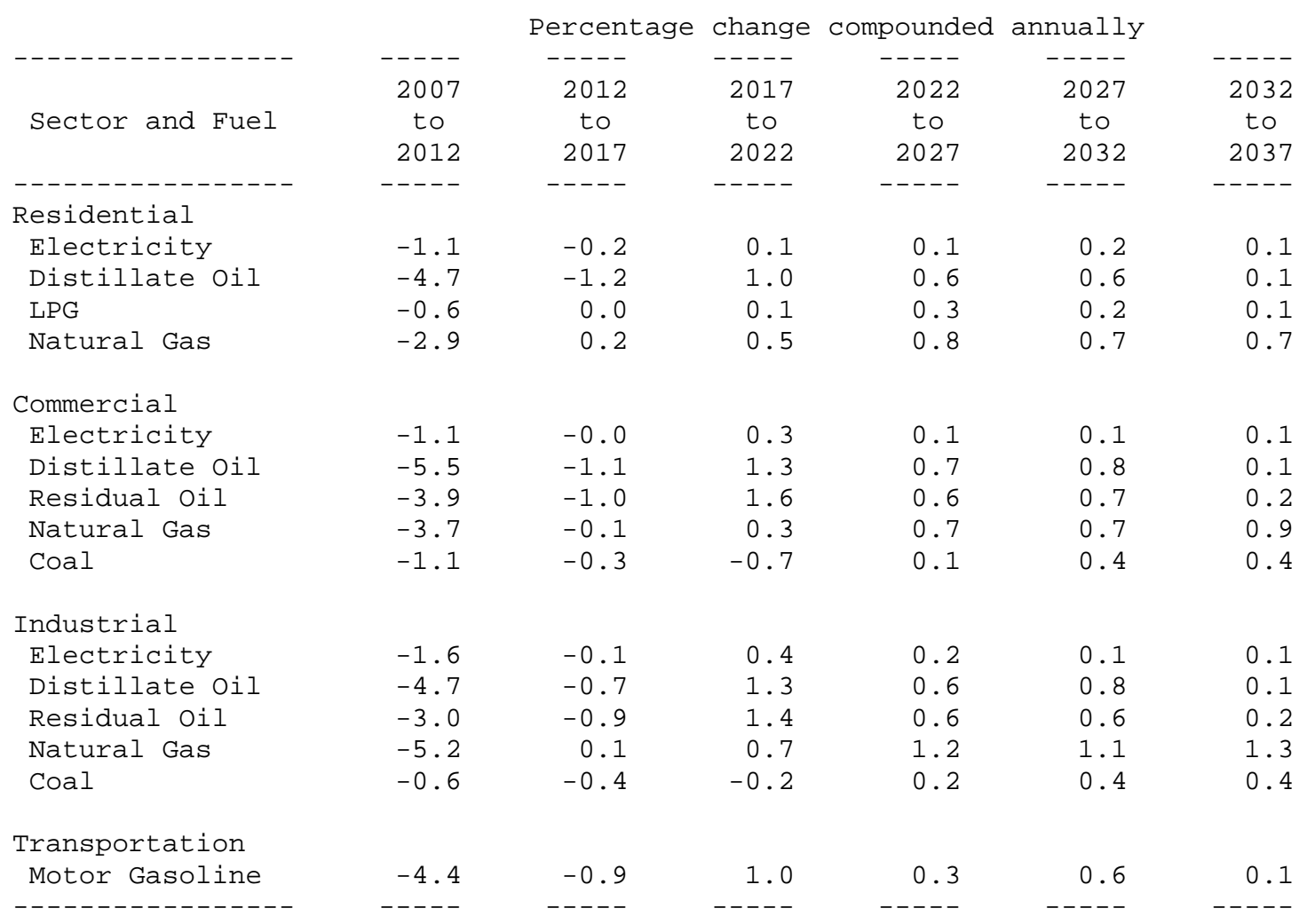


Table Cb-4. Projected average fuel price escalation rates, excluding general inflation, by end-use sector and fuel type.

Census Region 4 (Alaska, Arizona, California, Colorado, Hawaii, Idaho, Montana, Nevada, New Mexico, Oregon, Utah, Washington, Wyoming)

\begin{tabular}{|c|c|c|c|c|c|c|}
\hline \multirow{3}{*}{ - - - - - - - - - - - - } & & \multicolumn{5}{|c|}{ annually } \\
\hline & $-\ldots$ & ---- & $-\ldots$ & $-\ldots$ & ---- & $-\ldots$ \\
\hline & $20 \odot 7$ & 2012 & 2017 & 2022 & 2027 & 2032 \\
\hline \multirow[t]{2}{*}{ Sector and Fuel } & to & to & to & to & to & to \\
\hline & 2012 & 2017 & 2022 & 2027 & 2032 & 2037 \\
\hline - - - - - - - - - - - & $-\ldots$ & $-\ldots$ & $-\ldots$ & $-\ldots$ & $-\ldots$ & $-\ldots$ \\
\hline \multicolumn{7}{|l|}{ Residential } \\
\hline Electricity & -1.4 & 0.2 & 0.1 & 0.3 & 0.4 & 0.4 \\
\hline Distillate $0 i l$ & -5.3 & -1.3 & 1.6 & 1.0 & 0.7 & 0.1 \\
\hline LPG & -0.4 & -0.1 & -0.1 & 0.4 & 0.3 & 0.1 \\
\hline Natural Gas & -2.5 & -0.1 & -0.2 & 1.0 & 1.0 & 0.8 \\
\hline \multicolumn{7}{|l|}{ Commercial } \\
\hline Electricity & -1.9 & 0.1 & -0.1 & 0.2 & 0.3 & 0.3 \\
\hline Distillate $0 i l$ & -5.5 & -1.0 & 1.3 & 0.8 & 0.8 & 0.1 \\
\hline Residual oil & -5.8 & -1.7 & 2.1 & 0.9 & 0.8 & 0.2 \\
\hline Natural Gas & -3.0 & -0.4 & -0.5 & 0.9 & 1.5 & 0.9 \\
\hline Coal & 0.4 & 0.3 & 1.2 & 0.8 & 0.6 & 0.4 \\
\hline \multicolumn{7}{|l|}{ Industrial } \\
\hline Electricity & -1.6 & 0.1 & $\odot . \odot$ & 0.3 & $\odot .5$ & 0.4 \\
\hline Distillate oil & -5.0 & -0.6 & 0.9 & 0.7 & 0.8 & 0.1 \\
\hline Residual $0 i 1$ & -3.6 & -1.0 & 1.3 & 1.1 & 0.4 & 0.2 \\
\hline Natural Gas & -3.4 & -0.4 & -0.4 & 1.3 & 1.5 & 1.2 \\
\hline Coal & -0.1 & 0.1 & 1.5 & 0.9 & 0.7 & 0.4 \\
\hline \multicolumn{7}{|l|}{ Transportation } \\
\hline Motor Gasoline & -3.5 & -0.8 & 0.9 & 0.3 & 0.6 & 0.1 \\
\hline - & $-\ldots$ & $-\ldots$ & $-\ldots$ & $-\ldots$ & $-\ldots$ & $\ldots$ \\
\hline
\end{tabular}


Table Cb-5. Projected average fuel price escalation rates, excluding general inflation, by end-use sector and fuel type.

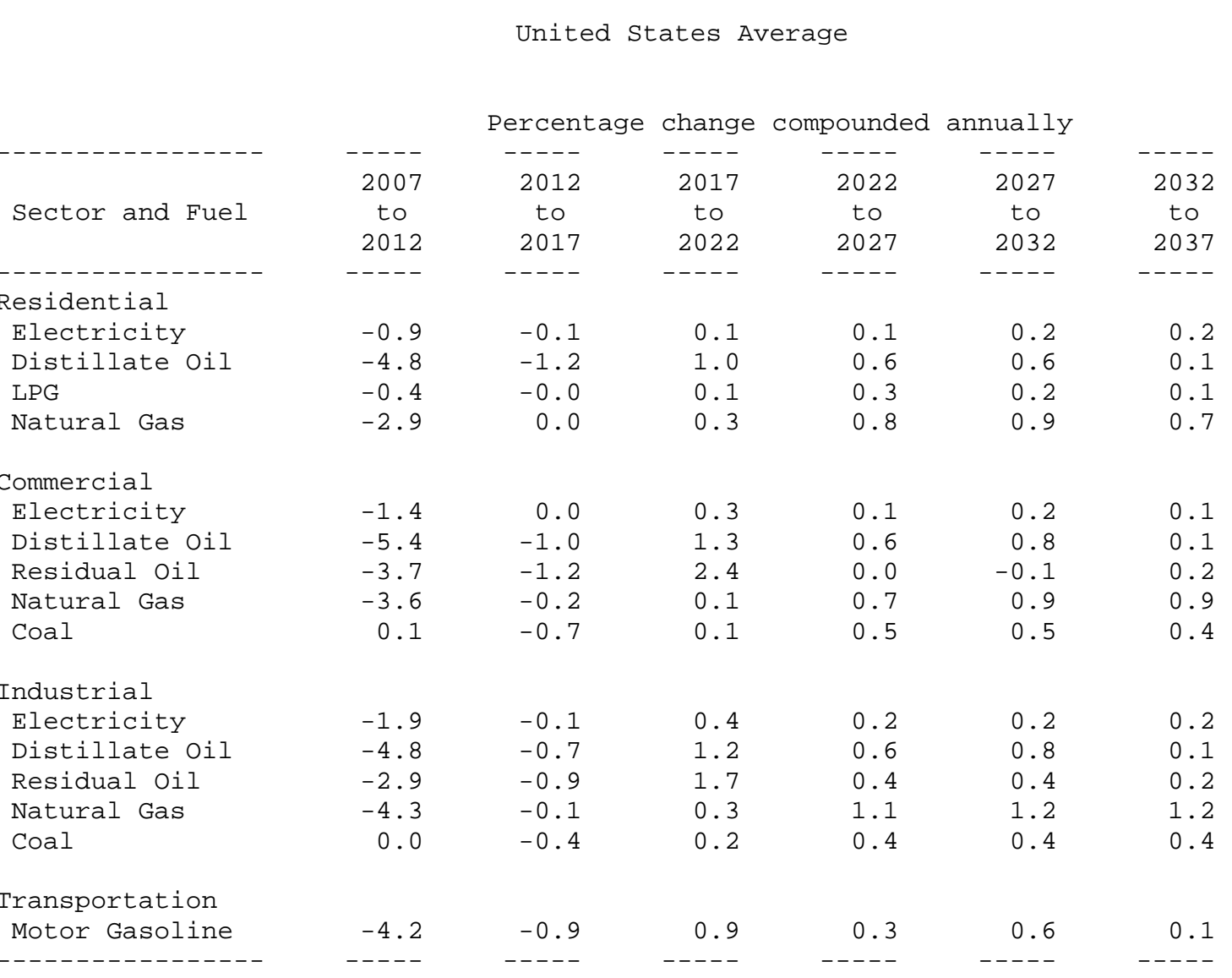




\section{PART II: \\ ENERGY PRICE INDICES FOR PRIVATE SECTOR LCC ANALYSIS}

This section presents tables of projected nominal (i.e., including inflation) fuel price indices for four fuels in the residential sector and five fuels in the commercial sector for each of the years from 2007 through 2037. These price indices are based on the DOE energy price projections, reported in Part I, Section B of this document, used to calculate the FEMP and OMB UPV* factors for energy costs. Tables S-1 to S-5 are provided as an update to similar tables originally published in Comprehensive Guide for Least-Cost Energy Decisions (NBS SP 709).

As a convenience for the user, the indices include the effect of four alternative, hypothetical rates of general price inflation: $2 \%, 3 \%, 4 \%$, and $5 \%$. Selection of these rates is in no way intended to suggest what actual rates might be. Use of the indices produce price estimates in current dollars, inclusive of general price inflation. Current-dollar prices are needed when discounting is performed with discount rates that include general price inflation (i.e., nominal or market discount rates).

The calculated indices with inflation rates of $2 \%, 3 \%, 4 \%$, and $5 \%$ allow the analyst to perform evaluations based on the assumption of a positive rate of general price inflation that changes the purchasing power of the dollar. Performing evaluations in current dollars is sometimes preferred for private investment decisions, primarily because it facilitates the treatment of taxes.

The indices in Tables S-1 through S-5 are derived from the indices reported in Tables Ca-1 through Ca- 5 by means of the following equation:

$$
I_{S}=I_{C} \times(1+g)^{N},
$$

where $\mathrm{I}_{\mathrm{S}}=$ index found in Tables S-1 through S-5;

$\mathrm{I}_{\mathrm{C}}=$ index found in Tables Ca-1 through Ca-5;

$\mathrm{g}=$ annual rate of general price inflation in decimal form; and

$\mathrm{N}=$ number of years, in this case equal to the year of the index minus 2007.

\section{Example of How to Use the Indices:}

Suppose you wish to estimate the annual cost of natural gas for a house in Maryland in year 2011, given the annual cost in 2007 (April 1) prices, and you expect an annual inflation rate of $3 \%$ per year. From table S-3, find the column with residential natural gas indices at an inflation rate of $3 \%$; then locate the index for the year 2011 . This index is 0.99 . Multiply the annual cost in 2007 prices by the index to find the estimated annual cost in year 2011 prices.

If this annual cost in year-2011 prices is to be discounted to present value, you must use a nominal discount rate that includes the same assumption with regard to general price inflation ( $3 \%$ in this example). To obtain a present-value cost over the entire study period, the presentvalue calculation must be repeated for each year that there are natural gas costs, and the results summed. (UPV* factors are not given for private sector use because of the large number of 
tables required to cover potential discount rates that might be used by the analyst.) The BLCC computer program can perform LCC analyses using any inflation rate and discount rate, in constant or in current (market) dollars. The DISCOUNT program, included with BLCC, can compute UPV* factors based on DOE energy price escalation rates using any inflation rate and discount rate. (See page iv for more information on these programs.) Of course, the private sector analyst may use the UPV* factors reported in Part I, provided the analysis is performed in constant dollars and the desired discount rate corresponds to the DOE or OMB discount rates used in Part I.

For further explanation of the use of these indices, see NBS Special Publication 709, appendix B, Part I.

The data in the tables that follow are reported for the four Census regions and the U.S. average. Figure B-1 on page 13 presents a map showing the states corresponding to the four Census regions. The Census regions do not include American Samoa, Canal Zone, Guam, Puerto Rico, Trust Territory of the Pacific Islands, or the Virgin Islands. Analysts of federal projects in these areas should use data which are "reasonable under the circumstances," and may refer to the tables with U.S. average data for guidance. 
Table S-1. Projected fuel price indices with assumed general price inflation rates of $2 \%, 3 \%, 4 \%$, and $5 \%$, by end-use sector and fuel type.

Census Region 1 (Connecticut, Maine, Massachusetts, New Hampshire, New Jersey, New York, Pennsylvania, Rhode Island, Vermont)

Projected April 1 Fuel Price Indices (April 1, $2007=1.00$ )

\begin{tabular}{|c|c|c|c|c|}
\hline & \multicolumn{4}{|c|}{ Electricity } \\
\hline & \multicolumn{4}{|c|}{ Inflation Rate } \\
\hline Year & $2 \%$ & $3 \%$ & $4 \%$ & $5 \%$ \\
\hline $20 \odot 8$ & $1.0 \odot$ & 1.01 & 1.02 & 1.03 \\
\hline $20 \odot 9$ & 1.02 & 1.04 & 1.06 & 1.08 \\
\hline 2010 & 1.02 & 1.06 & 1.09 & 1.12 \\
\hline 2011 & 1.03 & 1.08 & 1.12 & 1.16 \\
\hline 2012 & 1.06 & 1.11 & 1.17 & 1.22 \\
\hline 2013 & 1.09 & 1.15 & 1.22 & 1.29 \\
\hline 2014 & 1.11 & 1.18 & 1.27 & 1.35 \\
\hline 2015 & 1.13 & 1.22 & 1.32 & 1.43 \\
\hline 2016 & 1.15 & 1.26 & 1.37 & 1.50 \\
\hline 2017 & 1.18 & 1.30 & 1.43 & 1.58 \\
\hline 2018 & 1.22 & 1.35 & 1.50 & 1.67 \\
\hline 2019 & 1.24 & 1.39 & 1.57 & 1.76 \\
\hline 2020 & 1.26 & 1.44 & 1.63 & 1.84 \\
\hline 2021 & 1.29 & 1.48 & 1.70 & 1.94 \\
\hline 2022 & 1.32 & 1.53 & 1.77 & 2.04 \\
\hline 2023 & 1.35 & 1.58 & 1.84 & 2.15 \\
\hline 2024 & 1.39 & 1.64 & 1.93 & 2.27 \\
\hline 2025 & 1.42 & 1.69 & 2.01 & 2.39 \\
\hline 2026 & 1.45 & 1.74 & 2.09 & 2.51 \\
\hline 2027 & 1.48 & 1.79 & 2.18 & 2.63 \\
\hline 2028 & 1.51 & 1.86 & 2.27 & 2.78 \\
\hline 2029 & 1.55 & 1.92 & 2.37 & 2.93 \\
\hline 2030 & 1.58 & 1.98 & 2.47 & 3.08 \\
\hline 2031 & 1.61 & 2.04 & 2.57 & 3.23 \\
\hline 2032 & 1.65 & 2.11 & 2.68 & 3.41 \\
\hline 2033 & 1.69 & 2.17 & 2.79 & 3.58 \\
\hline 2034 & 1.72 & 2.24 & 2.91 & 3.77 \\
\hline 2035 & 1.76 & 2.32 & 3.04 & 3.97 \\
\hline 2036 & 1.80 & 2.39 & 3.17 & 4.18 \\
\hline 2037 & 1.84 & 2.47 & 3.30 & 4.40 \\
\hline
\end{tabular}

\begin{tabular}{rrrr}
\multicolumn{4}{c}{ Distillate 0 il } \\
\multicolumn{4}{c}{ Inflation Rate } \\
$2 \%$ & $3 \% \%$ & $4 \%$ & $5 \%$ \\
1.01 & 1.02 & 1.03 & 1.03 \\
0.97 & 0.99 & 1.01 & 1.03 \\
0.93 & 0.95 & 0.98 & 1.01 \\
0.89 & 0.93 & 0.96 & 1.00 \\
0.87 & 0.91 & 0.96 & 1.09 \\
0.84 & 0.89 & 0.94 & 0.99 \\
0.84 & $0.9 \odot$ & 0.96 & 1.03 \\
0.86 & 0.93 & 1.00 & 1.08 \\
0.88 & 0.96 & 1.05 & 1.14 \\
0.90 & 0.99 & 1.09 & 1.20 \\
0.93 & 1.03 & 1.15 & 1.27 \\
0.96 & 1.08 & 1.21 & 1.35 \\
0.99 & 1.12 & 1.27 & 1.44 \\
1.01 & 1.16 & 1.33 & 1.52 \\
1.04 & 1.21 & 1.40 & 1.61 \\
1.06 & 1.24 & 1.45 & 1.69 \\
1.09 & 1.29 & 1.52 & 1.79 \\
1.12 & 1.34 & 1.59 & 1.89 \\
1.15 & 1.38 & 1.66 & 1.99 \\
1.19 & 1.44 & 1.75 & 2.12 \\
1.22 & 1.50 & 1.83 & 2.24 \\
1.26 & 1.56 & 1.93 & 2.38 \\
1.29 & 1.61 & 2.01 & 2.51 \\
1.32 & 1.66 & 2.10 & 2.64 \\
1.35 & 1.72 & 2.19 & 2.78 \\
1.37 & 1.77 & 2.28 & 2.92 \\
1.40 & 1.83 & 2.37 & 3.07 \\
1.43 & 1.88 & 2.47 & 3.22 \\
1.46 & 1.94 & 2.57 & 3.39 \\
1.49 & $2.0 \odot$ & 2.67 & 3.56
\end{tabular}

\begin{tabular}{rrrr}
\multicolumn{4}{c}{ LPG } \\
\multicolumn{4}{c}{ Inflation Rate } \\
$2 \%$ & $3 \%$ & $4 \%$ & $5 \%$ \\
1.05 & 1.06 & 1.07 & 1.08 \\
1.05 & 1.07 & 1.09 & 1.11 \\
1.05 & 1.08 & 1.12 & 1.15 \\
1.06 & 1.10 & 1.14 & 1.19 \\
1.07 & 1.12 & 1.18 & 1.24 \\
1.08 & 1.15 & 1.21 & 1.29 \\
1.10 & 1.18 & 1.26 & 1.35 \\
1.12 & 1.21 & 1.31 & 1.41 \\
1.14 & 1.25 & 1.36 & 1.48 \\
1.17 & 1.29 & 1.43 & 1.57 \\
1.20 & 1.33 & 1.48 & 1.65 \\
1.22 & 1.37 & 1.54 & 1.73 \\
1.25 & 1.41 & 1.60 & 1.82 \\
1.27 & 1.45 & 1.67 & 1.90 \\
1.30 & 1.50 & 1.74 & 2.01 \\
1.33 & 1.55 & 1.81 & 2.12 \\
1.36 & 1.61 & 1.89 & 2.23 \\
1.39 & 1.66 & 1.97 & 2.34 \\
1.42 & 1.71 & 2.05 & 2.46 \\
1.45 & 1.76 & 2.14 & 2.59 \\
1.49 & 1.82 & 2.23 & 2.73 \\
1.52 & 1.88 & 2.33 & 2.88 \\
1.55 & 1.94 & 2.43 & 3.03 \\
1.59 & 2.00 & 2.53 & 3.18 \\
1.62 & 2.06 & 2.63 & 3.34 \\
1.65 & 2.13 & 2.74 & 3.51 \\
1.69 & 2.19 & 2.85 & 3.69 \\
1.72 & 2.26 & 2.96 & 3.87 \\
1.76 & 2.33 & 3.08 & 4.07 \\
1.79 & 2.40 & 3.21 & 4.27
\end{tabular}

Natural Gas

\begin{tabular}{rrrr}
\multicolumn{4}{c}{ Inflation Rate } \\
$2 \%$ & $3 \%$ & $4 \%$ & $5 \%$ \\
0.99 & 1.00 & 1.01 & 1.02 \\
0.98 & 1.00 & 1.02 & 1.04 \\
0.97 & 1.00 & 1.03 & 1.06 \\
0.96 & 1.00 & 1.04 & 1.08 \\
0.96 & 1.01 & 1.06 & 1.11 \\
0.96 & 1.02 & 1.08 & 1.15 \\
0.98 & 1.05 & 1.12 & 1.20 \\
1.00 & 1.08 & 1.17 & 1.26 \\
1.02 & 1.11 & 1.21 & 1.32 \\
1.06 & 1.17 & 1.29 & 1.42 \\
1.08 & 1.21 & 1.34 & 1.49 \\
1.10 & 1.24 & 1.39 & 1.56 \\
1.13 & 1.29 & 1.46 & 1.65 \\
1.15 & 1.32 & 1.51 & 1.73 \\
1.19 & 1.37 & 1.59 & 1.83 \\
1.22 & 1.43 & 1.67 & 1.95 \\
1.26 & 1.49 & 1.75 & 2.06 \\
1.29 & 1.54 & 1.83 & 2.17 \\
1.32 & 1.59 & 1.91 & 2.29 \\
1.36 & 1.65 & 2.00 & 2.42 \\
1.40 & 1.72 & 2.10 & 2.57 \\
1.44 & 1.79 & 2.21 & 2.73 \\
1.48 & 1.85 & 2.31 & 2.88 \\
1.52 & 1.92 & 2.42 & 3.04 \\
1.56 & 1.99 & 2.53 & 3.21 \\
1.60 & 2.06 & 2.65 & 3.39 \\
1.64 & 2.13 & 2.77 & 3.58 \\
1.68 & 2.21 & 2.90 & 3.79 \\
1.73 & 2.29 & 3.03 & 4.00 \\
1.77 & 2.37 & 3.17 & 4.23
\end{tabular}


Table S-1, continued. Projected fuel price indices with assumed general price inflation rates of $2 \%, 3 \%, 4 \%$, and $5 \%$, by end-use sector and fuel type.

Census Region 1 (Connecticut, Maine, Massachusetts, New Hampshire,

New Jersey, New York, Pennsylvania, Rhode Island, Vermont)

Projected April 1 Fuel Price Indices (April 1, $2007=1.00$ )

\begin{tabular}{rrrrr}
\multicolumn{4}{c}{ Electricity } \\
Year & $2 \%$ & $3 \%$ & $4 \%$ & $5 \%$ \\
2008 & 0.99 & 1.00 & 1.01 & 1.02 \\
2009 & 0.98 & 1.00 & 1.02 & 1.04 \\
2010 & 0.96 & 0.99 & 1.02 & 1.05 \\
2011 & 0.94 & 0.98 & 1.01 & 1.05 \\
2012 & 0.95 & 1.00 & 1.05 & 1.10 \\
2013 & 0.97 & 1.03 & 1.10 & 1.16 \\
2014 & 0.99 & 1.06 & 1.13 & 1.21 \\
2015 & 1.01 & 1.10 & 1.18 & 1.28 \\
2016 & 1.04 & 1.13 & 1.24 & 1.35 \\
2017 & 1.07 & 1.18 & 1.29 & 1.42 \\
2018 & 1.10 & 1.23 & 1.36 & 1.51 \\
2019 & 1.12 & 1.26 & 1.42 & 1.59 \\
2020 & 1.15 & 1.30 & 1.48 & 1.67 \\
2021 & 1.17 & 1.34 & 1.54 & 1.76 \\
2022 & 1.19 & 1.38 & 1.59 & 1.84 \\
2023 & 1.22 & 1.43 & 1.67 & 1.94 \\
2024 & 1.25 & 1.48 & 1.74 & 2.05 \\
2025 & 1.28 & 1.52 & 1.81 & 2.16 \\
2026 & 1.30 & 1.57 & 1.88 & 2.26 \\
2027 & 1.32 & 1.61 & 1.95 & 2.36 \\
2028 & 1.36 & 1.67 & 2.05 & 2.50 \\
2029 & 1.39 & 1.72 & 2.13 & 2.63 \\
2030 & 1.41 & 1.77 & 2.20 & 2.75 \\
2031 & 1.44 & 1.81 & 2.29 & 2.88 \\
2032 & 1.47 & 1.87 & 2.38 & 3.03 \\
2033 & 1.50 & 1.93 & 2.48 & 3.18 \\
2034 & 1.53 & 1.99 & 2.58 & 3.34 \\
2035 & 1.56 & 2.05 & 2.68 & 3.51 \\
2036 & 1.59 & 2.11 & 2.79 & 3.69 \\
2037 & 1.62 & 2.18 & 2.91 & 3.87
\end{tabular}

Distillate 0il

Inflation Rate

$2 \% 3 \% \quad 4 \% 5 \%$

$0.99 \quad 1.00 \quad 1.01 \quad 1.02$

$0.94 \quad 0.96 \quad 0.98 \quad 1.00$

$\begin{array}{llll}0.91 & 0.93 & 0.96 & 0.99\end{array}$

$0.88 \quad 0.91 \quad 0.95 \quad 0.98$

$0.85 \quad 0.89 \quad 0.93 \quad 0.98$

$\begin{array}{llll}0.82 & 0.87 & 0.93 & 0.98\end{array}$

$0.82 \quad 0.88 \quad 0.94 \quad 1.01$

$0.84 \quad 0.91 \quad 0.98 \quad 1.06$

$0.87 \quad 0.95 \quad 1.03 \quad 1.13$

$\begin{array}{llll}0.89 & 0.98 & 1.08 & 1.19\end{array}$

$\begin{array}{llll}0.91 & 1.02 & 1.13 & 1.26\end{array}$

$\begin{array}{llll}0.95 & 1.07 & 1.20 & 1.35\end{array}$

$0.98 \quad 1.11 \quad 1.26 \quad 1.43$

$\begin{array}{llll}1.01 & 1.16 & 1.32 & 1.51\end{array}$

$\begin{array}{llll}1.05 & 1.21 & 1.40 & 1.62\end{array}$

$\begin{array}{llll}1.06 & 1.24 & 1.45 & 1.69\end{array}$

$\begin{array}{llll}1.09 & 1.29 & 1.52 & 1.78\end{array}$

$\begin{array}{llll}1.13 & 1.34 & 1.60 & 1.90\end{array}$

$\begin{array}{llll}1.13 & 1.34 & 1.60 & 1.90 \\ 1.15 & 1.39 & 1.67 & 2.00\end{array}$

$\begin{array}{llll}1.19 & 1.45 & 1.76 & 2.13\end{array}$

$\begin{array}{llll}1.24 & 1.52 & 1.86 & 2.27\end{array}$

$\begin{array}{llll}1.27 & 1.58 & 1.95 & 2.41\end{array}$

$\begin{array}{llll}1.31 & 1.64 & 2.05 & 2.55\end{array}$

$\begin{array}{llll}1.34 & 1.69 & 2.13 & 2.69\end{array}$

$\begin{array}{llll}1.37 & 1.74 & 2.22 & 2.82\end{array}$

$\begin{array}{llll}1.37 & 1.74 & 2.22 & 2.82 \\ 1.40 & 1.80 & 2.31 & 2.97\end{array}$

$\begin{array}{llll}1.40 & 1.80 & 2.31 & 2.97 \\ 1.43 & 1.86 & 2.41 & 3.12\end{array}$

$\begin{array}{llll}1.46 & 1.91 & 2.51 & 3.28\end{array}$

$\begin{array}{llll}1.49 & 1.97 & 2.61 & 3.45\end{array}$

$\begin{array}{llll}1.52 & 2.03 & 2.72 & 3.62\end{array}$
- - COMMERCIAL -

\begin{tabular}{|c|c|c|c|}
\hline \multicolumn{4}{|c|}{ Inflation Rate } \\
\hline $2 \%$ & $3 \%$ & $4 \%$ & $5 \%$ \\
\hline 0.93 & 0.94 & 0.95 & 0.96 \\
\hline 0.9 & 0.96 & & \\
\hline 0.95 & 0.98 & 1. & 1.04 \\
\hline .92 & 0.95 & & \\
\hline 1 & & & \\
\hline .82 & 0.87 & $\odot$. & 0.97 \\
\hline & 0.90 & & \\
\hline 0.8 & 0.96 & 1. & \\
\hline$\odot .91$ & $\odot .99$ & 1. & \\
\hline 0.94 & 1.0 & 1. & 26 \\
\hline .9 & 1.09 & & \\
\hline 1.04 & 1.17 & 1. & 1.47 \\
\hline 1.08 & 1.23 & 1. & 1.58 \\
\hline .1 & 1.29 & & 1. \\
\hline 1.18 & 1.36 & 1. & 1.8 \\
\hline 1.1 & 1.39 & 1. & 1.89 \\
\hline & & & \\
\hline 1.22 & 1.45 & 1. & 2. \\
\hline 1.25 & 1.51 & 1.8 & 2.17 \\
\hline 1.29 & 1.57 & 1. & 2.31 \\
\hline 1 & 1.6 & & \\
\hline 1.33 & 1.65 & $2 . c$ & 2.51 \\
\hline 1.35 & 1.69 & 2.1 & 2.63 \\
\hline 1.3 & 1.75 & & \\
\hline 1.42 & 1.81 & 2.30 & \\
\hline 1.45 & 1.86 & 2.3 & \\
\hline 1.48 & 1.9 & 2. & \\
\hline & & 2. & \\
\hline 1.54 & & & \\
\hline & & & \\
\hline
\end{tabular}

\begin{tabular}{rrrr}
\multicolumn{4}{c}{ Natural Gas } \\
\multicolumn{4}{c}{ Inflation Rate } \\
$2 \%$ & $3 \%$ & $4 \%$ & $5 \%$ \\
0.98 & 0.99 & 1.00 & 1.01 \\
0.96 & 0.98 & 0.99 & 1.01 \\
0.94 & 0.97 & 1.00 & 1.03 \\
0.92 & 0.96 & 1.00 & 1.04 \\
0.92 & 0.96 & 1.01 & 1.06 \\
0.91 & 0.97 & 1.02 & 1.08 \\
0.92 & 0.99 & 1.06 & 1.13 \\
0.94 & 1.01 & 1.09 & 1.18 \\
0.96 & 1.05 & 1.14 & 1.24 \\
0.99 & 1.10 & 1.21 & 1.33 \\
1.02 & 1.13 & 1.26 & 1.40 \\
1.03 & 1.16 & 1.30 & 1.46 \\
1.06 & 1.20 & 1.36 & 1.54 \\
1.07 & 1.23 & 1.41 & 1.61 \\
1.10 & 1.27 & 1.47 & 1.70 \\
1.14 & 1.33 & 1.55 & 1.81 \\
1.17 & 1.38 & 1.63 & 1.92 \\
1.19 & 1.42 & 1.69 & 2.01 \\
1.22 & 1.47 & 1.76 & 2.12 \\
1.25 & 1.52 & 1.85 & 2.24 \\
1.29 & 1.59 & 1.95 & 2.38 \\
1.33 & 1.65 & 2.04 & 2.52 \\
1.36 & 1.70 & 2.13 & 2.65 \\
1.40 & 1.77 & 2.23 & 2.80 \\
1.44 & 1.83 & 2.33 & 2.96 \\
1.48 & 1.90 & 2.45 & 3.14 \\
1.52 & 1.98 & 2.56 & 3.32 \\
1.56 & 2.05 & 2.69 & 3.52 \\
1.61 & 2.13 & 2.82 & 3.72 \\
1.65 & 2.21 & 2.96 & 3.94
\end{tabular}

Coal

$2 \% 3 \% \quad 4 \% 5 \%$

$\begin{array}{llll}1.02 & 1.03 & 1.04 & 1.05\end{array}$

$\begin{array}{llll}1.03 & 1.05 & 1.07 & 1.09\end{array}$

$\begin{array}{llll}1.04 & 1.07 & 1.10 & 1.13\end{array}$

$\begin{array}{llll}1.05 & 1.09 & 1.13 & 1.17\end{array}$

$\begin{array}{llll}1.04 & 1.09 & 1.14 & 1.20\end{array}$

$\begin{array}{llll}1.03 & 1.09 & 1.16 & 1.23\end{array}$

$\begin{array}{llll}1.03 & 1.011 & 1.18 & 1.26\end{array}$

$\begin{array}{llll}1.04 & 1.12 & 1.21 & 1.31\end{array}$

$\begin{array}{llll}1.04 & 1.14 & 1.24 & 1.35\end{array}$

$\begin{array}{llll}1.05 & 1.16 & 1.27 & 1.40\end{array}$

$\begin{array}{llll}1.05 & 1.17 & 1.31 & 1.45\end{array}$

$\begin{array}{llll}1.07 & 1.20 & 1.35 & 1.52\end{array}$

$\begin{array}{llll}1.10 & 1.25 & 1.42 & 1.61\end{array}$

$\begin{array}{llll}1.14 & 1.30 & 1.49 & 1.70\end{array}$

$\begin{array}{llll}1.16 & 1.35 & 1.56 & 1.80\end{array}$

$1.20 \quad 1.40 \quad 1.64 \quad 1.80$

$\begin{array}{llll}1.20 & 1.40 & 1.64 & 1.91\end{array}$

$\begin{array}{llll}1.23 & 1.46 & 1.72 & 2.02\end{array}$

$\begin{array}{llll}1.26 & 1.50 & 1.79 & 2.12\end{array}$

$\begin{array}{llll}1.30 & 1.56 & 1.88 & 2.25\end{array}$

$\begin{array}{llll}1.34 & 1.62 & 1.97 & 2.38\end{array}$

$\begin{array}{llll}1.38 & 1.70 & 2.08 & 2.54\end{array}$

$\begin{array}{llll}1.42 & 1.76 & 2.18 & 2.69\end{array}$

$\begin{array}{llll}1.46 & 1.82 & 2.28 & 2.84\end{array}$

$1.50 \quad 1.90 \quad 2.39 \quad 3.01$

$\begin{array}{llll}1.50 & 1.90 & 2.39 & 3.01\end{array}$

$\begin{array}{llll}1.54 & 1.96 & 2.50 & 3.17 \\ 1.57 & 2.02 & 2.60 & 3.33\end{array}$

$\begin{array}{llll}1.61 & 2.09 & 2.71 & 3.51\end{array}$

$\begin{array}{llll}1.65 & 2.16 & 2.83 & 3.71\end{array}$

$\begin{array}{llll}1.69 & 2.24 & 2.96 & 3.91\end{array}$

$\begin{array}{llll}1.73 & 2.31 & 3.09 & 4.12\end{array}$ 
Table S-1, continued. Projected fuel price indices with assumed general price inflation rates of $2 \%, 3 \%, 4 \%$, and $5 \%$, by end-use sector and fuel type.

Census Region 1 (Connecticut, Maine, Massachusetts, New Hampshire,

New Jersey, New York, Pennsylvania, Rhode Island, Vermont)

Projected April 1 Fuel Price Indices (April 1, $2007=1.00$ )

\begin{tabular}{lcccc}
\multicolumn{5}{c}{ Electricity } \\
Year & $2 \%$ & $3 \%$ & $4 \%$ & $5 \%$ \\
2008 & 1.01 & 1.02 & 1.03 & 1.04 \\
2009 & 0.99 & 1.01 & 1.03 & 1.05 \\
2010 & 0.94 & 0.96 & 0.99 & 1.02 \\
2011 & 0.89 & 0.92 & 0.96 & 0.99 \\
2012 & 0.86 & 0.91 & 0.95 & 1.00 \\
2013 & 0.87 & 0.92 & 0.97 & 1.03 \\
2014 & 0.87 & 0.93 & 0.99 & 1.06 \\
2015 & 0.89 & 0.96 & 1.04 & 1.12 \\
2016 & 0.91 & 0.99 & 1.08 & 1.18 \\
2017 & 0.93 & 1.03 & 1.13 & 1.25 \\
2018 & 0.97 & 1.08 & 1.20 & 1.33 \\
2019 & 0.99 & 1.11 & 1.25 & 1.40 \\
2020 & 1.01 & 1.14 & 1.30 & 1.47 \\
2021 & 1.03 & 1.18 & 1.35 & 1.55 \\
2022 & 1.05 & 1.22 & 1.41 & 1.62 \\
2023 & 1.08 & 1.26 & 1.47 & 1.72 \\
2024 & 1.11 & 1.31 & 1.54 & 1.82 \\
2025 & 1.14 & 1.36 & 1.61 & 1.92 \\
2026 & 1.16 & 1.39 & 1.68 & 2.01 \\
2027 & 1.18 & 1.43 & 1.74 & 2.10 \\
2028 & 1.21 & 1.48 & 1.82 & 2.22 \\
2029 & 1.24 & 1.54 & 1.90 & 2.34 \\
2030 & 1.26 & 1.58 & 1.97 & 2.45 \\
2031 & 1.28 & 1.62 & 2.05 & 2.58 \\
2032 & 1.31 & 1.68 & 2.13 & 2.71 \\
2033 & 1.34 & 1.73 & 2.23 & 2.85 \\
2034 & 1.37 & 1.79 & 2.32 & 3.00 \\
2035 & 1.40 & 1.84 & 2.42 & 3.16 \\
2036 & 1.44 & 1.90 & 2.52 & 3.33 \\
2037 & 1.47 & 1.97 & 2.63 & 3.50
\end{tabular}

Distillate 0il

Inflation Rate

$2 \% \quad 3 \% \quad 4 \% \quad 5 \%$

$0.98 \quad 0.99 \quad 1.00 \quad 1.00$

$0.93 \quad 0.95 \quad 0.97 \quad 0.99$

$0.91 \quad 0.93 \quad 0.96 \quad 0.99$

$0.89 \quad 0.93 \quad 0.96 \quad 1.00$

$0.87 \quad 0.91 \quad 0.96 \quad 1.01$

$0.86 \quad 0.91 \quad 0.96 \quad 1.02$

$\begin{array}{llll}0.86 & 0.92 & 0.98 & 1.05\end{array}$

$\begin{array}{llll}0.87 & 0.94 & 1.02 & 1.10\end{array}$

$\begin{array}{llll}0.87 & 0.94 & 1.02 & 1.10\end{array}$

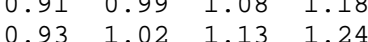

$\begin{array}{cccc}\odot .93 & 1.02 & 1.13 & 1.24 \\ 0.95 & 1.06 & 1.18 & 1.31\end{array}$

$\begin{array}{llll}0.99 & 1.12 & 1.25 & 1.41\end{array}$

$\begin{array}{llll}1.02 & 1.16 & 1.32 & 1.49\end{array}$

$\begin{array}{llll}1.05 & 1.21 & 1.38 & 1.58\end{array}$

$\begin{array}{llll}1.10 & 1.27 & 1.47 & 1.69\end{array}$

$1.10 \quad 1.29 \quad 1.51 \quad 1.76$

$\begin{array}{llll}1.13 & 1.34 & 1.58 & 1.85\end{array}$

$\begin{array}{llll}1.13 & 1.34 & 1.58 & 1.85 \\ 1.18 & 1.40 & 1.67 & 1.99\end{array}$

$\begin{array}{llll}1.18 & 1.40 & 1.67 & 1.99 \\ 1.21 & 1.45 & 1.74 & 2.09\end{array}$

$\begin{array}{llll}1.24 & 1.51 & 1.84 & 2.22\end{array}$

$\begin{array}{llll}1.30 & 1.59 & 1.95 & 2.38\end{array}$

$\begin{array}{llll}1.33 & 1.65 & 2.04 & 2.52\end{array}$

$\begin{array}{llll}1.37 & 1.72 & 2.14 & 2.67\end{array}$

$\begin{array}{llll}1.40 & 1.78 & 2.24 & 2.82\end{array}$

$\begin{array}{llll}1.43 & 1.83 & 2.33 & 2.96\end{array}$

$\begin{array}{llll}1.46 & 1.89 & 2.43 & 3.11\end{array}$

$\begin{array}{llll}1.50 & 1.95 & 2.53 & 3.27\end{array}$

$\begin{array}{llll}1.53 & 2.01 & 2.63 & 3.44\end{array}$

$\begin{array}{llll}1.56 & 2.07 & 2.74 & 3.61\end{array}$
Residual 0il

\section{Inflation Rate}

$2 \% 3 \% \quad 4 \% 5 \%$

$\begin{array}{llll}0.97 & 0.98 & 0.99 & 1.00\end{array}$

$\begin{array}{llll}0.98 & 1.00 & 1.02 & 1.04\end{array}$

$\begin{array}{llll}0.99 & 1.02 & 1.05 & 1.08\end{array}$

$\begin{array}{llll}0.96 & 1.00 & 1.04 & 1.08\end{array}$

$0.96 \quad 1.00 \quad 1.05 \quad 1.11$

$\begin{array}{lllll}0.87 & 0.93 & 0.98 & 1.04\end{array}$

$\begin{array}{llll}0.89 & 0.96 & 1.03 & 1.10\end{array}$

$\begin{array}{llll}0.94 & 1.02 & 1.10 & 1.19\end{array}$

$\begin{array}{llll}0.97 & 1.06 & 1.15 & 1.25\end{array}$

$\begin{array}{llll}1.00 & 1.10 & 1.21 & 1.33\end{array}$

$\begin{array}{llll}1.04 & 1.15 & 1.28 & 1.43\end{array}$

$\begin{array}{llll}1.09 & 1.23 & 1.38 & 1.55\end{array}$

$\begin{array}{llll}1.14 & 1.29 & 1.46 & 1.66\end{array}$

$\begin{array}{llll}1.18 & 1.35 & 1.55 & 1.77\end{array}$

$\begin{array}{llll}1.23 & 1.43 & 1.65 & 1.90\end{array}$

$\begin{array}{llll}1.24 & 1.45 & 1.69 & 1.98\end{array}$

$\begin{array}{llll}1.28 & 1.51 & 1.78 & 2.09\end{array}$

$\begin{array}{llll}1.28 & 1.52 & 1.81 & 2.16\end{array}$

$\begin{array}{llll}1.31 & 1.58 & 1.90 & 2.28\end{array}$

$\begin{array}{llll}1.35 & 1.65 & 2.00 & 2.42\end{array}$

$\begin{array}{llll}1.40 & 1.71 & 2.10 & 2.57\end{array}$

$\begin{array}{llll}1.39 & 1.73 & 2.13 & 2.63\end{array}$

$\begin{array}{llll}1.42 & 1.77 & 2.21 & 2.76\end{array}$

$\begin{array}{llll}1.45 & 1.83 & 2.31 & 2.91\end{array}$

$\begin{array}{llll}1.48 & 1.89 & 2.41 & 3.06\end{array}$

$\begin{array}{llll}1.51 & 1.95 & 2.51 & 3.22\end{array}$

$\begin{array}{llll}1.55 & 2.02 & 2.62 & 3.39\end{array}$

$\begin{array}{llll}1.58 & 2.08 & 2.72 & 3.56\end{array}$

$\begin{array}{llll}1.62 & 2.15 & 2.84 & 3.75\end{array}$

$\begin{array}{llll}1.65 & 2.21 & 2.96 & 3.94\end{array}$
Natural Gas

Inflation Rate

$2 \%$

$\begin{array}{llll}0.99 & 1.00 & 1.01 & 1.02\end{array}$

$\begin{array}{llll}0.95 & 0.97 & 0.99 & 1.01\end{array}$

$\begin{array}{llll}0.92 & 0.95 & 0.98 & 1.01\end{array}$

$\begin{array}{llll}0.89 & 0.93 & 0.96 & 1.00\end{array}$

$\begin{array}{llll}0.88 & 0.92 & 0.97 & 1.01\end{array}$

$\begin{array}{llll}0.86 & 0.92 & 0.97 & 1.03\end{array}$

$\begin{array}{llll}0.88 & 0.94 & 1.00 & 1.07\end{array}$

$\begin{array}{llll}0.89 & 0.96 & 1.03 & 1.12\end{array}$

$\begin{array}{llll}0.90 & 0.99 & 1.08 & 1.17\end{array}$

$\begin{array}{llll}0.95 & 1.04 & 1.15 & 1.27\end{array}$

$0.97 \quad 1.08 \quad 1.20 \quad 1.33$

$0.98 \quad 1.10 \quad 1.24 \quad 1.39$

$\begin{array}{llll}1.01 & 1.14 & 1.30 & 1.47\end{array}$

$\begin{array}{llll}1.02 & 1.17 & 1.34 & 1.53\end{array}$

$\begin{array}{llll}1.05 & 1.22 & 1.41 & 1.63\end{array}$

$\begin{array}{llll}1.09 & 1.28 & 1.49 & 1.74\end{array}$

$\begin{array}{llll}1.13 & 1.33 & 1.57 & 1.85\end{array}$

$\begin{array}{llll}1.15 & 1.37 & 1.63 & 1.94\end{array}$

$\begin{array}{llll}1.18 & 1.42 & 1.71 & 2.05\end{array}$

$\begin{array}{llll}1.22 & 1.48 & 1.79 & 2.17\end{array}$

$\begin{array}{llll}1.26 & 1.55 & 1.90 & 2.32\end{array}$

$\begin{array}{llll}1.30 & 1.61 & 1.99 & 2.46\end{array}$

$\begin{array}{llll}1.33 & 1.67 & 2.08 & 2.60\end{array}$

$\begin{array}{llll}1.37 & 1.73 & 2.19 & 2.75\end{array}$

$\begin{array}{llll}1.41 & 1.81 & 2.30 & 2.92\end{array}$

$\begin{array}{llll}1.46 & 1.88 & 2.42 & 3.10\end{array}$

$\begin{array}{llll}1.50 & 1.96 & 2.54 & 3.29\end{array}$

$\begin{array}{llll}1.55 & 2.04 & 2.67 & 3.49\end{array}$

$\begin{array}{llll}1.60 & 2.12 & 2.81 & 3.71\end{array}$

$\begin{array}{llll}1.65 & 2.21 & 2.95 & 3.94\end{array}$
Coal

Inflation Rate

$2 \% 3 \% \quad 4 \% 5 \%$

$\begin{array}{llll}1.03 & 1.04 & 1.05 & 1.06\end{array}$

$\begin{array}{llll}1.05 & 1.07 & 1.09 & 1.11\end{array}$

$\begin{array}{llll}1.08 & 1.12 & 1.15 & 1.18\end{array}$

$\begin{array}{llll}1.11 & 1.16 & 1.20 & 1.25\end{array}$

$\begin{array}{llll}1.11 & 1.16 & 1.22 & 1.28\end{array}$

$\begin{array}{llll}1.11 & 1.18 & 1.25 & 1.32\end{array}$

$\begin{array}{llll}1.11 & 1.19 & 1.27 & 1.36\end{array}$

$\begin{array}{llll}1.12 & 1.22 & 1.31 & 1.42\end{array}$

$\begin{array}{llll}1.14 & 1.24 & 1.35 & 1.48\end{array}$

$\begin{array}{llll}1.15 & 1.27 & 1.39 & 1.53\end{array}$

$\begin{array}{llll}1.15 & 1.29 & 1.43 & 1.59\end{array}$

$\begin{array}{llll}1.17 & 1.31 & 1.47 & 1.65\end{array}$

$\begin{array}{llll}1.20 & 1.36 & 1.54 & 1.74\end{array}$

$\begin{array}{llll}1.23 & 1.41 & 1.61 & 1.84\end{array}$

$\begin{array}{llll}1.26 & 1.45 & 1.68 & 1.94\end{array}$

$\begin{array}{llll}1.29 & 1.50 & 1.76 & 2.05\end{array}$

$\begin{array}{llll}1.33 & 1.56 & 1.84 & 2.17\end{array}$

$\begin{array}{llll}1.35 & 1.61 & 1.92 & 2.28\end{array}$

$\begin{array}{llll}1.39 & 1.67 & 2.00 & 2.40\end{array}$

$\begin{array}{llll}1.43 & 1.73 & 2.10 & 2.55\end{array}$

$\begin{array}{llll}1.47 & 1.80 & 2.21 & 2.70\end{array}$

$\begin{array}{llll}1.50 & 1.87 & 2.31 & 2.85\end{array}$

$\begin{array}{llll}1.54 & 1.93 & 2.41 & 3.00\end{array}$

$\begin{array}{lllll}1.59 & 2.01 & 2.53 & 3.18\end{array}$

$\begin{array}{llll}1.63 & 2.08 & 2.64 & 3.36\end{array}$

$\begin{array}{llll}1.66 & 2.14 & 2.75 & 3.52\end{array}$

$\begin{array}{llll}1.70 & 2.21 & 2.87 & 3.72\end{array}$

$\begin{array}{llll}1.74 & 2.29 & 3.00 & 3.92\end{array}$

$\begin{array}{llll}1.78 & 2.37 & 3.13 & 4.13 \\ 1.83 & 2.45 & 3.27 & 4.36\end{array}$ 


\section{Table S-2. Projected fuel price indices with assumed general price inflation rates}

of $2 \%, 3 \%, 4 \%$, and $5 \%$, by end-use sector and fuel type.

Census Region 2 (Illinois, Indiana, Iowa, Kansas, Michigan, Minnesota, Missouri, Nebraska, North Dakota, Ohio, South Dakota, Wisconsin)

Projected April 1 Fuel Price Indices (April 1, $2007=1.00$ )

Electricity

Inflation Rate $2 \% \quad 3 \% \quad 4 \% \quad 5 \%$

$1.03 \quad 1.04 \quad 1.05 \quad 1.06$

$1.06 \quad 1.08 \quad 1.10 \quad 1.12$

$\begin{array}{llll}1.08 & 1.12 & 1.15 & 1.18\end{array}$

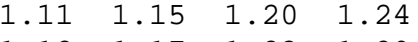

$1.12 \quad 1.17 \quad 1.23 \quad 1.29$

$1.13 \quad 1.20 \quad 1.27 \quad 1.35$

$\begin{array}{llll}1.15 & 1.24 & 1.32 & 1.41\end{array}$

$\begin{array}{llll}1.17 & 1.27 & 1.37 & 1.48\end{array}$

$1.20 \quad 1.31 \quad 1.43 \quad 1.56$

$\begin{array}{llll}1.23 & 1.36 & 1.49 & 1.64\end{array}$

$\begin{array}{llll}1.26 & 1.41 & 1.56 & 1.74\end{array}$

$\begin{array}{llll}1.29 & 1.45 & 1.63 & 1.82\end{array}$

$\begin{array}{llll}1.31 & 1.49 & 1.69 & 1.91\end{array}$

$\begin{array}{llll}1.34 & 1.54 & 1.76 & 2.02\end{array}$

$\begin{array}{llll}1.37 & 1.59 & 1.83 & 2.12\end{array}$

$\begin{array}{llll}1.40 & 1.64 & 1.91 & 2.23\end{array}$

$\begin{array}{llll}1.43 & 1.69 & 1.99 & 2.35\end{array}$

$\begin{array}{llll}1.46 & 1.74 & 2.07 & 2.46\end{array}$

$\begin{array}{llll}1.48 & 1.78 & 2.14 & 2.57\end{array}$

$\begin{array}{llll}1.51 & 1.83 & 2.22 & 2.69\end{array}$

$\begin{array}{llll}1.54 & 1.89 & 2.32 & 2.83\end{array}$

$\begin{array}{llll}1.58 & 1.95 & 2.42 & 2.98\end{array}$

$\begin{array}{llll}1.61 & 2.01 & 2.52 & 3.13\end{array}$

$\begin{array}{llll}1.64 & 2.08 & 2.62 & 3.29\end{array}$

$\begin{array}{llll}1.68 & 2.14 & 2.72 & 3.46\end{array}$

$\begin{array}{llll}1.71 & 2.20 & 2.83 & 3.63\end{array}$

$\begin{array}{llll}1.74 & 2.27 & 2.95 & 3.82\end{array}$

$\begin{array}{llll}1.78 & 2.34 & 3.07 & 4.01\end{array}$

$\begin{array}{llll}1.82 & 2.41 & 3.19 & 4.21\end{array}$

$\begin{array}{llll}1.85 & 2.48 & 3.32 & 4.42\end{array}$
Distillate 0il

LPG

\begin{tabular}{cccc}
\multicolumn{4}{c}{ Inflation Rate } \\
1.00 & 3.01 & $4 \%$ & $5 \%$ \\
0.96 & 0.98 & 1.02 & 1.03 \\
0.92 & 0.94 & 0.97 & 1.02 \\
0.87 & 0.90 & 0.94 & 0.97 \\
0.82 & 0.86 & 0.91 & 0.95 \\
0.79 & 0.84 & 0.89 & 0.94 \\
0.78 & 0.84 & 0.90 & 0.96 \\
0.80 & 0.86 & 0.93 & 1.01 \\
0.81 & 0.89 & 0.97 & 1.06 \\
0.84 & 0.92 & 1.02 & 1.12 \\
0.86 & 0.96 & 1.07 & 1.19 \\
0.90 & 1.01 & 1.13 & 1.27 \\
0.92 & 1.05 & 1.19 & 1.35 \\
0.95 & 1.09 & 1.25 & 1.43 \\
0.98 & 1.14 & 1.31 & 1.52 \\
1.00 & 1.17 & 1.37 & 1.59 \\
1.03 & 1.21 & 1.43 & 1.68 \\
1.05 & 1.26 & 1.49 & 1.78 \\
1.08 & 1.30 & 1.56 & 1.87 \\
1.11 & 1.35 & 1.64 & 1.98 \\
1.14 & 1.40 & 1.71 & 2.10 \\
1.18 & 1.46 & 1.81 & 2.23 \\
1.21 & 1.51 & 1.89 & 2.36 \\
1.24 & 1.56 & 1.97 & 2.48 \\
1.26 & 1.61 & 2.05 & 2.61 \\
1.29 & 1.66 & 2.13 & 2.74 \\
1.32 & 1.71 & 2.22 & 2.88 \\
1.34 & 1.77 & 2.31 & 3.03 \\
1.37 & 1.82 & 2.41 & 3.18 \\
1.40 & 1.88 & 2.51 & 3.34
\end{tabular}

Natural Gas

\begin{tabular}{rrrr}
\multicolumn{4}{c}{ Inflation Rate } \\
1.07 & 3.08 & $4 \%$ & $5 \%$ \\
1.07 & 1.09 & 1.09 & 1.10 \\
1.08 & 1.11 & 1.14 & 1.14 \\
1.08 & 1.12 & 1.17 & 1.21 \\
1.09 & 1.15 & 1.20 & 1.26 \\
1.10 & 1.17 & 1.24 & 1.31 \\
1.12 & 1.20 & 1.29 & 1.37 \\
1.14 & 1.24 & 1.33 & 1.44 \\
1.17 & 1.28 & 1.39 & 1.52 \\
1.20 & 1.33 & 1.46 & 1.61 \\
1.23 & 1.37 & 1.52 & 1.69 \\
1.25 & 1.40 & 1.58 & 1.77 \\
1.28 & 1.45 & 1.64 & 1.86 \\
1.30 & 1.49 & 1.71 & 1.95 \\
1.33 & 1.54 & 1.79 & 2.06 \\
1.37 & 1.60 & 1.87 & 2.18 \\
1.40 & 1.66 & 1.95 & 2.30 \\
1.43 & 1.71 & 2.03 & 2.42 \\
1.46 & 1.76 & 2.12 & 2.54 \\
1.50 & 1.82 & 2.21 & 2.67 \\
1.54 & 1.89 & 2.31 & 2.82 \\
1.57 & 1.95 & 2.41 & 2.98 \\
1.61 & 2.01 & 2.52 & 3.14 \\
1.64 & 2.08 & 2.62 & 3.30 \\
1.68 & 2.14 & 2.72 & 3.46 \\
1.71 & 2.21 & 2.84 & 3.64 \\
1.75 & 2.27 & 2.95 & 3.82 \\
1.78 & 2.34 & 3.07 & 4.01 \\
1.82 & 2.42 & 3.20 & 4.22 \\
1.86 & 2.49 & 3.33 & 4.43
\end{tabular}

Inflation Rate

$2 \% \quad 3 \% \quad 4 \% \quad 5 \%$

$0.99 \quad 1.00 \quad 1.01 \quad 1.02$

$\begin{array}{llll}0.97 & 0.99 & 1.01 & 1.03\end{array}$

$\begin{array}{llll}0.96 & 0.99 & 1.02 & 1.05\end{array}$

$0.94 \quad 0.98 \quad 1.02 \quad 1.06$

$\begin{array}{llll}0.94 & 0.99 & 1.04 & 1.09\end{array}$

$\begin{array}{llll}0.94 & 1.00 & 1.06 & 1.12\end{array}$

$\begin{array}{llll}0.96 & 1.03 & 1.10 & 1.18\end{array}$

$\begin{array}{llll}0.98 & 1.06 & 1.14 & 1.23\end{array}$

$\begin{array}{llll}1.00 & 1.09 & 1.19 & 1.30\end{array}$

$\begin{array}{llll}1.04 & 1.15 & 1.27 & 1.39\end{array}$

$\begin{array}{llll}1.07 & 1.19 & 1.32 & 1.47\end{array}$

$\begin{array}{llll}1.09 & 1.22 & 1.37 & 1.54\end{array}$

$\begin{array}{llll}1.12 & 1.27 & 1.44 & 1.63\end{array}$

$\begin{array}{llll}1.14 & 1.31 & 1.50 & 1.71\end{array}$

$\begin{array}{llll}1.17 & 1.36 & 1.57 & 1.81\end{array}$

$\begin{array}{llll}1.22 & 1.42 & 1.66 & 1.93\end{array}$

$\begin{array}{llll}1.26 & 1.48 & 1.75 & 2.06\end{array}$

$\begin{array}{llll}1.29 & 1.53 & 1.83 & 2.17\end{array}$

$\begin{array}{llll}1.32 & 1.59 & 1.91 & 2.29\end{array}$

$\begin{array}{llll}1.36 & 1.65 & 2.00 & 2.42\end{array}$

$\begin{array}{llll}1.40 & 1.72 & 2.10 & 2.57\end{array}$

$\begin{array}{llll}1.44 & 1.79 & 2.21 & 2.73\end{array}$

$\begin{array}{llll}1.48 & 1.85 & 2.32 & 2.89\end{array}$

$\begin{array}{llll}1.52 & 1.92 & 2.42 & 3.05\end{array}$

$\begin{array}{llll}1.56 & 2.00 & 2.54 & 3.23\end{array}$

$\begin{array}{llll}1.61 & 2.07 & 2.66 & 3.41\end{array}$

$\begin{array}{llll}1.65 & 2.15 & 2.79 & 3.61\end{array}$

$\begin{array}{llll}1.70 & 2.23 & 2.92 & 3.82\end{array}$

$\begin{array}{llll}1.74 & 2.31 & 3.06 & 4.04\end{array}$

$\begin{array}{llll}1.79 & 2.40 & 3.21 & 4.28\end{array}$ 
Table S-2, continued. Projected fuel price indices with assumed general price inflation rates of $2 \%, 3 \%, 4 \%$, and $5 \%$, by end-use sector and fuel type.

Census Region 2 (Illinois, Indiana, Iowa, Kansas, Michigan, Minnesota,

Missouri, Nebraska, North Dakota, Ohio, South Dakota, Wisconsin)

Projected April 1 Fuel Price Indices (April 1, $2007=1.00$ )

\begin{tabular}{lrccc}
\multicolumn{4}{c}{ Electricity } \\
Year & $2 \%$ Inflation Rate \\
2008 & 1.03 & 1.04 & 4.06 & 1.07 \\
2009 & 1.06 & 1.08 & 1.11 & 1.13 \\
2010 & 1.09 & 1.12 & 1.15 & 1.19 \\
2011 & 1.10 & 1.15 & 1.19 & 1.24 \\
2012 & 1.10 & 1.16 & 1.21 & 1.27 \\
2013 & 1.11 & 1.18 & 1.25 & 1.32 \\
2014 & 1.12 & 1.20 & 1.29 & 1.38 \\
2015 & 1.14 & 1.24 & 1.33 & 1.44 \\
2016 & 1.17 & 1.28 & 1.40 & 1.52 \\
2017 & 1.21 & 1.34 & 1.47 & 1.62 \\
2018 & 1.25 & 1.39 & 1.55 & 1.72 \\
2019 & 1.28 & 1.44 & 1.62 & 1.81 \\
2020 & 1.31 & 1.49 & 1.69 & 1.91 \\
2021 & 1.35 & 1.54 & 1.77 & 2.02 \\
2022 & 1.38 & 1.60 & 1.85 & 2.13 \\
2023 & 1.42 & 1.65 & 1.93 & 2.25 \\
2024 & 1.46 & 1.72 & 2.03 & 2.38 \\
2025 & 1.49 & 1.77 & 2.11 & 2.51 \\
2026 & 1.51 & 1.82 & 2.18 & 2.62 \\
2027 & 1.54 & 1.87 & 2.26 & 2.74 \\
2028 & 1.57 & 1.92 & 2.36 & 2.88 \\
2029 & 1.60 & 1.99 & 2.46 & 3.03 \\
2030 & 1.64 & 2.05 & 2.56 & 3.19 \\
2031 & 1.67 & 2.11 & 2.66 & 3.35 \\
2032 & 1.71 & 2.18 & 2.77 & 3.52 \\
2033 & 1.74 & 2.25 & 2.89 & 3.71 \\
2034 & 1.78 & 2.32 & 3.01 & 3.90 \\
2035 & 1.82 & 2.39 & 3.14 & 4.10 \\
2036 & 1.86 & 2.47 & 3.27 & 4.32 \\
2037 & 1.90 & 2.55 & 3.41 & 4.54
\end{tabular}

Distillate 0il

Inflation Rate

$2 \% \quad 3 \% \quad 4 \% 5 \%$

$0.98 \quad 0.99 \quad 1.00 \quad 1.01$

$\begin{array}{llll}0.93 & 0.95 & 0.97 & 0.99\end{array}$

$\begin{array}{llll}0.89 & 0.92 & 0.95 & 0.98\end{array}$

$\begin{array}{llll}0.85 & 0.89 & 0.92 & 0.96\end{array}$

$0.82 \quad 0.86 \quad 0.90 \quad 0.94$

$\begin{array}{llll}0.79 & 0.84 & 0.89 & 0.95\end{array}$

$\begin{array}{llll}0.79 & 0.84 & 0.90 & 0.97\end{array}$

$0.80 \quad 0.87 \quad 0.94 \quad 1.01$

$0.83 \quad 0.90 \quad 0.98 \quad 1.07$

$\begin{array}{llll}0.85 & 0.94 & 1.03 & 1.13\end{array}$

$0.87 \quad 0.97 \quad 1.08 \quad 1.20$

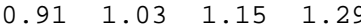

$\begin{array}{llll}0.94 & 1.06 & 1.21 & 1.37\end{array}$

$\begin{array}{llll}0.96 & 1.11 & 1.27 & 1.45\end{array}$

$\begin{array}{llll}1.00 & 1.16 & 1.34 & 1.55\end{array}$

$\begin{array}{llll}1.01 & 1.18 & 1.38 & 1.61\end{array}$

$\begin{array}{llll}1.04 & 1.23 & 1.45 & 1.71\end{array}$

$\begin{array}{llll}1.08 & 1.28 & 1.53 & 1.81\end{array}$

$\begin{array}{llll}1.10 & 1.33 & 1.59 & 1.91\end{array}$

$\begin{array}{llll}1.14 & 1.38 & 1.68 & 2.03\end{array}$

$\begin{array}{llll}1.18 & 1.45 & 1.77 & 2.17\end{array}$

$\begin{array}{llll}1.21 & 1.50 & 1.86 & 2.30\end{array}$

$\begin{array}{llll}1.25 & 1.56 & 1.95 & 2.43\end{array}$

$\begin{array}{llll}1.28 & 1.62 & 2.04 & 2.56\end{array}$

$\begin{array}{llll}1.31 & 1.67 & 2.12 & 2.69\end{array}$

$\begin{array}{llll}1.31 & 1.67 & 2.12 & 2.69 \\ 1.33 & 1.72 & 2.21 & 2.83\end{array}$

$\begin{array}{llll}1.36 & 1.77 & 2.30 & 2.98\end{array}$

$\begin{array}{llll}1.39 & 1.83 & 2.39 & 3.13\end{array}$

$\begin{array}{llll}1.42 & 1.88 & 2.49 & 3.29\end{array}$

$\begin{array}{llll}1.45 & 1.94 & 2.60 & 3.46\end{array}$
- - COMMERCIAL - - -

\begin{tabular}{rrrr}
\multicolumn{4}{c}{ Residual 0il } \\
\multicolumn{4}{c}{ Inflation Rate } \\
$2 \%$ & $3 \%$ & $4 \%$ & $5 \%$ \\
1.11 & 1.12 & 1.13 & 1.15 \\
1.10 & 1.12 & 1.14 & 1.16 \\
1.06 & 1.09 & 1.12 & 1.16 \\
1.04 & 1.08 & 1.12 & 1.16 \\
1.02 & 1.07 & 1.12 & 1.17 \\
1.01 & 1.07 & 1.13 & 1.20 \\
1.01 & 1.08 & 1.15 & 1.23 \\
1.03 & 1.11 & 1.20 & 1.29 \\
1.05 & 1.14 & 1.25 & 1.36 \\
1.08 & 1.19 & 1.31 & 1.45 \\
1.11 & 1.24 & 1.37 & 1.53 \\
1.15 & 1.29 & 1.45 & 1.63 \\
1.18 & 1.34 & 1.52 & 1.73 \\
1.22 & 1.40 & 1.61 & 1.84 \\
1.27 & 1.47 & 1.69 & 1.96 \\
1.29 & 1.50 & 1.76 & 2.05 \\
1.32 & 1.56 & 1.84 & 2.17 \\
1.36 & 1.62 & 1.93 & 2.29 \\
1.39 & 1.68 & 2.02 & 2.42 \\
1.43 & 1.74 & 2.11 & 2.55 \\
1.47 & 1.80 & 2.20 & 2.69 \\
1.51 & 1.87 & 2.32 & 2.86 \\
1.55 & 1.94 & 2.43 & 3.02 \\
1.59 & 2.00 & 2.53 & 3.18 \\
1.62 & 2.07 & 2.63 & 3.35 \\
1.65 & 2.13 & 2.74 & 3.52 \\
1.69 & 2.20 & 2.86 & 3.70 \\
1.73 & 2.27 & 2.97 & 3.89 \\
1.76 & 2.34 & 3.10 & 4.09 \\
1.80 & 2.41 & 3.23 & 4.30
\end{tabular}

Coal

Inflation Rate

$\begin{array}{rrrr}2 \% & 3 \% & 4 \% & 5 \% \\ 1.03 & 1.04 & 1.05 & 1.06\end{array}$

$\begin{array}{llll}1.03 & 1.04 & 1.05 & 1.06 \\ 1.06 & 1.08 & 1.10 & 1.12\end{array}$

$\begin{array}{llll}1.11 & 1.14 & 1.18 & 1.21\end{array}$

$\begin{array}{llll}1.15 & 1.19 & 1.24 & 1.29\end{array}$

$\begin{array}{llll}1.17 & 1.23 & 1.29 & 1.35\end{array}$

$\begin{array}{llll}1.19 & 1.26 & 1.34 & 1.41\end{array}$

$\begin{array}{lllll}1.21 & 1.30 & 1.39 & 1.49\end{array}$

$\begin{array}{llll}1.21 & 1.30 & 1.39 & 1.49\end{array}$

$\begin{array}{llll}1.23 & 1.33 & 1.43 & 1.55 \\ 1.24 & 1.36 & 1.48 & 1.62\end{array}$

$\begin{array}{llll}1.24 & 1.36 & 1.48 & 1.62 \\ 1.26 & 1.39 & 1.53 & 1.68\end{array}$

$\begin{array}{llll}1.27 & 1.42 & 1.58 & 1.75\end{array}$

$\begin{array}{llll}1.30 & 1.46 & 1.64 & 1.84\end{array}$

$\begin{array}{llll}1.32 & 1.50 & 1.70 & 1.93\end{array}$

$\begin{array}{llll}1.35 & 1.55 & 1.77 & 2.03\end{array}$

$\begin{array}{llll}1.37 & 1.59 & 1.83 & 2.12\end{array}$

$1.40 \quad 1.64 \quad 1.92 \quad 2.23$

$\begin{array}{llll}1.44 & 1.70 & 2.00 & 2.36\end{array}$

$\begin{array}{llll}1.47 & 1.76 & 2.09 & 2.48\end{array}$

$\begin{array}{llll}1.52 & 1.83 & 2.19 & 2.63\end{array}$

$\begin{array}{llll}1.55 & 1.89 & 2.29 & 2.78\end{array}$

$\begin{array}{llll}1.59 & 1.96 & 2.39 & 2.93\end{array}$

$\begin{array}{llll}1.64 & 2.03 & 2.51 & 3.10\end{array}$

$\begin{array}{lllll}1.67 & 2.09 & 2.61 & 3.26\end{array}$

$\begin{array}{llll}1.71 & 2.016 & 2.73 & 3.43\end{array}$

$\begin{array}{llll}1.71 & 2.16 & 2.73 & 3.43\end{array}$

$\begin{array}{llll}1.75 & 2.24 & 2.85 & 3.62 \\ 1.80 & 2.32 & 2.98 & 3.82\end{array}$

$\begin{array}{llll}1.84 & 2.40 & 3.11 & 4.03\end{array}$

$\begin{array}{llll}1.89 & 2.48 & 3.25 & 4.25\end{array}$

$\begin{array}{llll}1.93 & 2.56 & 3.39 & 4.48\end{array}$

$\begin{array}{llll}1.98 & 2.65 & 3.54 & 4.72\end{array}$ 
Table S-2, continued. Projected fuel price indices with assumed general price inflation rates of $2 \%, 3 \%, 4 \%$, and $5 \%$, by end-use sector and fuel type.

Census Region 2 (Illinois, Indiana, Iowa, Kansas, Michigan, Minnesota,

Missouri, Nebraska, North Dakota, Ohio, South Dakota, Wisconsin)

Projected April 1 Fuel Price Indices (April 1, $2007=1.00$ )

\begin{tabular}{|c|c|c|c|c|c|c|c|c|c|c|c|c|c|c|c|c|c|c|c|c|}
\hline \multirow[b]{3}{*}{ Year } & \multicolumn{4}{|c|}{ Electricity } & \multicolumn{4}{|c|}{ Distillate $0 i l$} & \multicolumn{4}{|c|}{ Residual 0il } & \multicolumn{4}{|c|}{ Natural Gas } & \multicolumn{4}{|c|}{ Coal } \\
\hline & \multicolumn{4}{|c|}{ Inflation Rate } & \multicolumn{4}{|c|}{ Inflation Rate } & \multicolumn{4}{|c|}{ Inflation Rate } & \multicolumn{4}{|c|}{ Inflation Rate } & \multicolumn{4}{|c|}{ Inflation Rate } \\
\hline & $2 \%$ & $3 \%$ & $4 \%$ & $5 \%$ & $2 \%$ & $3 \%$ & $4 \%$ & $5 \%$ & $2 \%$ & $3 \%$ & $4 \%$ & $5 \%$ & $2 \%$ & $3 \%$ & $4 \%$ & $5 \%$ & $2 \%$ & $3 \%$ & $4 \%$ & $5 \%$ \\
\hline 2008 & 1.05 & 1.07 & 1.08 & 1.09 & 0.97 & 0.98 & 0.98 & 0.99 & 1.11 & 1.12 & 1.13 & 1.14 & 1.02 & 1.03 & 1.04 & 1.05 & 1.03 & 1.04 & 1.05 & 1.06 \\
\hline 2009 & 1.08 & 1.10 & 1.12 & 1.14 & 0.92 & 0.94 & 0.96 & 0.98 & 1.09 & 1.11 & 1.13 & 1.16 & 0.99 & 1.01 & 1.02 & 1.04 & 1.05 & 1.07 & 1.09 & 1.11 \\
\hline 2010 & 1.08 & 1.11 & 1.14 & 1.18 & 0.90 & 0.93 & 0.96 & 0.98 & 1.06 & 1.09 & 1.12 & 1.15 & 0.96 & 0.99 & 1.02 & 1.05 & 1.10 & 1.13 & 1.17 & 1.20 \\
\hline 2011 & 1.06 & 1.10 & 1.15 & 1.19 & 0.89 & 0.92 & $\odot .96$ & 1.00 & 1.03 & 1.07 & 1.11 & 1.16 & 0.93 & 0.97 & 1.01 & 1.05 & 1.13 & 1.18 & 1.22 & 1.27 \\
\hline 2012 & 1.04 & 1.09 & 1.15 & 1.20 & 0.87 & 0.91 & 0.96 & 1.00 & 1.01 & 1.06 & 1.11 & 1.17 & 0.92 & 0.97 & 1.02 & 1.07 & 1.15 & 1.21 & 1.27 & 1.33 \\
\hline 2013 & 1.04 & 1.10 & 1.17 & 1.23 & 0.86 & 0.91 & 0.97 & 1.02 & 1.00 & 1.06 & 1.13 & 1.19 & 0.91 & 0.97 & 1.02 & 1.08 & 1.17 & 1.24 & 1.31 & 1.39 \\
\hline 2014 & 1.05 & 1.12 & 1.20 & 1.29 & 0.86 & 0.92 & 0.98 & 1.05 & 1.00 & 1.07 & 1.15 & 1.23 & 0.93 & 0.99 & 1.06 & 1.13 & 1.19 & 1.27 & 1.36 & 1.46 \\
\hline 2015 & 1.07 & 1.15 & 1.25 & 1.34 & 0.87 & 0.94 & 1.02 & 1.10 & 1.02 & 1.10 & 1.19 & 1.29 & 0.94 & 1.01 & 1.10 & 1.18 & 1.20 & 1.30 & 1.40 & 1.52 \\
\hline 2016 & 1.10 & 1.20 & 1.30 & 1.42 & 0.90 & 0.99 & 1.08 & 1.17 & 1.04 & 1.14 & 1.24 & 1.35 & 0.96 & 1.05 & 1.15 & 1.25 & 1.22 & 1.33 & 1.45 & 1.58 \\
\hline 2017 & 1.13 & 1.25 & 1.38 & 1.52 & 0.92 & 1.02 & 1.12 & 1.24 & 1.08 & 1.19 & 1.31 & 1.44 & 1.01 & 1.12 & 1.23 & 1.35 & 1.24 & 1.37 & 1.51 & 1.66 \\
\hline 2018 & 1.17 & 1.31 & 1.45 & 1.61 & 0.95 & 1.06 & 1.17 & 1.30 & 1.10 & 1.23 & 1.37 & 1.52 & 1.03 & 1.15 & 1.28 & 1.42 & 1.27 & 1.41 & 1.57 & 1.75 \\
\hline 2019 & 1.20 & 1.35 & 1.52 & 1.70 & 0.99 & 1.12 & 1.25 & 1.41 & 1.15 & 1.29 & 1.45 & 1.62 & 1.04 & 1.17 & 1.32 & 1.48 & 1.29 & 1.46 & 1.63 & 1.83 \\
\hline 2020 & 1.24 & 1.40 & 1.59 & 1.80 & 1.02 & 1.15 & 1.31 & 1.48 & 1.18 & 1.34 & 1.52 & 1.72 & 1.07 & 1.22 & 1.38 & 1.56 & 1.33 & 1.51 & 1.71 & 1.93 \\
\hline 2021 & 1.27 & 1.45 & 1.66 & 1.90 & 1.04 & 1.20 & 1.37 & 1.56 & 1.22 & 1.40 & 1.60 & 1.83 & 1.09 & 1.26 & 1.44 & 1.64 & 1.36 & 1.56 & 1.79 & 2.04 \\
\hline 2022 & 1.30 & 1.50 & 1.74 & 2.00 & 1.08 & 1.25 & 1.45 & 1.67 & 1.26 & 1.46 & 1.69 & 1.95 & 1.13 & 1.31 & 1.51 & 1.74 & 1.39 & 1.61 & 1.87 & 2.15 \\
\hline 2023 & 1.33 & 1.56 & 1.82 & 2.12 & 1.09 & 1.28 & 1.49 & 1.74 & 1.28 & 1.50 & 1.75 & 2.03 & 1.17 & 1.37 & 1.60 & 1.87 & 1.42 & 1.66 & 1.94 & 2.26 \\
\hline 2024 & 1.37 & 1.62 & 1.91 & 2.24 & 1.12 & 1.32 & 1.56 & 1.84 & 1.32 & 1.55 & 1.83 & 2.16 & 1.22 & 1.43 & 1.69 & 1.99 & 1.46 & 1.72 & 2.03 & 2.39 \\
\hline 2025 & 1.40 & 1.67 & 1.99 & 2.36 & 1.16 & 1.39 & 1.65 & 1.96 & 1.35 & 1.61 & 1.91 & 2.27 & 1.24 & 1.48 & 1.76 & 2.09 & 1.50 & 1.78 & 2.12 & 2.52 \\
\hline 2026 & 1.42 & 1.71 & 2.05 & 2.46 & 1.19 & 1.43 & 1.72 & 2.07 & 1.39 & 1.67 & 2.01 & 2.41 & 1.27 & 1.53 & 1.83 & 2.20 & 1.54 & 1.85 & 2.23 & 2.67 \\
\hline 2027 & 1.45 & 1.76 & 2.13 & 2.58 & 1.23 & 1.49 & 1.81 & 2.20 & 1.42 & 1.73 & 2.10 & 2.54 & 1.31 & 1.59 & 1.93 & 2.33 & 1.58 & 1.92 & 2.33 & 2.82 \\
\hline 2028 & 1.48 & 1.81 & 2.22 & 2.72 & 1.28 & 1.57 & 1.92 & 2.35 & 1.46 & 1.79 & 2.19 & 2.68 & 1.35 & 1.66 & 2.04 & 2.49 & 1.62 & 1.99 & 2.43 & 2.97 \\
\hline 2029 & 1.51 & 1.88 & 2.32 & 2.86 & 1.31 & 1.63 & 2.01 & 2.48 & 1.50 & 1.86 & 2.31 & 2.85 & 1.40 & 1.73 & 2.15 & 2.65 & 1.66 & 2.06 & 2.54 & 3.14 \\
\hline 2030 & 1.54 & 1.93 & 2.41 & 3.01 & 1.35 & 1.69 & 2.11 & 2.63 & 1.54 & 1.93 & 2.41 & 3.01 & 1.44 & 1.80 & 2.24 & 2.80 & 1.70 & 2.13 & 2.66 & 3.31 \\
\hline 2031 & 1.58 & 1.99 & 2.51 & 3.16 & 1.38 & 1.75 & 2.20 & 2.77 & 1.58 & 1.99 & 2.52 & 3.16 & 1.48 & 1.87 & 2.36 & 2.96 & 1.74 & 2.20 & 2.78 & 3.49 \\
\hline 2032 & 1.61 & 2.06 & 2.62 & 3.33 & 1.41 & 1.80 & 2.30 & 2.92 & 1.61 & 2.06 & 2.62 & 3.33 & 1.53 & 1.95 & 2.48 & 3.15 & 1.79 & 2.28 & 2.90 & 3.69 \\
\hline 2033 & 1.65 & 2.12 & 2.73 & 3.50 & 1.44 & 1.86 & 2.39 & 3.06 & 1.65 & 2.12 & 2.73 & 3.50 & 1.57 & 2.03 & 2.61 & 3.34 & 1.83 & 2.36 & 3.03 & 3.89 \\
\hline 2034 & 1.69 & 2.19 & 2.85 & 3.69 & 1.47 & 1.92 & 2.49 & 3.22 & 1.68 & 2.19 & 2.84 & 3.68 & 1.62 & 2.11 & 2.74 & 3.55 & 1.88 & 2.44 & 3.17 & 4.10 \\
\hline 2035 & 1.72 & 2.27 & 2.97 & 3.88 & 1.50 & 1.98 & 2.59 & 3.39 & 1.72 & 2.26 & 2.96 & 3.87 & 1.67 & 2.20 & 2.88 & 3.77 & 1.92 & 2.53 & 3.31 & 4.33 \\
\hline 2036 & 1.76 & 2.34 & 3.09 & 4.08 & 1.54 & 2.04 & 2.70 & 3.56 & 1.75 & 2.33 & 3.08 & 4.07 & 1.73 & 2.29 & 3.03 & 4.00 & 1.97 & 2.61 & 3.46 & 4.57 \\
\hline 2037 & 1.80 & 2.41 & 3.23 & 4.30 & 1.57 & 2.10 & 2.81 & 3.74 & 1.79 & 2.40 & 3.21 & 4.28 & 1.78 & 2.38 & 3.19 & 4.24 & 2.02 & 2.71 & 3.62 & 4.82 \\
\hline
\end{tabular}


Table S-3. Projected fuel price indices with assumed general price inflation rates of $2 \%, 3 \%, 4 \%$, and $5 \%$, by end-use sector and fuel type.

Census Region 3 (Alabama, Arkansas, Delaware, District of Columbia, Florida, Georgia, Kentucky, Louisiana,

Maryland, Mississippi, North Carolina, Oklahoma, South Carolina, Tennessee, Texas, Virginia, West Virginia)

Projected April 1 Fuel Price Indices (April 1, $2007=1.00$ )

\begin{tabular}{|c|c|c|c|c|c|c|c|c|c|c|c|c|c|c|c|c|}
\hline \multirow[b]{3}{*}{ Year } & \multicolumn{4}{|c|}{ Electricity } & \multicolumn{4}{|c|}{ Distillate oil } & \multicolumn{4}{|c|}{ LPG } & \multicolumn{4}{|c|}{ Natural Gas } \\
\hline & \multicolumn{4}{|c|}{ Inflation Rate } & \multicolumn{4}{|c|}{ Inflation Rate } & \multicolumn{4}{|c|}{ Inflation Rate } & \multicolumn{4}{|c|}{ Inflation Rate } \\
\hline & $2 \%$ & $3 \%$ & $4 \%$ & $5 \%$ & $2 \%$ & $3 \%$ & $4 \%$ & $5 \%$ & $2 \%$ & $3 \%$ & $4 \%$ & $5 \%$ & $2 \%$ & $3 \%$ & $4 \%$ & $5 \%$ \\
\hline 2008 & 1.01 & 1.02 & 1.03 & 1.04 & 1.01 & 1.02 & 1.03 & 1.04 & 1.05 & 1.06 & 1.07 & 1.08 & 0.99 & 1.00 & 1.01 & 1.02 \\
\hline $20 \odot 9$ & 1.02 & 1.04 & 1.06 & $1 . \odot 8$ & $\odot .97$ & 0.99 & 1.01 & 1.03 & 1.05 & 1.07 & 1.09 & 1.12 & 0.98 & $1.0 \odot$ & 1.02 & 1.04 \\
\hline 2010 & 1.03 & 1.06 & 1.09 & 1.13 & 0.93 & 0.96 & 0.98 & 1.01 & 1.06 & 1.09 & 1.12 & 1.15 & 0.97 & $1.0 \odot$ & 1.03 & 1.06 \\
\hline 2011 & 1.04 & 1.08 & 1.12 & 1.16 & $\odot .89$ & 0.93 & 0.97 & $1 . \odot \odot$ & 1.06 & 1.10 & 1.15 & 1.19 & 0.95 & $\odot .99$ & 1.03 & 1.07 \\
\hline 2012 & 1.04 & 1.09 & 1.15 & 1.20 & 0.87 & 0.91 & 0.96 & $1 . \odot \odot$ & 1.07 & 1.12 & 1.18 & 1.24 & 0.95 & $1.0 \odot$ & 1.05 & 1.10 \\
\hline 2013 & 1.05 & 1.12 & 1.18 & 1.25 & 0.84 & 0.89 & 0.94 & $1 . \odot \odot$ & 1.09 & 1.15 & 1.22 & 1.29 & 0.96 & 1.02 & 1.08 & 1.14 \\
\hline 2014 & 1.07 & 1.14 & 1.22 & 1.31 & 0.84 & 0.89 & 0.96 & 1.02 & 1.11 & 1.18 & 1.27 & 1.35 & 0.98 & 1.05 & 1.12 & 1.20 \\
\hline 2015 & 1.08 & 1.17 & 1.27 & 1.37 & 0.85 & 0.92 & 1.00 & 1.07 & 1.13 & 1.22 & 1.32 & 1.42 & 1.00 & 1.08 & 1.16 & 1.26 \\
\hline 2016 & 1.11 & 1.21 & 1.32 & 1.44 & 0.87 & 0.95 & 1.04 & 1.13 & 1.15 & 1.26 & 1.37 & 1.49 & 1.02 & 1.12 & 1.22 & 1.33 \\
\hline 2017 & 1.14 & 1.26 & 1.38 & 1.52 & 0.90 & 0.99 & 1.09 & 1.20 & 1.18 & 1.30 & 1.44 & 1.58 & 1.06 & 1.17 & 1.29 & 1.42 \\
\hline 2018 & 1.17 & 1.30 & 1.45 & 1.61 & 0.93 & 1.03 & 1.15 & 1.27 & 1.21 & 1.35 & 1.50 & 1.66 & 1.09 & 1.22 & 1.35 & 1.50 \\
\hline 2019 & 1.19 & 1.34 & 1.51 & 1.69 & 0.96 & 1.08 & 1.21 & 1.36 & 1.23 & 1.39 & 1.56 & 1.75 & 1.12 & 1.26 & 1.41 & 1.58 \\
\hline 2020 & 1.22 & 1.38 & 1.57 & 1.78 & 0.99 & 1.12 & 1.27 & 1.44 & 1.26 & 1.43 & 1.62 & 1.84 & 1.15 & 1.30 & 1.47 & 1.67 \\
\hline 2021 & 1.24 & 1.42 & 1.63 & 1.87 & 1.01 & 1.16 & 1.33 & 1.52 & 1.28 & 1.47 & 1.69 & 1.93 & 1.17 & 1.34 & 1.54 & 1.76 \\
\hline 2022 & 1.27 & 1.47 & 1.70 & 1.96 & 1.04 & 1.21 & 1.40 & 1.61 & 1.31 & 1.52 & 1.76 & 2.03 & 1.20 & 1.39 & 1.61 & 1.86 \\
\hline 2023 & 1.29 & 1.51 & 1.76 & 2.06 & 1.07 & 1.25 & 1.46 & 1.70 & 1.35 & 1.57 & 1.84 & 2.14 & 1.24 & 1.45 & 1.70 & 1.98 \\
\hline 2024 & 1.32 & 1.56 & 1.84 & 2.17 & 1.10 & 1.29 & 1.53 & 1.80 & 1.38 & 1.63 & 1.92 & 2.26 & 1.29 & 1.52 & 1.79 & 2.10 \\
\hline 2025 & 1.35 & 1.61 & 1.92 & 2.28 & 1.12 & 1.34 & 1.60 & 1.89 & 1.41 & 1.68 & 2.00 & 2.38 & 1.32 & 1.57 & 1.87 & 2.22 \\
\hline 2026 & 1.38 & 1.66 & 1.99 & 2.39 & 1.15 & 1.39 & 1.67 & 2.00 & 1.44 & 1.73 & 2.08 & 2.50 & 1.35 & 1.62 & 1.95 & 2.33 \\
\hline 2027 & 1.41 & 1.71 & 2.08 & 2.51 & 1.19 & 1.44 & 1.75 & 2.12 & 1.47 & 1.79 & 2.17 & 2.63 & 1.38 & 1.68 & 2.04 & 2.47 \\
\hline 2028 & 1.44 & 1.76 & 2.16 & 2.64 & 1.22 & 1.50 & 1.84 & 2.24 & 1.51 & 1.85 & 2.27 & 2.77 & 1.43 & 1.75 & 2.14 & 2.62 \\
\hline 2029 & 1.47 & 1.82 & 2.25 & 2.78 & 1.26 & 1.56 & 1.93 & 2.38 & 1.54 & 1.91 & 2.36 & 2.92 & 1.47 & 1.82 & 2.25 & 2.77 \\
\hline 2030 & 1.50 & 1.88 & 2.35 & 2.93 & 1.29 & 1.62 & 2.02 & 2.52 & 1.58 & 1.97 & 2.46 & 3.07 & 1.50 & 1.88 & 2.35 & 2.92 \\
\hline 2031 & 1.53 & 1.94 & 2.44 & 3.08 & 1.32 & 1.67 & 2.10 & 2.65 & 1.61 & 2.03 & 2.56 & 3.22 & 1.54 & 1.95 & 2.45 & 3.09 \\
\hline 2032 & 1.57 & 2.00 & 2.55 & 3.23 & 1.35 & 1.72 & 2.19 & 2.78 & 1.64 & 2.09 & 2.67 & 3.39 & 1.58 & 2.02 & 2.57 & 3.26 \\
\hline 2033 & 1.60 & 2.06 & 2.65 & 3.40 & 1.38 & 1.77 & 2.28 & 2.92 & 1.67 & 2.16 & 2.77 & 3.56 & 1.62 & 2.09 & 2.69 & 3.45 \\
\hline 2034 & 1.63 & 2.13 & 2.76 & 3.57 & 1.41 & 1.83 & 2.37 & 3.07 & 1.71 & 2.22 & 2.89 & 3.74 & 1.67 & 2.17 & 2.81 & 3.64 \\
\hline 2035 & 1.67 & 2.19 & 2.87 & 3.76 & 1.43 & 1.89 & 2.47 & 3.23 & 1.74 & 2.29 & $3.0 \odot$ & 3.93 & 1.71 & 2.25 & 2.95 & 3.85 \\
\hline 2036 & 1.70 & 2.26 & 2.99 & 3.95 & 1.46 & 1.94 & 2.57 & 3.39 & 1.78 & 2.36 & 3.13 & 4.13 & 1.76 & 2.33 & 3.08 & 4.07 \\
\hline 2037 & 1.74 & 2.33 & 3.12 & 4.15 & 1.50 & $2.0 \odot$ & 2.68 & 3.57 & 1.82 & 2.44 & 3.25 & 4.34 & 1.80 & 2.42 & 3.23 & 4.31 \\
\hline
\end{tabular}


Table S-3, continued. Projected fuel price indices with assumed general price inflation rates of $2 \%, 3 \%, 4 \%$, and $5 \%$, by end-use sector and fuel type.

Census Region 3 (Alabama, Arkansas, Delaware, District of Columbia, Florida, Georgia, Kentucky, Louisiana, Maryland, Mississippi, North Carolina, Oklahoma, South Carolina, Tennessee, Texas, Virginia, West Virginia)

Projected April 1 Fuel Price Indices (April 1, $2007=1.00$ )

\begin{tabular}{lrccc}
\multicolumn{4}{c}{ Electricity } \\
\multicolumn{4}{c}{ Inflation Rate } \\
Year & $2 \%$ & $3 \%$ & $4 \%$ & $5 \%$ \\
2008 & 1.02 & 1.03 & 1.04 & 1.05 \\
2009 & 1.03 & 1.05 & 1.07 & 1.09 \\
2010 & 1.04 & 1.07 & 1.10 & 1.14 \\
2011 & 1.04 & 1.08 & 1.12 & 1.17 \\
2012 & 1.05 & 1.10 & 1.15 & 1.21 \\
2013 & 1.06 & 1.12 & 1.19 & 1.26 \\
2014 & 1.07 & 1.15 & 1.23 & 1.31 \\
2015 & 1.09 & 1.18 & 1.27 & 1.38 \\
2016 & 1.12 & 1.22 & 1.33 & 1.45 \\
2017 & 1.15 & 1.27 & 1.40 & 1.54 \\
2018 & 1.19 & 1.33 & 1.47 & 1.64 \\
2019 & 1.22 & 1.37 & 1.54 & 1.72 \\
2020 & 1.24 & 1.41 & 1.60 & 1.81 \\
2021 & 1.27 & 1.46 & 1.67 & 1.91 \\
2022 & 1.30 & 1.50 & 1.73 & 2.00 \\
2023 & 1.32 & 1.55 & 1.81 & 2.10 \\
2024 & 1.36 & 1.60 & 1.89 & 2.22 \\
2025 & 1.39 & 1.65 & 1.97 & 2.34 \\
2026 & 1.41 & 1.70 & 2.04 & 2.45 \\
2027 & 1.44 & 1.75 & 2.13 & 2.57 \\
2028 & 1.47 & 1.80 & 2.21 & 2.70 \\
2029 & 1.50 & 1.86 & 2.30 & 2.84 \\
2030 & 1.53 & 1.92 & 2.40 & 2.99 \\
2031 & 1.57 & 1.98 & 2.50 & 3.14 \\
2032 & 1.60 & 2.04 & 2.60 & 3.30 \\
2033 & 1.63 & 2.11 & 2.71 & 3.47 \\
2034 & 1.67 & 2.17 & 2.82 & 3.65 \\
2035 & 1.70 & 2.24 & 2.93 & 3.83 \\
2036 & 1.74 & 2.31 & 3.05 & 4.03 \\
2037 & 1.78 & 2.38 & 3.18 & 4.24
\end{tabular}

\begin{tabular}{rrrr}
\multicolumn{4}{c}{ Distillate 0 ill } \\
\hdashline. .4 & Inflation Rate \\
$2 \%$ & $3 \%$ & $4 \%$ & $5 \%$ \\
0.99 & 1.00 & 1.01 & 1.02 \\
0.94 & 0.96 & 0.98 & 1.00 \\
0.90 & 0.93 & 0.96 & 0.99 \\
0.87 & 0.90 & 0.94 & 0.97 \\
0.83 & 0.87 & 0.92 & 0.96 \\
0.81 & 0.86 & 0.91 & 0.96 \\
0.81 & 0.86 & 0.92 & 0.99 \\
0.82 & 0.89 & 0.96 & 1.04 \\
0.85 & 0.93 & 1.01 & 1.10 \\
0.87 & 0.96 & 1.06 & 1.16 \\
0.90 & 1.00 & 1.11 & 1.23 \\
0.93 & 1.05 & 1.18 & 1.32 \\
0.96 & 1.09 & 1.24 & 1.40 \\
0.99 & 1.13 & 1.30 & 1.49 \\
1.03 & 1.19 & 1.37 & 1.59 \\
1.04 & 1.22 & 1.42 & 1.66 \\
1.07 & 1.26 & 1.49 & 1.75 \\
1.11 & 1.32 & 1.57 & 1.87 \\
1.14 & 1.37 & 1.64 & 1.97 \\
1.17 & 1.43 & 1.73 & 2.09 \\
1.22 & 1.49 & 1.83 & 2.24 \\
1.25 & 1.55 & 1.92 & 2.37 \\
1.29 & 1.61 & 2.02 & 2.51 \\
1.32 & 1.67 & 2.10 & 2.65 \\
1.35 & 1.72 & 2.19 & 2.78 \\
1.38 & 1.77 & 2.28 & 2.92 \\
1.41 & 1.83 & 2.37 & 3.07 \\
1.43 & 1.89 & 2.47 & 3.23 \\
1.47 & 1.95 & 2.57 & 3.40 \\
1.50 & 2.01 & 2.68 & 3.57
\end{tabular}

\begin{tabular}{|c|c|c|c|}
\hline \multicolumn{4}{|c|}{ Residual Oil } \\
\hline \multicolumn{4}{|c|}{ Inflation Rate } \\
\hline $2 \%$ & $3 \%$ & $4 \%$ & $5 \%$ \\
\hline 1.04 & 1.05 & 1.06 & 1.07 \\
\hline 1.01 & 1.03 & 1.05 & 1.07 \\
\hline 0.96 & 0.99 & 1.02 & 1.05 \\
\hline 0.93 & 0.97 & 1.01 & 1.05 \\
\hline 0.91 & 0.95 & 1.00 & 1.05 \\
\hline 0.89 & 0.94 & 1.00 & 1.06 \\
\hline 0.88 & 0.94 & 1.01 & 1.08 \\
\hline 0.90 & 0.97 & 1.05 & 1.13 \\
\hline 0.92 & 1.00 & 1.09 & 1.19 \\
\hline 0.95 & 1.05 & 1.15 & 1.27 \\
\hline 0.98 & 1.09 & 1.21 & 1.34 \\
\hline 1.02 & 1.14 & 1.28 & 1.44 \\
\hline 1.05 & 1.20 & 1.36 & 1.54 \\
\hline 1.10 & 1.26 & 1.44 & 1.64 \\
\hline 1.14 & 1.32 & 1.52 & 1.76 \\
\hline 1.15 & 1.35 & 1.57 & 1.83 \\
\hline 1.19 & 1.40 & 1.66 & 1.95 \\
\hline 1.22 & 1.46 & 1.74 & 2.06 \\
\hline 1.26 & 1.52 & 1.82 & 2.19 \\
\hline 1.30 & 1.58 & 1.91 & 2.31 \\
\hline 1.33 & 1.63 & 2.00 & 2.45 \\
\hline 1.38 & 1.71 & 2.11 & 2.60 \\
\hline 1.42 & 1.77 & 2.21 & 2.76 \\
\hline 1.45 & 1.83 & 2.31 & 2.90 \\
\hline 1.48 & 1.89 & 2.40 & 3.05 \\
\hline 1.51 & 1.95 & 2.50 & 3.21 \\
\hline 1.54 & 2.01 & 2.61 & 3.38 \\
\hline 1.58 & 2.07 & 2.72 & 3.55 \\
\hline 1.61 & 2.14 & 2.83 & 3.74 \\
\hline 165 & 2.21 & 2.95 & 3.93 \\
\hline
\end{tabular}

\begin{tabular}{rrrr}
\multicolumn{4}{c}{ Natural Gas } \\
\multicolumn{4}{c}{ Inflation Rate } \\
$2 \%$ & $3 \%$ & $4 \%$ & $5 \%$ \\
0.99 & 1.00 & 1.01 & 1.02 \\
0.97 & 0.98 & 1.00 & 1.02 \\
0.95 & 0.98 & 1.01 & 1.03 \\
0.92 & 0.96 & 1.00 & 1.03 \\
0.91 & 0.96 & 1.01 & 1.05 \\
0.91 & 0.97 & 1.03 & 1.09 \\
0.93 & 1.00 & 1.06 & 1.14 \\
0.94 & 1.02 & 1.10 & 1.19 \\
0.96 & 1.05 & 1.15 & 1.25 \\
1.00 & 1.11 & 1.22 & 1.34 \\
1.03 & 1.15 & 1.27 & 1.42 \\
1.05 & 1.18 & 1.33 & 1.49 \\
1.08 & 1.22 & 1.38 & 1.57 \\
1.09 & 1.25 & 1.44 & 1.64 \\
1.13 & 1.30 & 1.51 & 1.74 \\
1.16 & 1.36 & 1.58 & 1.85 \\
1.20 & 1.42 & 1.67 & 1.97 \\
1.23 & 1.46 & 1.74 & 2.07 \\
1.25 & 1.51 & 1.81 & 2.17 \\
1.29 & 1.56 & 1.89 & 2.29 \\
1.33 & 1.63 & 1.99 & 2.44 \\
1.36 & 1.69 & 2.09 & 2.58 \\
1.39 & 1.74 & 2.18 & 2.71 \\
1.43 & 1.81 & 2.28 & 2.87 \\
1.47 & 1.88 & 2.39 & 3.04 \\
1.51 & 1.95 & 2.51 & 3.21 \\
1.56 & 2.03 & 2.63 & 3.40 \\
1.60 & 2.10 & 2.76 & 3.60 \\
1.65 & 2.19 & 2.89 & 3.82 \\
1.70 & 2.27 & 3.04 & 4.04
\end{tabular}

Coal Inflation Rate $2 \% 3 \% \quad 4 \% \quad 5 \%$ $\begin{array}{llll}1.02 & 1.03 & 1.04 & 1.05\end{array}$ $\begin{array}{llll}1.04 & 1.06 & 1.08 & 1.10\end{array}$ $\begin{array}{llll}1.05 & 1.08 & 1.11 & 1.14\end{array}$ $\begin{array}{llll}1.05 & 1.09 & 1.14 & 1.18\end{array}$ $1.05 \quad 1.10 \quad 1.15 \quad 1.21$ $\begin{array}{llll}1.05 & 1.10 & 1.15 & 1.21\end{array}$ $\begin{array}{llll}1.05 & 1.11 & 1.18 & 1.25 \\ 1.07 & 1.15 & 1.23 & 1.31\end{array}$ $\begin{array}{llll}1.0 & 1.15 & 1.23 & 1.31 \\ 1.10 & 1.19 & 1.28 & 1.38\end{array}$ $\begin{array}{llll}1.10 & 1.19 & 1.28 & 1.38 \\ 1.12 & 1.22 & 1.33 & 1.45\end{array}$ $\begin{array}{llll}1.14 & 1.25 & 1.38 & 1.52\end{array}$ $\begin{array}{llll}1.14 & 1.27 & 1.41 & 1.57\end{array}$ $\begin{array}{llll}1.15 & 1.29 & 1.45 & 1.62\end{array}$ $\begin{array}{lllll}1.16 & 1.31 & 1.49 & 1.69\end{array}$ $\begin{array}{llll}1.16 & 1.31 & 1.49 & 1.69\end{array}$ $1.18 \quad 1.35 \quad 1.55 \quad 1.77$ $\begin{array}{llll}1.21 & 1.40 & 1.62 & 1.87 \\ 1.24 & 1.45 & 1.69 & 1.97\end{array}$ $\begin{array}{llll}1.24 & 1.45 & 1.69 & 1.97 \\ 1.26 & 1.49 & 1.76 & 2.07\end{array}$ $\begin{array}{llll}1.29 & 1.54 & 1.83 & 2.17\end{array}$ $\begin{array}{llll}1.32 & 1.58 & 1.90 & 2.28\end{array}$ $\begin{array}{llll}1.34 & 1.63 & 1.98 & 2.40\end{array}$ $\begin{array}{llll}1.38 & 1.69 & 2.07 & 2.53\end{array}$ $\begin{array}{llll}1.41 & 1.75 & 2.16 & 2.67\end{array}$ $\begin{array}{lll}1.41 & 1.75 & 2.16\end{array}$ $\begin{array}{llll}1.44 & 1.81 & 2.26 & 2.81 \\ 1.48 & 1.87 & 2.36 & 2.97\end{array}$ $\begin{array}{llll}1.48 & 1.87 & 2.36 & 2.97 \\ 1.51 & 1.93 & 2.46 & 3.13\end{array}$ $\begin{array}{llll}1.51 & 1.93 & 2.46 & 3.13 \\ 1.55 & 2.00 & 2.57 & 3.30\end{array}$ $\begin{array}{llll}1.59 & 2.07 & 2.69 & 3.48\end{array}$ $\begin{array}{llll}1.63 & 2.14 & 2.81 & 3.67\end{array}$ $\begin{array}{llll}1.67 & 2.21 & 2.93 & 3.87\end{array}$ $\begin{array}{llll}1.71 & 2.29 & 3.06 & 4.08\end{array}$ 
Table S-3, continued. Projected fuel price indices with assumed general price inflation rates of $2 \%, 3 \%, 4 \%$, and $5 \%$, by end-use sector and fuel type.

Census Region 3 (Alabama, Arkansas, Delaware, District of Columbia, Florida, Georgia, Kentucky, Louisiana, Maryland, Mississippi, North Carolina, Oklahoma, South Carolina, Tennessee, Texas, Virginia, West Virginia)

Projected April 1 Fuel Price Indices (April 1, $2007=1.00$ )

\begin{tabular}{|c|c|c|c|c|}
\hline \multirow[b]{3}{*}{ Year } & \multicolumn{4}{|c|}{ Electricity } \\
\hline & \multicolumn{4}{|c|}{ Inflation Rate } \\
\hline & $2 \%$ & $3 \%$ & $4 \%$ & $5 \%$ \\
\hline 2008 & 1.03 & 1.04 & 1.05 & 1.06 \\
\hline 2009 & 1.04 & 1.06 & 1.08 & 1.10 \\
\hline 2010 & 1.04 & 1.07 & 1.10 & 1.13 \\
\hline 2011 & 1.02 & 1.06 & 1.11 & 1.15 \\
\hline 2012 & 1.02 & 1.07 & 1.12 & 1.18 \\
\hline 2013 & 1.02 & 1.09 & 1.15 & 1.22 \\
\hline 2014 & 1.04 & 1.11 & 1.19 & 1.27 \\
\hline 2015 & 1.05 & 1.14 & 1.23 & 1.33 \\
\hline 2016 & 1.08 & 1.18 & 1.29 & 1.40 \\
\hline 2017 & 1.12 & 1.23 & 1.36 & 1.50 \\
\hline 2018 & 1.16 & 1.29 & 1.43 & 1.59 \\
\hline 2019 & 1.18 & 1.33 & 1.49 & 1.68 \\
\hline 2020 & 1.21 & 1.37 & 1.56 & 1.76 \\
\hline 2021 & 1.24 & 1.42 & 1.62 & 1.86 \\
\hline 2022 & 1.26 & 1.46 & $\begin{array}{l}1.69 \\
1.69\end{array}$ & 1.95 \\
\hline 2023 & 1.29 & 1.51 & 1.76 & 2.05 \\
\hline 2024 & 1.32 & 1.56 & 1.84 & 2.16 \\
\hline 2025 & 1.35 & 1.61 & 1.92 & 2.28 \\
\hline 2026 & 1.38 & 1.66 & 1.99 & 2.39 \\
\hline 2027 & 1.40 & 1.71 & 2.07 & 2.51 \\
\hline 2028 & 1.43 & 1.76 & 2.15 & 2.63 \\
\hline 2029 & 1.46 & 1.82 & 2.25 & 2.77 \\
\hline 2030 & 1.50 & 1.87 & 2.34 & 2.92 \\
\hline 2031 & 1.53 & 1.93 & 2.43 & 3.06 \\
\hline 2032 & 1.56 & 1.99 & 2.54 & 3.22 \\
\hline 2033 & 1.59 & 2.05 & 2.64 & 3.39 \\
\hline 2034 & 1.63 & 2.12 & 2.75 & 3.56 \\
\hline 2035 & 1.66 & 2.18 & 2.86 & 3.74 \\
\hline 2036 & 1.70 & 2.25 & 2.98 & 3.94 \\
\hline 2037 & 1.73 & 2.32 & 3.11 & 4.14 \\
\hline
\end{tabular}

\begin{tabular}{cccc}
\multicolumn{4}{c}{ Distillate 0il } \\
\multicolumn{4}{c}{ Inflation Rate } \\
$2 \%$ & $3 \%$ & $4 \%$ & $5 \%$ \\
0.97 & 0.98 & 0.99 & 1.00 \\
0.93 & 0.95 & 0.96 & 0.98 \\
0.90 & 0.93 & 0.96 & 0.98 \\
0.89 & 0.92 & 0.96 & 1.00 \\
0.87 & 0.91 & 0.95 & 1.00 \\
0.85 & 0.91 & 0.96 & 1.02 \\
0.85 & 0.92 & 0.98 & 1.05 \\
0.87 & 0.94 & 1.02 & 1.10 \\
0.90 & 0.98 & 1.07 & 1.17 \\
0.92 & 1.02 & 1.12 & 1.23 \\
0.95 & 1.06 & 1.17 & 1.30 \\
0.99 & 1.11 & 1.25 & 1.40 \\
1.02 & 1.16 & 1.31 & 1.48 \\
1.05 & 1.20 & 1.37 & 1.57 \\
1.09 & 1.26 & 1.46 & 1.68 \\
1.10 & 1.28 & 1.50 & 1.75 \\
1.13 & 1.33 & 1.57 & 1.84 \\
1.17 & 1.40 & 1.66 & 1.97 \\
1.20 & 1.44 & 1.73 & 2.08 \\
1.24 & 1.50 & 1.83 & 2.21 \\
1.29 & 1.58 & 1.94 & 2.37 \\
1.32 & 1.64 & 2.03 & 2.51 \\
1.36 & 1.71 & 2.13 & 2.66 \\
1.40 & 1.77 & 2.23 & 2.80 \\
1.43 & 1.82 & 2.32 & 2.94 \\
1.46 & 1.88 & 2.41 & 3.09 \\
1.49 & 1.93 & 2.51 & 3.25 \\
1.52 & 2.00 & 2.62 & 3.42 \\
1.55 & 2.06 & 2.72 & 3.59 \\
1.58 & 2.12 & 2.83 & 3.78
\end{tabular}

\begin{tabular}{rrrr}
\multicolumn{4}{c}{ Residual 0il } \\
\hline \multicolumn{4}{c}{ Inflation Rate } \\
$2 \%$ & $3 \%$ & $4 \%$ & $5 \%$ \\
1.06 & 1.07 & 1.09 & 1.10 \\
1.04 & 1.06 & 1.08 & 1.10 \\
1.00 & 1.02 & 1.06 & 1.09 \\
0.97 & 1.01 & 1.05 & 1.09 \\
0.95 & 0.99 & 1.04 & 1.09 \\
0.93 & 0.99 & 1.05 & 1.11 \\
0.93 & 0.99 & 1.06 & 1.14 \\
0.95 & 1.02 & 1.11 & 1.19 \\
0.97 & 1.06 & 1.15 & 1.25 \\
1.00 & 1.10 & 1.21 & 1.33 \\
1.03 & 1.14 & 1.27 & 1.41 \\
1.07 & 1.20 & 1.35 & 1.51 \\
1.10 & 1.25 & 1.42 & 1.61 \\
1.14 & 1.31 & 1.50 & 1.72 \\
1.18 & 1.37 & 1.58 & 1.83 \\
1.20 & 1.40 & 1.64 & 1.91 \\
1.24 & 1.46 & 1.72 & 2.03 \\
1.27 & 1.52 & 1.80 & 2.14 \\
1.31 & 1.58 & 1.89 & 2.27 \\
1.34 & 1.63 & 1.98 & 2.40 \\
1.38 & 1.69 & 2.07 & 2.54 \\
1.42 & 1.76 & 2.18 & 2.69 \\
1.46 & 1.83 & 2.29 & 2.85 \\
1.50 & 1.89 & 2.38 & 3.00 \\
1.53 & 1.95 & 2.48 & 3.16 \\
1.56 & 2.01 & 2.59 & 3.32 \\
1.60 & 2.08 & 2.70 & 3.49 \\
1.63 & 2.14 & 2.81 & 3.67 \\
1.67 & 2.21 & 2.93 & 3.86 \\
1.70 & 2.28 & 3.05 & 4.06
\end{tabular}

\begin{tabular}{rrrr}
\multicolumn{4}{c}{ Natural Gas } \\
\multicolumn{4}{c}{ Inflation Rate } \\
$2 \%$ & $3 \%$ & $4 \%$ & $5 \%$ \\
1.01 & 1.02 & 1.03 & 1.04 \\
0.95 & 0.97 & 0.99 & 1.01 \\
0.91 & 0.94 & 0.97 & 1.00 \\
0.86 & 0.90 & 0.93 & 0.97 \\
0.84 & 0.89 & 0.93 & 0.98 \\
0.84 & 0.89 & 0.94 & 1.00 \\
0.85 & 0.91 & 0.97 & 1.04 \\
0.86 & 0.93 & 1.01 & 1.09 \\
0.89 & 0.97 & 1.06 & 1.16 \\
0.94 & 1.03 & 1.14 & 1.25 \\
0.97 & 1.08 & 1.20 & 1.33 \\
0.99 & 1.11 & 1.25 & 1.40 \\
1.01 & 1.15 & 1.30 & 1.48 \\
1.03 & 1.18 & 1.36 & 1.55 \\
1.07 & 1.24 & 1.43 & 1.65 \\
1.11 & 1.30 & 1.51 & 1.77 \\
1.16 & 1.37 & 1.61 & 1.90 \\
1.18 & 1.41 & 1.68 & 1.99 \\
1.21 & 1.46 & 1.76 & 2.11 \\
1.25 & 1.52 & 1.85 & 2.24 \\
1.30 & 1.59 & 1.95 & 2.38 \\
1.34 & 1.66 & 2.05 & 2.53 \\
1.37 & 1.72 & 2.14 & 2.67 \\
1.42 & 1.79 & 2.26 & 2.84 \\
1.46 & 1.87 & 2.38 & 3.02 \\
1.51 & 1.95 & 2.50 & 3.21 \\
1.56 & 2.03 & 2.64 & 3.42 \\
1.61 & 2.12 & 2.78 & 3.63 \\
1.67 & 2.21 & 2.93 & 3.86 \\
1.72 & 2.31 & 3.09 & 4.11
\end{tabular}

\begin{tabular}{rrrr}
\multicolumn{4}{c}{ Coal } \\
1 & Inflation Rate \\
$2 \%$ & $3 \%$ & $4 \%$ & $5 \%$ \\
1.02 & 1.03 & 1.04 & 1.05 \\
1.04 & 1.06 & 1.08 & 1.10 \\
1.05 & 1.08 & 1.11 & 1.14 \\
1.06 & 1.10 & 1.15 & 1.19 \\
1.07 & 1.12 & 1.18 & 1.24 \\
1.08 & 1.15 & 1.22 & 1.29 \\
1.10 & 1.18 & 1.26 & 1.35 \\
1.12 & 1.21 & 1.31 & 1.41 \\
1.14 & 1.24 & 1.35 & 1.47 \\
1.16 & 1.28 & 1.41 & 1.55 \\
1.17 & 1.30 & 1.45 & 1.61 \\
1.19 & 1.34 & 1.50 & 1.69 \\
1.21 & 1.38 & 1.56 & 1.77 \\
1.24 & 1.42 & 1.63 & 1.86 \\
1.26 & 1.46 & 1.69 & 1.95 \\
1.29 & 1.51 & 1.77 & 2.06 \\
1.32 & 1.56 & 1.84 & 2.16 \\
1.35 & 1.61 & 1.92 & 2.28 \\
1.38 & 1.67 & 2.00 & 2.40 \\
1.41 & 1.72 & 2.08 & 2.52 \\
1.45 & 1.77 & 2.17 & 2.66 \\
1.48 & 1.84 & 2.27 & 2.80 \\
1.52 & 1.90 & 2.37 & 2.95 \\
1.55 & 1.96 & 2.47 & 3.11 \\
1.59 & 2.03 & 2.58 & 3.28 \\
1.63 & 2.10 & 2.70 & 3.46 \\
1.67 & 2.17 & 2.82 & 3.65 \\
1.71 & 2.24 & 2.94 & 3.85 \\
1.75 & 2.32 & 3.07 & 4.05 \\
1.79 & 2.40 & 3.21 & 4.27
\end{tabular}


Table S-4. Projected fuel price indices with assumed general price inflation rates of $2 \%, 3 \%, 4 \%$, and $5 \%$, by end-use sector and fuel type.

Census Region 4 (Alaska, Arizona, California, Colorado, Hawaii,

Idaho, Montana, Nevada, New Mexico, Oregon, Utah, Washington, Wyoming)

Projected April 1 Fuel Price Indices (April 1, $2007=1.0 \odot)$

\begin{tabular}{|c|c|c|c|c|c|c|c|c|c|c|c|c|c|c|c|c|}
\hline \multicolumn{5}{|c|}{ Electricity } & \multicolumn{4}{|c|}{ Distillate 0il } & \multicolumn{4}{|c|}{ LPG } & \multicolumn{4}{|c|}{ Natural Gas } \\
\hline & \multicolumn{4}{|c|}{ Inflation Rate } & \multicolumn{4}{|c|}{ Inflation Rate } & \multicolumn{4}{|c|}{ Inflation Rate } & \multicolumn{4}{|c|}{ Inflation Rate } \\
\hline Year & $2 \%$ & $3 \%$ & $4 \%$ & $5 \%$ & $2 \%$ & $3 \%$ & $4 \%$ & $5 \%$ & $2 \%$ & $3 \%$ & $4 \%$ & $5 \%$ & $2 \%$ & $3 \%$ & $4 \%$ & $5 \%$ \\
\hline $20 \odot 8$ & 1.02 & 1.03 & 1.04 & 1.05 & 0.98 & $\odot .99$ & $1.0 \odot$ & $1 . \odot \odot$ & 1.05 & 1.06 & 1.07 & 1.08 & $\odot .99$ & 1.00 & 1.01 & 1.02 \\
\hline $20 \odot 9$ & 1.03 & 1.05 & 1.07 & 1.09 & 0.95 & 0.97 & 0.99 & 1.01 & 1.06 & 1.08 & 1.10 & 1.12 & 0.98 & 1.00 & 1.02 & 1.04 \\
\hline 2010 & 1.03 & 1.06 & 1.09 & 1.12 & $\odot .91$ & 0.93 & 0.96 & 0.99 & 1.06 & 1.09 & 1.13 & 1.16 & 0.97 & 1.00 & 1.03 & 1.06 \\
\hline 2011 & 1.03 & 1.07 & 1.11 & 1.15 & 0.87 & 0.91 & 0.94 & 0.98 & 1.07 & 1.11 & 1.16 & 1.20 & 0.97 & 1.00 & 1.04 & 1.08 \\
\hline 2012 & 1.03 & 1.08 & 1.14 & 1.19 & 0.84 & 0.88 & 0.93 & 0.97 & 1.08 & 1.14 & 1.19 & 1.25 & 0.97 & 1.02 & 1.07 & 1.12 \\
\hline 2013 & 1.05 & 1.11 & 1.18 & 1.25 & $\odot .80$ & 0.85 & 0.90 & 0.96 & 1.09 & 1.16 & 1.23 & 1.30 & 0.97 & 1.03 & 1.09 & 1.15 \\
\hline 2014 & 1.06 & 1.14 & 1.22 & 1.30 & 0.81 & 0.87 & 0.93 & 0.99 & 1.11 & 1.19 & 1.28 & 1.37 & 0.99 & 1.06 & 1.13 & 1.21 \\
\hline 2015 & 1.09 & 1.17 & 1.27 & 1.37 & 0.82 & 0.89 & 0.96 & 1.04 & 1.14 & 1.23 & 1.33 & 1.43 & 1.00 & 1.09 & 1.17 & 1.27 \\
\hline 2016 & 1.12 & 1.22 & 1.33 & 1.45 & 0.84 & 0.92 & 1.00 & 1.10 & 1.16 & 1.27 & 1.38 & 1.51 & 1.03 & 1.12 & 1.23 & 1.34 \\
\hline 2017 & 1.15 & 1.27 & 1.39 & 1.53 & 0.87 & 0.96 & 1.06 & 1.16 & 1.19 & 1.31 & 1.45 & 1.59 & 1.07 & 1.18 & 1.30 & 1.43 \\
\hline 2018 & 1.17 & 1.30 & 1.45 & 1.61 & $\odot .9 \odot$ & $1 . \odot \odot$ & 1.11 & 1.24 & 1.21 & 1.34 & 1.49 & 1.66 & 1.08 & 1.20 & 1.33 & 1.48 \\
\hline 2019 & 1.19 & 1.33 & 1.50 & 1.68 & 0.94 & 1.05 & 1.18 & 1.33 & 1.22 & 1.37 & 1.54 & 1.73 & 1.07 & 1.21 & 1.36 & 1.52 \\
\hline 2020 & 1.21 & 1.37 & 1.56 & 1.76 & 0.97 & 1.10 & 1.25 & 1.41 & 1.25 & 1.42 & 1.60 & 1.82 & 1.10 & 1.25 & 1.42 & 1.61 \\
\hline 2021 & 1.24 & 1.42 & 1.63 & 1.86 & 1.00 & 1.14 & 1.31 & 1.50 & 1.28 & 1.46 & 1.67 & 1.91 & 1.13 & 1.30 & 1.49 & 1.70 \\
\hline 2022 & 1.27 & 1.47 & 1.70 & 1.96 & 1.04 & 1.20 & 1.39 & 1.60 & 1.31 & 1.51 & 1.75 & 2.02 & 1.17 & 1.35 & 1.57 & 1.81 \\
\hline 2023 & 1.30 & 1.52 & 1.77 & 2.07 & 1.06 & 1.24 & 1.45 & 1.69 & 1.34 & 1.57 & 1.83 & 2.14 & 1.22 & 1.42 & 1.66 & 1.93 \\
\hline 2024 & 1.33 & 1.57 & 1.85 & 2.18 & 1.09 & 1.28 & 1.51 & 1.78 & 1.38 & 1.63 & 1.92 & 2.25 & 1.26 & 1.48 & 1.75 & 2.05 \\
\hline 2025 & 1.36 & 1.62 & 1.93 & 2.29 & 1.13 & 1.35 & 1.61 & 1.91 & 1.41 & 1.68 & 2.00 & 2.37 & 1.29 & 1.54 & 1.83 & 2.17 \\
\hline 2026 & 1.39 & 1.68 & 2.01 & 2.42 & 1.17 & 1.40 & 1.69 & 2.02 & 1.44 & 1.73 & 2.08 & 2.49 & 1.32 & 1.59 & 1.91 & 2.29 \\
\hline 2027 & 1.42 & 1.73 & 2.10 & 2.54 & 1.20 & 1.46 & 1.77 & 2.15 & 1.47 & 1.79 & 2.17 & 2.63 & 1.36 & 1.65 & 2.00 & 2.42 \\
\hline 2028 & 1.45 & 1.78 & 2.19 & 2.67 & 1.24 & 1.53 & 1.8 & 2.29 & & $1 . \varepsilon$ & 2.27 & 2.77 & 1.40 & 1.72 & 2.11 & 2.58 \\
\hline 2029 & 1.49 & 1.85 & 2.29 & 2.82 & 1.28 & 1.59 & 1.97 & 2.43 & 1. & 1.92 & 2.37 & 2.93 & 1.45 & 1.80 & 2.22 & 2.75 \\
\hline 2030 & 1.53 & 1.91 & 2.39 & 2.98 & 1.32 & 1.65 & 2.06 & 2.57 & 1.58 & 1.98 & 2.48 & 3.09 & 1.50 & 1.87 & 2.34 & 2.91 \\
\hline 2031 & 1.57 & 1.98 & 2.49 & 3.14 & 1.35 & 1.70 & 2.15 & 2.70 & 1.62 & 2.04 & 2.58 & 3.24 & 1.54 & 1.94 & 2.45 & 3.08 \\
\hline 2032 & 1.60 & 2.05 & 2.60 & 3.31 & 1.38 & 1.76 & 2.24 & 2.84 & 1.65 & 2.11 & 2.68 & 3.41 & 1.58 & 2.01 & 2.57 & 3.26 \\
\hline 2033 & 1.64 & 2.11 & 2.72 & 3.49 & 1.40 & 1.81 & 2.33 & 2.98 & 1.69 & 2.17 & 2.79 & 3.58 & 1.62 & 2.09 & 2.69 & 3.45 \\
\hline 2034 & 1.68 & 2.19 & 2.84 & 3.67 & 1.43 & 1.87 & 2.42 & 3.14 & 1.72 & 2.24 & 2.90 & 3.76 & 1.67 & 2.17 & 2.82 & 3.65 \\
\hline 2035 & 1.72 & 2.26 & 2.96 & 3.87 & 1.46 & 1.92 & 2.52 & 3.30 & 1.76 & 2.31 & 3.02 & 3.95 & 1.71 & 2.25 & 2.95 & 3.86 \\
\hline 2036 & 1.76 & 2.34 & 3.09 & 4.08 & 1.50 & 1.98 & 2.63 & 3.47 & 1.79 & 2.38 & 3.15 & 4.15 & 1.76 & 2.34 & 3.09 & 4.08 \\
\hline 2037 & 1.80 & 2.42 & 3.23 & 4.30 & 1.53 & 2.04 & 2.73 & 3.64 & 1.83 & 2.45 & 3.27 & 4.36 & 1.81 & 2.43 & 3.24 & 4.32 \\
\hline
\end{tabular}


Table S-4, continued. Projected fuel price indices with assumed general price inflation rates of $2 \%, 3 \%, 4 \%$, and $5 \%$, by end-use sector and fuel type.

Census Region 4 (Alaska, Arizona, California, Colorado, Hawaii,

Idaho, Montana, Nevada, New Mexico, Oregon, Utah, Washington, Wyoming)

Projected April 1 Fuel Price Indices (April 1, $2007=1.00$ )

\begin{tabular}{|c|c|c|c|c|}
\hline \multirow[b]{3}{*}{ Year } & \multicolumn{4}{|c|}{ Electricity } \\
\hline & \multicolumn{4}{|c|}{ Inflation Rate } \\
\hline & $2 \%$ & $3 \%$ & $4 \%$ & $5 \%$ \\
\hline 2008 & 1.01 & 1.02 & 1.03 & 1.04 \\
\hline 2009 & 1.02 & 1.04 & 1.06 & 1.08 \\
\hline 2010 & 1.01 & 1.04 & 1.07 & 1.11 \\
\hline 2011 & 1.00 & 1.04 & 1.08 & 1.13 \\
\hline 2012 & 1.00 & 1.05 & 1.10 & 1.16 \\
\hline 2013 & 1.01 & 1.07 & 1.14 & 1.21 \\
\hline 2014 & 1.03 & 1.10 & 1.18 & 1.26 \\
\hline 2015 & 1.05 & 1.13 & 1.23 & 1.32 \\
\hline 2016 & 1.08 & 1.18 & 1.28 & 1.40 \\
\hline 2017 & 1.11 & 1.22 & 1.35 & 1.48 \\
\hline 2018 & 1.13 & 1.26 & 1.40 & 1.56 \\
\hline 2019 & 1.14 & 1.28 & 1.44 & 1.62 \\
\hline 2020 & 1.16 & 1.32 & 1.50 & 1.70 \\
\hline 2021 & 1.19 & 1.36 & 1.56 & 1.79 \\
\hline 2022 & 1.22 & 1.41 & 1.63 & 1.88 \\
\hline 2023 & 1.24 & 1.45 & 1.70 & 1.98 \\
\hline 2024 & 1.27 & 1.50 & 1.77 & 2.08 \\
\hline 2025 & 1.30 & 1.55 & 1.84 & 2.19 \\
\hline 2026 & 1.33 & 1.60 & 1.92 & 2.31 \\
\hline 2027 & 1.36 & 1.65 & 2.00 & 2.42 \\
\hline 2028 & 1.39 & 1.70 & 2.08 & 2.55 \\
\hline 2029 & 1.42 & 1.76 & 2.18 & 2.69 \\
\hline 2030 & 1.46 & 1.82 & 2.27 & 2.84 \\
\hline 2031 & 1.49 & 1.88 & 2.37 & 2.98 \\
\hline 2032 & 1.52 & 1.94 & 2.47 & 3.14 \\
\hline 2033 & 1.56 & 2.01 & 2.58 & 3.31 \\
\hline 2034 & 1.59 & 2.07 & 2.69 & 3.48 \\
\hline 2035 & 1.63 & 2.14 & 2.80 & 3.66 \\
\hline 2036 & 1.66 & 2.21 & 2.92 & 3.86 \\
\hline 2037 & 1.70 & 2.28 & 3.05 & 4.06 \\
\hline
\end{tabular}

\begin{tabular}{rrrr}
\multicolumn{4}{c}{ Distillate 0il } \\
\hdashline.-1 Inflation Rate \\
$2 \%$ & $3 \%$ & $4 \%$ & $5 \%$ \\
0.96 & 0.97 & 0.98 & 0.99 \\
0.93 & 0.95 & 0.97 & 0.98 \\
0.90 & 0.93 & 0.95 & 0.98 \\
0.87 & 0.90 & 0.94 & 0.97 \\
0.83 & 0.88 & 0.92 & 0.96 \\
0.82 & 0.87 & 0.92 & 0.98 \\
0.81 & 0.87 & 0.93 & 0.99 \\
0.82 & 0.89 & 0.96 & 1.04 \\
0.85 & 0.93 & 1.01 & 1.10 \\
0.88 & 0.97 & 1.07 & 1.17 \\
0.91 & 1.01 & 1.12 & 1.25 \\
0.95 & 1.07 & 1.20 & 1.34 \\
0.97 & 1.10 & 1.25 & 1.41 \\
0.99 & 1.14 & 1.30 & 1.49 \\
1.03 & 1.20 & 1.38 & 1.60 \\
1.05 & 1.23 & 1.44 & 1.68 \\
1.08 & 1.28 & 1.50 & 1.77 \\
1.12 & 1.34 & 1.60 & 1.90 \\
1.15 & 1.39 & 1.67 & 2.00 \\
1.19 & 1.44 & 1.75 & 2.12 \\
1.24 & 1.52 & 1.86 & 2.27 \\
1.27 & 1.57 & 1.94 & 2.40 \\
1.31 & 1.63 & 2.04 & 2.54 \\
1.34 & 1.69 & 2.13 & 2.68 \\
1.37 & 1.74 & 2.22 & 2.82 \\
1.40 & 1.80 & 2.31 & 2.97 \\
1.42 & 1.85 & 2.41 & 3.12 \\
1.45 & 1.91 & 2.50 & 3.27 \\
1.49 & 1.97 & 2.61 & 3.44 \\
1.52 & 2.03 & 2.72 & 3.62
\end{tabular}

- - COMMERCIAL

Residual oil

Natural Gas

Coal

Inflation Rate

$2 \% \quad 3 \% \quad 4 \% \quad 5 \%$

1.051 .07

$\begin{array}{llll}0.92 & 0.94 & 0.97 & 1.00\end{array}$

$\begin{array}{llll}0.85 & 0.89 & 0.92 & 0.96 \\ 0.82 & 0.86 & 0.90 & 0.95\end{array}$

$\begin{array}{llll}0.82 & 0.86 & 0.90 & 0.95 \\ 0.79 & 0.84 & 0.89 & 0.94\end{array}$

$\begin{array}{llll}0.77 & 0.82 & 0.88 & 0.94\end{array}$

$0.78 \quad 0.85 \quad 0.91 \quad 0.99$

$0.80 \quad 0.87 \quad 0.95 \quad 1.04$

$0.83 \quad 0.92 \quad 1.01 \quad 1.11$

$0.86 \quad 0.96 \quad 1.07 \quad 1.18$

$0.90 \quad 1.02 \quad 1.14 \quad 1.28$

$\begin{array}{llll}0.94 & 1.07 & 1.21 & 1.38\end{array}$

$\begin{array}{llll}0.94 & 1.07 & 1.21 & 1.38 \\ 0.99 & 1.13 & 1.30 & 1.48\end{array}$

$\begin{array}{llll}1.02 & 1.19 & 1.37 & 1.58\end{array}$

$1.03 \quad 1.21 \quad 1.41 \quad 1.64$

$1.07 \quad 1.27 \quad 1.49 \quad 1.76$

$\begin{array}{llll}1.11 & 1.32 & 1.57 & 1.86\end{array}$

$\begin{array}{llll}1.15 & 1.38 & 1.66 & 1.99\end{array}$

$\begin{array}{llll}1.18 & 1.44 & 1.74 & 2.11\end{array}$

$\begin{array}{llll}1.21 & 1.49 & 1.82 & 2.23\end{array}$

$\begin{array}{llll}1.26 & 1.56 & 1.93 & 2.38\end{array}$

$\begin{array}{llll}1.30 & 1.62 & 2.03 & 2.53\end{array}$

$\begin{array}{llll}1.33 & 1.68 & 2.12 & 2.66\end{array}$

$\begin{array}{llll}1.36 & 1.73 & 2.21 & 2.81\end{array}$

$\begin{array}{llll}1.39 & 1.79 & 2.30 & 2.95\end{array}$

$\begin{array}{llll}1.42 & 1.85 & 2.40 & 3.10\end{array}$

$\begin{array}{llll}1.45 & 1.91 & 2.50 & 3.27\end{array}$

$\begin{array}{llll}1.48 & 1.97 & 2.61 & 3.44\end{array}$

$\begin{array}{llll}1.52 & 2.04 & 2.72 & 3.62\end{array}$

\begin{tabular}{cccc}
\multicolumn{4}{c}{ Inflation Rate } \\
0.99 & $3 \%$ & $4 \%$ & $5 \%$ \\
0.99 & 1.00 & 1.01 & 1.02 \\
0.98 & 0.99 & 1.01 & 1.03 \\
0.96 & 0.99 & 1.02 & 1.05 \\
0.95 & 0.99 & 1.02 & 1.06 \\
0.95 & 1.00 & 1.05 & 1.10 \\
0.94 & 1.00 & 1.06 & 1.12 \\
0.95 & 1.02 & 1.09 & 1.17 \\
0.97 & 1.05 & 1.13 & 1.22 \\
0.99 & 1.08 & 1.18 & 1.29 \\
1.03 & 1.13 & 1.25 & 1.37 \\
1.03 & 1.15 & 1.27 & 1.42 \\
1.02 & 1.15 & 1.29 & 1.45 \\
1.05 & 1.19 & 1.35 & 1.53 \\
1.08 & 1.23 & 1.41 & 1.61 \\
1.11 & 1.29 & 1.49 & 1.72 \\
1.15 & 1.35 & 1.57 & 1.83 \\
1.19 & 1.40 & 1.65 & 1.95 \\
1.22 & 1.45 & 1.73 & 2.05 \\
1.25 & 1.50 & 1.80 & 2.16 \\
1.28 & 1.55 & 1.89 & 2.28 \\
1.32 & 1.62 & 1.99 & 2.43 \\
1.37 & 1.69 & 2.09 & 2.59 \\
1.43 & 1.79 & 2.23 & 2.78 \\
1.48 & 1.87 & 2.35 & 2.96 \\
1.52 & 1.94 & 2.47 & 3.13 \\
1.56 & 2.01 & 2.59 & 3.32 \\
1.61 & 2.09 & 2.71 & 3.51 \\
1.65 & 2.17 & 2.85 & 3.72 \\
1.70 & 2.26 & 2.99 & 3.94 \\
1.75 & 2.35 & 3.13 & 4.18
\end{tabular}

Inflation Rate

$2 \% \quad 3 \% \quad 4 \% \quad 5 \%$

$\begin{array}{llll}1.03 & 1.04 & 1.05 & 1.06\end{array}$

$\begin{array}{llll}1.06 & 1.08 & 1.10 & 1.12\end{array}$

$\begin{array}{lllll}1.09 & 1.08 & 1.10 & 1.12\end{array}$

1.09 1.12 $1.15,1.19$

$1.10 \quad 1.15 \quad 1.19 \quad 1.24$

$\begin{array}{llll}1.13 & 1.18 & 1.24 & 1.30\end{array}$

$\begin{array}{llll}1.14 & 1.21 & 1.28 & 1.36\end{array}$

$\begin{array}{llll}1.17 & 1.25 & 1.34 & 1.44\end{array}$

$\begin{array}{llll}1.20 & 1.30 & 1.40 & 1.52\end{array}$

$\begin{array}{llll}1.23 & 1.34 & 1.47 & 1.60\end{array}$

$\begin{array}{llll}1.26 & 1.39 & 1.53 & 1.69\end{array}$

$\begin{array}{llll}1.30 & 1.45 & 1.61 & 1.69\end{array}$

$1.34 \quad 1.51$ 1.61 1.79

$\begin{array}{llll}1.34 & 1.51 & 1.69 & 1.90 \\ 1.38 & 1.57 & 1.78 & 2.01\end{array}$

$\begin{array}{llll}1.38 & 1.57 & 1.78 & 2.01 \\ 1.43 & 1.64 & 1.87 & 2.14\end{array}$

$\begin{array}{llll}1.48 & 1.71 & 1.98 & 2.28\end{array}$

$\begin{array}{llll}1.52 & 1.78 & 2.07 & 2.42\end{array}$

$\begin{array}{llll}1.56 & 1.84 & 2.17 & 2.55\end{array}$

$\begin{array}{llll}1.60 & 1.91 & 2.27 & 2.70\end{array}$

$\begin{array}{llll}1.65 & 1.99 & 2.39 & 2.86\end{array}$

$\begin{array}{llll}1.70 & 2.06 & 2.50 & 3.03\end{array}$

$\begin{array}{llll}1.75 & 2.15 & 2.63 & 3.21\end{array}$

$\begin{array}{llll}1.80 & 2.23 & 2.76 & 3.40\end{array}$

$\begin{array}{llll}1.84 & 2.31 & 2.88 & 3.59\end{array}$

$\begin{array}{llll}1.89 & 2.39 & 3.01 & 3.78\end{array}$

$\begin{array}{llll}1.93 & 2.47 & 3.14 & 3.99\end{array}$

$\begin{array}{llll}1.98 & 2.55 & 3.28 & 4.21\end{array}$

$\begin{array}{llll}2.03 & 2.64 & 3.43 & 4.44\end{array}$

$\begin{array}{llll}2.08 & 2.73 & 3.58 & 4.68\end{array}$

$\begin{array}{llll}2.13 & 2.83 & 3.74 & 4.94\end{array}$

$\begin{array}{llll}2.18 & 2.92 & 3.91 & 5.20\end{array}$ 
Table S-4, continued. Projected fuel price indices with assumed general price inflation rates of $2 \%, 3 \%, 4 \%$, and $5 \%$, by end-use sector and fuel type.

Census Region 4 (Alaska, Arizona, California, Colorado, Hawaii,

Idaho, Montana, Nevada, New Mexico, Oregon, Utah, Washington, Wyoming)

Projected April 1 Fuel Price Indices (April 1, $2007=1.00$ )

\begin{tabular}{|c|c|c|c|c|}
\hline & \multicolumn{4}{|c|}{ Electricity } \\
\hline & \multicolumn{4}{|c|}{ Inflation Rate } \\
\hline Year & $2 \%$ & $3 \%$ & $4 \%$ & $5 \%$ \\
\hline 2008 & 1.03 & 1.04 & 1.05 & 1.06 \\
\hline 2009 & 1.03 & 1.05 & 1.07 & 1.10 \\
\hline 2010 & 1.03 & 1.06 & 1.10 & 1.13 \\
\hline 2011 & 1.02 & 1.06 & 1.11 & 1.15 \\
\hline 2012 & 1.02 & 1.07 & 1.12 & 1.18 \\
\hline 2013 & 1.03 & 1.09 & 1.16 & 1.23 \\
\hline 2014 & 1.04 & 1.12 & 1.20 & 1.28 \\
\hline 2015 & 1.07 & 1.15 & 1.25 & 1.35 \\
\hline 2016 & 1.10 & 1.20 & 1.31 & 1.43 \\
\hline 2017 & 1.13 & 1.25 & 1.38 & 1.51 \\
\hline 2018 & 1.16 & 1.29 & 1.43 & 1.59 \\
\hline 2019 & 1.17 & 1.32 & 1.48 & 1.66 \\
\hline 2020 & 1.19 & 1.35 & 1.53 & 1.74 \\
\hline 2021 & 1.22 & 1.40 & 1.60 & 1.83 \\
\hline 2022 & 1.25 & 1.45 & 1.68 & 1.93 \\
\hline 2023 & 1.28 & 1.49 & 1.74 & 2.03 \\
\hline 2024 & 1.31 & 1.54 & 1.82 & 2.14 \\
\hline 2025 & 1.34 & 1.60 & 1.90 & 2.26 \\
\hline 2026 & 1.37 & 1.65 & 1.98 & 2.38 \\
\hline 2027 & $1.4 \odot$ & 1.70 & 2.07 & 2.50 \\
\hline 2028 & 1.43 & 1.76 & 2.15 & 2.63 \\
\hline 2029 & 1.47 & 1.83 & 2.26 & 2.79 \\
\hline 2030 & 1.51 & 1.89 & 2.36 & 2.94 \\
\hline 2031 & 1.55 & 1.96 & 2.47 & 3.10 \\
\hline 2032 & 1.58 & 2.02 & 2.57 & 3.27 \\
\hline 2033 & 1.62 & 2.09 & 2.69 & 3.45 \\
\hline 2034 & 1.66 & 2.16 & 2.81 & 3.63 \\
\hline 2035 & 1.70 & 2.24 & 2.93 & 3.83 \\
\hline 2036 & 1.74 & 2.31 & 3.06 & 4.04 \\
\hline 2037 & 1.78 & 2.39 & 3.20 & 4.26 \\
\hline
\end{tabular}

\begin{tabular}{lccc}
\multicolumn{4}{c}{ Distillate 0 il } \\
\hline \multicolumn{4}{c}{ Inflation Rate } \\
$2 \%$ & $3 \%$ & $4 \%$ & $5 \%$ \\
0.95 & 0.96 & 0.97 & 0.98 \\
0.92 & 0.93 & 0.95 & 0.97 \\
0.90 & 0.93 & 0.95 & 0.98 \\
0.88 & 0.92 & 0.95 & 0.99 \\
0.85 & 0.90 & 0.94 & 0.99 \\
0.86 & 0.91 & 0.97 & 1.02 \\
0.84 & 0.90 & 0.97 & 1.03 \\
0.85 & 0.92 & 1.00 & 1.08 \\
0.89 & 0.97 & 1.06 & 1.15 \\
0.92 & 1.01 & 1.11 & 1.22 \\
0.94 & 1.05 & 1.17 & 1.29 \\
0.98 & 1.11 & 1.24 & 1.39 \\
1.00 & 1.13 & 1.28 & 1.46 \\
1.02 & 1.17 & 1.33 & 1.53 \\
1.06 & 1.23 & 1.42 & 1.64 \\
1.08 & 1.26 & 1.47 & 1.71 \\
1.11 & 1.31 & 1.54 & 1.81 \\
1.15 & 1.37 & 1.63 & 1.94 \\
1.18 & 1.42 & 1.70 & 2.04 \\
1.21 & 1.47 & 1.78 & 2.16 \\
1.26 & 1.55 & 1.90 & 2.32 \\
1.29 & 1.59 & 1.97 & 2.43 \\
1.33 & 1.66 & 2.08 & 2.59 \\
1.36 & 1.72 & 2.17 & 2.73 \\
1.39 & 1.78 & 2.26 & 2.87 \\
1.42 & 1.83 & 2.36 & 3.02 \\
1.45 & 1.89 & 2.45 & 3.17 \\
1.48 & 1.95 & 2.55 & 3.34 \\
1.51 & 2.01 & 2.66 & 3.51 \\
1.54 & 2.07 & 2.77 & 3.69
\end{tabular}

\begin{tabular}{rrrr}
\multicolumn{4}{c}{ Residual 0il } \\
\multicolumn{4}{c}{ Inflation Rate } \\
$2 \%$ & $3 \%$ & $4 \%$ & $5 \%$ \\
1.05 & 1.06 & 1.07 & 1.08 \\
1.04 & 1.06 & 1.08 & 1.10 \\
0.98 & 1.01 & 1.04 & 1.07 \\
0.94 & 0.98 & 1.02 & 1.06 \\
0.92 & 0.96 & 1.01 & 1.06 \\
0.90 & 0.95 & 1.01 & 1.07 \\
0.89 & 0.96 & 1.02 & 1.09 \\
0.91 & 0.99 & 1.07 & 1.15 \\
0.93 & 1.01 & 1.11 & 1.21 \\
0.96 & 1.06 & 1.17 & 1.28 \\
0.99 & 1.10 & 1.22 & 1.36 \\
1.02 & 1.15 & 1.29 & 1.45 \\
1.06 & 1.20 & 1.36 & 1.54 \\
1.10 & 1.26 & 1.44 & 1.65 \\
1.13 & 1.31 & 1.52 & 1.75 \\
1.16 & 1.36 & 1.58 & 1.85 \\
1.20 & 1.42 & 1.67 & 1.97 \\
1.23 & 1.47 & 1.75 & 2.07 \\
1.28 & 1.54 & 1.85 & 2.21 \\
1.32 & 1.61 & 1.95 & 2.37 \\
1.35 & 1.65 & 2.03 & 2.48 \\
1.41 & 1.74 & 2.15 & 2.66 \\
1.43 & 1.79 & 2.24 & 2.79 \\
1.46 & 1.84 & 2.32 & 2.92 \\
1.49 & 1.90 & 2.42 & 3.08 \\
1.52 & 1.96 & 2.52 & 3.23 \\
1.56 & 2.02 & 2.63 & 3.40 \\
1.59 & 2.09 & 2.74 & 3.58 \\
1.62 & 2.16 & 2.85 & 3.76 \\
1.66 & 2.22 & 2.97 & 3.96
\end{tabular}

\begin{tabular}{cccc}
\multicolumn{4}{c}{ Natural Gas } \\
\hline \multicolumn{4}{c}{ Inflation Rate } \\
$2 \%$ & $3 \%$ & $4 \%$ & $5 \%$ \\
1.02 & 1.03 & 1.04 & 1.05 \\
0.99 & 1.01 & 1.03 & 1.05 \\
0.96 & 0.99 & 1.02 & 1.05 \\
0.94 & 0.97 & 1.01 & 1.05 \\
0.93 & 0.98 & 1.03 & 1.08 \\
0.91 & 0.97 & 1.03 & 1.09 \\
0.92 & 0.99 & 1.06 & 1.13 \\
0.94 & 1.02 & 1.10 & 1.19 \\
0.96 & 1.05 & 1.15 & 1.25 \\
1.01 & 1.11 & 1.22 & 1.35 \\
1.00 & 1.12 & 1.24 & 1.38 \\
0.99 & 1.11 & 1.25 & 1.40 \\
1.02 & 1.16 & 1.31 & 1.49 \\
1.05 & 1.20 & 1.38 & 1.58 \\
1.09 & 1.26 & 1.46 & 1.68 \\
1.14 & 1.33 & 1.56 & 1.81 \\
1.18 & 1.39 & 1.64 & 1.93 \\
1.21 & 1.45 & 1.72 & 2.05 \\
1.24 & 1.50 & 1.80 & 2.16 \\
1.28 & 1.56 & 1.89 & 2.29 \\
1.33 & 1.64 & 2.00 & 2.45 \\
1.39 & 1.72 & 2.13 & 2.62 \\
1.43 & 1.79 & 2.24 & 2.79 \\
1.48 & 1.87 & 2.36 & 2.96 \\
1.53 & 1.95 & 2.48 & 3.15 \\
1.57 & 2.03 & 2.61 & 3.34 \\
1.62 & 2.11 & 2.74 & 3.55 \\
1.68 & 2.20 & 2.89 & 3.78 \\
1.73 & 2.30 & 3.04 & 4.01 \\
1.79 & 2.39 & 3.20 & 4.26
\end{tabular}

Coal

Inflation Rate

$2 \% \quad 3 \% \quad 4 \% \quad 5 \%$

$\begin{array}{llll}1.03 & 1.04 & 1.05 & 1.06\end{array}$

$\begin{array}{llll}1.04 & 1.06 & 1.08 & 1.10\end{array}$

$\begin{array}{llll}1.07 & 1.10 & 1.14 & 1.17\end{array}$

$\begin{array}{llll}1.09 & 1.13 & 1.18 & 1.22\end{array}$

$\begin{array}{llll}1.10 & 1.15 & 1.21 & 1.27\end{array}$

$\begin{array}{llll}1.11 & 1.18 & 1.25 & 1.32\end{array}$

$\begin{array}{llll}1.13 & 1.21 & 1.30 & 1.39\end{array}$

$\begin{array}{llll}1.16 & 1.25 & 1.35 & 1.46\end{array}$

$\begin{array}{llll}1.19 & 1.30 & 1.42 & 1.54\end{array}$

$\begin{array}{llll}1.22 & 1.34 & 1.48 & 1.63\end{array}$

$\begin{array}{llll}1.26 & 1.40 & 1.56 & 1.73\end{array}$

$\begin{array}{llll}1.29 & 1.45 & 1.63 & 1.83\end{array}$

$\begin{array}{llll}1.34 & 1.52 & 1.72 & 1.95\end{array}$

$\begin{array}{llll}1.40 & 1.60 & 1.84 & 2.10\end{array}$

$\begin{array}{llll}1.45 & 1.68 & 1.94 & 2.24\end{array}$

$\begin{array}{llll}1.49 & 1.74 & 2.03 & 2.37\end{array}$

$\begin{array}{llll}1.54 & 1.81 & 2.14 & 2.51\end{array}$

$\begin{array}{llll}1.58 & 1.88 & 2.24 & 2.66\end{array}$

$\begin{array}{llll}1.63 & 1.96 & 2.35 & 2.82\end{array}$

$\begin{array}{llll}1.67 & 2.04 & 2.47 & 2.99\end{array}$

$\begin{array}{llll}1.72 & 2.12 & 2.59 & 3.17\end{array}$

$\begin{array}{llll}1.77 & 2.20 & 2.72 & 3.36\end{array}$

$\begin{array}{llll}1.83 & 2.28 & 2.85 & 3.55\end{array}$

$\begin{array}{llll}1.87 & 2.36 & 2.98 & 3.75\end{array}$

$\begin{array}{llll}1.92 & 2.44 & 3.11 & 3.95\end{array}$

$\begin{array}{lllll}1.96 & 2.53 & 3.25 & 4.17\end{array}$

$\begin{array}{lllll}2.01 & 2.62 & 3.40 & 4.40\end{array}$

$2.01 \quad 2.62 \quad 3.40 \quad 4.40$

$\begin{array}{llll}2.06 & 2.71 & 3.55 & 4.64\end{array}$

$\begin{array}{llll}2.11 & 2.90 & 3.87 & 5.16\end{array}$ 
Table S-5. Projected fuel price indices with assumed general price inflation rates of $2 \%, 3 \%, 4 \%$, and $5 \%$, by end-use sector and fuel type.

United States Average

Projected April 1 Fuel Price Indices (April 1, 2007 = 1.00)

\begin{tabular}{|c|c|c|c|c|c|c|c|c|c|c|c|c|c|c|c|c|}
\hline \multirow[b]{3}{*}{ Year } & \multicolumn{4}{|c|}{ Electricity } & \multicolumn{4}{|c|}{ Distillate Oil } & \multicolumn{4}{|c|}{ LPG } & \multicolumn{4}{|c|}{ Natural Gas } \\
\hline & \multicolumn{4}{|c|}{ Inflation Rate } & \multicolumn{4}{|c|}{ Inflation Rate } & \multicolumn{4}{|c|}{ Inflation Rate } & & \multicolumn{2}{|c|}{ Inflation Rate } & \\
\hline & $2 \%$ & $3 \%$ & $4 \%$ & $5 \%$ & $2 \%$ & $3 \%$ & $4 \%$ & $5 \%$ & $2 \%$ & $3 \%$ & $4 \%$ & $5 \%$ & $2 \%$ & $3 \%$ & $4 \%$ & $5 \%$ \\
\hline 2008 & 1.02 & 1.03 & 1.04 & 1.05 & $1.0 \odot$ & 1.01 & 1.02 & 1.03 & 1.06 & 1.07 & 1.08 & 1.09 & $\odot .99$ & $1.0 \odot$ & 1.01 & 1.02 \\
\hline 2009 & 1.03 & 1.05 & 1.07 & 1.09 & $\odot .97$ & 0.99 & 1.01 & 1.03 & 1.06 & 1.08 & 1.10 & 1.12 & $\odot .98$ & 1.00 & 1.01 & 1.03 \\
\hline 2010 & 1.04 & 1.07 & 1.10 & 1.13 & 0.93 & 0.95 & $\odot .98$ & 1.01 & 1.07 & 1.10 & 1.13 & 1.16 & $\odot .97$ & $1.0 \odot$ & 1.03 & 1.06 \\
\hline 2011 & 1.05 & 1.09 & 1.13 & 1.17 & $\odot .89$ & $\odot .93$ & $\odot .96$ & 1.00 & 1.07 & 1.11 & 1.16 & 1.20 & 0.95 & $\odot .99$ & 1.03 & 1.07 \\
\hline 2012 & 1.06 & 1.11 & 1.16 & 1.22 & $\odot .86$ & $\odot .91$ & $\odot .95$ & 1.00 & 1.08 & 1.13 & 1.19 & 1.25 & $\odot .95$ & 1.00 & 1.05 & 1.10 \\
\hline 2013 & 1.07 & 1.14 & 1.20 & 1.28 & 0.83 & ๑. 88 & 0.93 & $\odot .99$ & 1.09 & 1.16 & 1.23 & 1.30 & $\odot .96$ & 1.01 & 1.08 & 1.14 \\
\hline 2014 & 1.09 & 1.16 & 1.25 & 1.33 & 0.83 & ๑.89 & $\odot .95$ & 1.02 & 1.11 & 1.19 & 1.28 & 1.36 & $\odot .97$ & 1.04 & 1.12 & 1.19 \\
\hline 2015 & 1.11 & 1.20 & 1.29 & 1.40 & 0.85 & $\odot .92$ & $\odot .99$ & 1.07 & 1.13 & 1.23 & 1.32 & 1.43 & $\odot .99$ & 1.07 & 1.16 & 1.25 \\
\hline 2016 & 1.13 & 1.24 & 1.35 & 1.47 & 0.87 & 0.95 & 1.04 & 1.13 & 1.16 & 1.27 & 1.38 & 1.51 & 1.02 & 1.11 & 1.21 & 1.32 \\
\hline 2017 & 1.16 & 1.28 & 1.41 & 1.55 & 0.90 & 0.99 & 1.09 & 1.20 & 1.19 & 1.31 & 1.45 & 1.59 & 1.06 & 1.16 & 1.28 & 1.41 \\
\hline 2018 & 1.19 & 1.33 & 1.48 & 1.64 & 0.92 & 1.02 & 1.14 & 1.27 & 1.22 & 1.35 & 1.51 & 1.67 & 1.08 & 1.20 & 1.33 & 1.48 \\
\hline 2019 & 1.22 & 1.37 & 1.53 & 1.72 & 0.95 & 1.07 & 1.20 & 1.35 & 1.24 & 1.39 & 1.56 & 1.75 & 1.09 & 1.23 & 1.38 & 1.55 \\
\hline 2020 & 1.24 & 1.41 & 1.59 & 1.81 & 0.98 & 1.11 & 1.26 & 1.43 & 1.27 & 1.44 & 1.63 & 1.85 & 1.12 & 1.27 & 1.44 & 1.64 \\
\hline 2021 & 1.27 & 1.45 & 1.66 & 1.90 & 1.01 & 1.16 & 1.32 & 1.51 & 1.29 & 1.48 & 1.70 & 1.94 & 1.15 & 1.32 & 1.51 & 1.72 \\
\hline 2022 & 1.29 & 1.50 & 1.73 & 2.00 & 1.04 & 1.20 & 1.39 & 1.61 & 1.32 & 1.53 & 1.77 & 2.04 & 1.18 & 1.37 & 1.58 & 1.83 \\
\hline 2023 & 1.32 & 1.54 & 1.80 & 2.10 & 1.06 & 1.24 & 1.45 & 1.69 & 1.36 & 1.59 & 1.85 & 2.16 & 1.22 & 1.43 & 1.67 & 1.94 \\
\hline 2024 & 1.35 & 1.59 & 1.88 & 2.21 & 1.09 & 1.29 & 1.52 & 1.78 & 1.39 & 1.64 & 1.94 & 2.28 & 1.26 & 1.49 & 1.76 & 2.07 \\
\hline 2025 & 1.38 & 1.64 & 1.96 & 2.33 & 1.12 & 1.33 & 1.59 & 1.88 & 1.42 & 1.69 & 2.02 & 2.39 & 1.29 & 1.54 & 1.83 & 2.18 \\
\hline 2026 & 1.41 & 1.69 & 2.03 & 2.44 & 1.15 & 1.38 & 1.66 & 1.99 & 1.45 & 1.75 & 2.10 & 2.52 & 1.32 & 1.59 & 1.91 & 2.30 \\
\hline 2027 & 1.43 & 1.74 & 2.11 & 2.56 & 1.18 & 1.44 & 1.74 & 2.11 & 1.48 & 1.80 & 2.19 & 2.65 & 1.36 & 1.65 & 2.00 & 2.43 \\
\hline 2028 & 1.46 & 1.80 & 2.20 & 2.69 & 1.21 & 1.49 & 1.83 & 2.23 & 1.52 & 1.87 & 2.29 & 2.80 & 1.40 & 1.72 & 2.11 & 2.58 \\
\hline 2029 & 1.50 & 1.86 & 2.30 & 2.84 & 1.25 & 1.55 & 1.92 & 2.37 & 1.56 & 1.93 & 2.39 & 2.95 & 1.45 & 1.79 & 2.22 & 2.74 \\
\hline 2030 & 1.53 & 1.92 & 2.39 & 2.98 & 1.29 & 1.61 & 2.01 & 2.50 & 1.59 & 1.99 & 2.49 & 3.10 & 1.48 & 1.86 & 2.32 & 2.89 \\
\hline 2031 & 1.57 & 1.98 & 2.50 & 3.14 & 1.31 & 1.66 & $2 . \odot 9$ & 2.63 & 1.62 & 2.05 & 2.59 & 3.26 & 1.52 & 1.93 & 2.43 & 3.06 \\
\hline 2032 & 1.60 & 2.05 & 2.60 & 3.31 & 1.34 & 1.71 & 2.18 & 2.77 & 1.66 & 2.12 & 2.69 & 3.42 & 1.57 & $2.0 \odot$ & 2.54 & 3.23 \\
\hline 2033 & 1.64 & 2.11 & 2.71 & 3.48 & 1.37 & 1.76 & 2.27 & 2.91 & 1.69 & 2.18 & 2.80 & 3.59 & 1.61 & 2.07 & 2.66 & 3.42 \\
\hline 2034 & 1.67 & 2.18 & 2.83 & 3.66 & 1.40 & 1.82 & 2.36 & 3.06 & 1.73 & 2.25 & 2.92 & 3.78 & 1.65 & 2.15 & 2.79 & 3.61 \\
\hline 2035 & 1.71 & 2.25 & 2.94 & 3.85 & 1.43 & 1.88 & 2.46 & 3.21 & 1.76 & 2.32 & 3.03 & 3.97 & 1.70 & 2.23 & 2.92 & 3.82 \\
\hline 2036 & 1.75 & 2.32 & 3.07 & 4.05 & 1.46 & 1.93 & 2.56 & 3.38 & 1.80 & 2.39 & 3.16 & 4.17 & 1.74 & 2.31 & 3.06 & 4.04 \\
\hline 2037 & 1.78 & 2.39 & 3.20 & 4.26 & 1.49 & 1.99 & 2.66 & 3.55 & 1.84 & 2.46 & 3.29 & 4.38 & 1.79 & 2.40 & 3.21 & 4.28 \\
\hline
\end{tabular}


Table S-5, continued. Projected fuel price indices with assumed general price inflation rates

of $2 \%, 3 \%, 4 \%$, and $5 \%$, by end-use sector and fuel type.

United States Average

Projected April 1 Fuel Price Indices (April 1, $2007=1.00$ )

\begin{tabular}{|c|c|c|c|c|c|c|c|c|c|c|c|c|c|c|c|c|c|c|c|c|}
\hline & \multicolumn{4}{|c|}{ Electricity } & \multicolumn{4}{|c|}{ Distillate $0 i l$} & \multicolumn{4}{|c|}{ Residual 0il } & \multicolumn{4}{|c|}{ Natural Gas } & \multicolumn{4}{|c|}{ Coal } \\
\hline & \multicolumn{4}{|c|}{ Inflation Rate } & \multicolumn{4}{|c|}{ Inflation Rate } & \multicolumn{4}{|c|}{ Inflation Rate } & \multicolumn{4}{|c|}{ Inflation Rate } & \multicolumn{4}{|c|}{ Inflation Rate } \\
\hline Year & $2 \%$ & $3 \%$ & $4 \%$ & $5 \%$ & $2 \%$ & $3 \%$ & $4 \%$ & $5 \%$ & $2 \%$ & $3 \%$ & $4 \%$ & $5 \%$ & $2 \%$ & $3 \%$ & $4 \%$ & $5 \%$ & $2 \%$ & $3 \%$ & $4 \%$ & $5 \%$ \\
\hline 2008 & 1.01 & 1.02 & 1.03 & 1.04 & $\odot .99$ & 1.00 & 1.01 & 1.02 & 0.95 & 0.96 & 0.97 & 0.98 & 0.99 & 1.00 & 1.01 & 1.02 & 1.03 & 1.04 & 1.05 & 1.06 \\
\hline 2009 & 1.02 & 1.04 & 1.06 & 1.08 & 0.94 & $\odot .96$ & 0.98 & 1.00 & $\odot .96$ & 0.98 & 1.00 & 1.01 & 0.96 & 0.98 & 1.00 & 1.02 & 1.05 & 1.07 & 1.09 & 1.11 \\
\hline 2010 & 1.03 & 1.06 & 1.09 & 1.12 & 0.90 & 0.93 & 0.96 & 0.99 & 0.96 & 0.99 & 1.02 & 1.05 & 0.95 & 0.98 & 1.00 & 1.03 & 1.08 & 1.11 & 1.14 & 1.17 \\
\hline 2011 & 1.02 & 1.06 & 1.10 & 1.15 & 0.87 & $\odot .90$ & 0.94 & 0.98 & 0.93 & 0.96 & 1.00 & 1.04 & 0.93 & 0.96 & 1.00 & 1.04 & 1.10 & 1.14 & 1.19 & 1.23 \\
\hline 2012 & 1.03 & 1.08 & 1.13 & 1.19 & 0.84 & $\odot .88$ & 0.92 & $\odot .97$ & 0.92 & 0.96 & 1.01 & 1.06 & 0.92 & 0.97 & 1.01 & 1.06 & 1.11 & 1.16 & 1.22 & 1.28 \\
\hline 2013 & 1.04 & 1.10 & 1.17 & 1.24 & 0.82 & $\odot .87$ & 0.92 & $\odot .97$ & 0.83 & 0.88 & 0.94 & 0.99 & 0.92 & 0.97 & 1.03 & 1.09 & 1.12 & 1.18 & 1.25 & 1.33 \\
\hline 2014 & 1.05 & 1.13 & 1.21 & 1.29 & 0.81 & $\odot .87$ & 0.93 & 1.00 & $\odot .85$ & $\odot .91$ & $\odot .98$ & 1.05 & 0.93 & $1.0 \odot$ & 1.07 & 1.14 & 1.13 & 1.21 & 1.29 & 1.38 \\
\hline 2015 & 1.07 & 1.16 & 1.25 & 1.35 & 0.83 & 0.90 & 0.97 & 1.05 & $0.9 \odot$ & 0.97 & 1.05 & 1.14 & 0.94 & 1.02 & 1.10 & 1.19 & 1.15 & 1.24 & 1.34 & 1.45 \\
\hline 2016 & 1.10 & 1.20 & 1.31 & 1.43 & 0.85 & 0.93 & 1.02 & 1.11 & $\odot .92$ & 1.01 & 1.10 & 1.20 & 0.97 & 1.06 & 1.15 & 1.26 & 1.17 & 1.28 & 1.39 & 1.52 \\
\hline 2017 & 1.13 & 1.25 & 1.38 & 1.52 & $\odot .88$ & $\odot .97$ & 1.07 & 1.17 & 0.95 & 1.05 & 1.16 & 1.27 & 1.01 & 1.11 & 1.22 & 1.34 & 1.18 & 1.30 & 1.43 & 1.58 \\
\hline 2018 & 1.17 & 1.30 & 1.45 & 1.61 & 0.90 & 1.01 & 1.12 & 1.24 & 0.99 & 1.10 & 1.23 & 1.36 & 1.02 & 1.14 & 1.27 & 1.41 & 1.20 & 1.33 & 1.48 & 1.65 \\
\hline 2019 & 1.19 & 1.34 & 1.50 & 1.69 & $\odot .94$ & 1.06 & 1.19 & 1.33 & 1.05 & 1.18 & 1.32 & 1.48 & 1.04 & 1.16 & 1.31 & 1.47 & 1.22 & 1.37 & 1.54 & 1.72 \\
\hline 2020 & 1.22 & 1.38 & 1.57 & 1.77 & 0.97 & 1.10 & 1.25 & 1.41 & 1.09 & 1.24 & 1.40 & 1.59 & 1.06 & 1.21 & 1.37 & 1.55 & 1.25 & 1.42 & 1.61 & 1.82 \\
\hline 2021 & 1.24 & 1.43 & 1.63 & 1.87 & $1 . \odot \odot$ & 1.14 & 1.31 & 1.49 & 1.13 & 1.30 & 1.49 & 1.70 & 1.08 & 1.24 & 1.42 & 1.62 & 1.28 & 1.47 & 1.68 & 1.92 \\
\hline 2022 & 1.27 & 1.47 & 1.70 & 1.96 & 1.03 & 1.20 & 1.38 & 1.60 & 1.18 & 1.37 & 1.58 & 1.83 & 1.11 & 1.29 & 1.49 & 1.72 & 1.31 & 1.52 & 1.75 & 2.02 \\
\hline 2023 & 1.30 & 1.52 & 1.77 & 2.06 & 1.05 & 1.22 & 1.43 & 1.67 & 1.19 & 1.39 & 1.63 & 1.90 & 1.15 & 1.35 & 1.57 & 1.83 & 1.34 & 1.57 & 1.83 & 2.13 \\
\hline 2024 & 1.33 & 1.57 & 1.85 & 2.18 & 1.08 & 1.27 & 1.50 & 1.76 & 1.23 & 1.45 & 1.71 & 2.01 & 1.19 & 1.40 & 1.65 & 1.94 & 1.38 & 1.62 & 1.91 & 2.25 \\
\hline 2025 & 1.36 & 1.62 & 1.93 & 2.29 & 1.11 & 1.33 & 1.58 & 1.88 & 1.23 & 1.47 & 1.75 & 2.07 & 1.21 & 1.45 & 1.72 & 2.05 & 1.41 & 1.68 & 2.00 & 2.37 \\
\hline 2026 & 1.39 & 1.67 & 2.00 & 2.40 & 1.14 & 1.37 & 1.65 & 1.98 & 1.26 & 1.52 & 1.83 & 2.19 & 1.24 & 1.49 & 1.79 & 2.15 & 1.44 & 1.74 & 2.09 & 2.50 \\
\hline 2027 & 1.41 & 1.72 & 2.08 & 2.52 & 1.18 & 1.43 & 1.74 & 2.10 & 1.31 & 1.59 & 1.93 & 2.33 & 1.27 & 1.55 & 1.88 & 2.27 & 1.49 & 1.81 & 2.19 & 2.65 \\
\hline 2028 & 1.44 & 1.77 & 2.17 & 2.65 & 1.22 & 1.50 & 1.84 & 2.24 & 1.35 & 1.65 & 2.02 & 2.47 & 1.31 & 1.61 & 1.98 & 2.42 & 1.52 & 1.87 & 2.29 & 2.80 \\
\hline 2029 & 1.47 & 1.83 & 2.26 & 2.79 & 1.26 & 1.56 & 1.93 & 2.38 & 1.34 & 1.67 & 2.06 & 2.54 & 1.35 & 1.68 & 2.08 & 2.56 & 1.57 & 1.94 & 2.40 & 2.96 \\
\hline 2030 & 1.50 & 1.88 & 2.35 & 2.93 & 1.29 & 1.62 & 2.02 & 2.52 & 1.37 & 1.72 & 2.14 & 2.67 & 1.39 & 1.74 & 2.17 & 2.71 & 1.61 & 2.01 & 2.51 & 3.13 \\
\hline 2031 & 1.54 & 1.94 & 2.45 & 3.08 & 1.32 & 1.67 & 2.11 & 2.66 & 1.40 & 1.78 & 2.24 & 2.82 & 1.43 & 1.81 & 2.28 & 2.87 & 1.64 & 2.08 & 2.62 & 3.30 \\
\hline 2032 & 1.57 & 2.01 & 2.55 & 3.24 & 1.35 & 1.73 & 2.20 & 2.79 & 1.44 & 1.83 & 2.33 & 2.97 & 1.47 & 1.88 & 2.39 & 3.04 & 1.68 & 2.15 & 2.74 & 3.48 \\
\hline 2033 & 1.61 & 2.07 & 2.66 & 3.41 & 1.38 & 1.78 & 2.29 & 2.94 & 1.47 & 1.89 & 2.43 & 3.12 & 1.51 & 1.95 & 2.51 & 3.21 & 1.73 & 2.22 & 2.86 & 3.67 \\
\hline 2034 & 1.64 & 2.13 & 2.77 & 3.59 & 1.41 & 1.84 & 2.38 & 3.08 & 1.50 & 1.95 & 2.54 & 3.28 & 1.56 & 2.03 & 2.63 & 3.40 & 1.77 & 2.30 & 2.99 & 3.87 \\
\hline 2035 & 1.68 & 2.20 & 2.89 & 3.77 & 1.44 & 1.89 & 2.48 & 3.24 & 1.53 & 2.01 & 2.64 & 3.45 & 1.60 & 2.10 & 2.76 & 3.60 & 1.81 & 2.38 & 3.12 & 4.08 \\
\hline 2036 & 1.71 & 2.27 & 3.00 & 3.97 & 1.47 & 1.95 & 2.58 & 3.41 & 1.57 & 2.08 & 2.75 & 3.63 & 1.65 & 2.19 & 2.89 & 3.82 & 1.86 & 2.46 & 3.26 & 4.30 \\
\hline 2037 & 1.75 & 2.34 & 3.13 & 4.17 & 1.50 & 2.01 & 2.69 & 3.59 & 1.60 & 2.15 & 2.87 & 3.82 & 1.69 & 2.27 & 3.03 & 4.04 & 1.90 & 2.55 & 3.40 & 4.54 \\
\hline
\end{tabular}


Table S-5, continued. Projected fuel price indices with assumed general price inflation rates of $2 \%, 3 \%, 4 \%$, and $5 \%$, by end-use sector and fuel type.

United States Average

Projected April 1 Fuel Price Indices (April 1, $2007=1.00$ )

\begin{tabular}{|c|c|c|c|c|c|c|c|c|c|c|c|c|c|c|c|c|c|c|c|c|}
\hline \multirow[b]{3}{*}{ Year } & \multicolumn{4}{|c|}{ Electricity } & \multicolumn{4}{|c|}{ Distillate 0il } & \multicolumn{4}{|c|}{ Residual oil } & \multicolumn{4}{|c|}{ Natural Gas } & \multicolumn{4}{|c|}{ Coal } \\
\hline & \multicolumn{4}{|c|}{ Inflation Rate } & \multicolumn{4}{|c|}{ Inflation Rate } & \multicolumn{4}{|c|}{ Inflation Rate } & \multicolumn{4}{|c|}{ Inflation Rate } & \multicolumn{4}{|c|}{ Inflation Rate } \\
\hline & $2 \%$ & $3 \%$ & $4 \%$ & $5 \%$ & $2 \%$ & $3 \%$ & $4 \%$ & $5 \%$ & $2 \%$ & $3 \%$ & $4 \%$ & $5 \%$ & $2 \%$ & $3 \%$ & $4 \%$ & $5 \%$ & $2 \%$ & $3 \%$ & $4 \%$ & $5 \%$ \\
\hline 2008 & 1.03 & 1.04 & 1.05 & 1.06 & 0.97 & 0.98 & 0.99 & 1.00 & 1.04 & 1.05 & 1.06 & 1.07 & 1.01 & 1.02 & 1.03 & 1.04 & 1.03 & 1.04 & 1.05 & 1.06 \\
\hline 2009 & 1.04 & 1.06 & 1.08 & 1.10 & 0.92 & 0.94 & 0.96 & 0.98 & 1.02 & 1.04 & 1.06 & 1.09 & 0.97 & 0.99 & 1.01 & 1.03 & 1.05 & 1.07 & 1.09 & 1.11 \\
\hline 2010 & 1.03 & 1.06 & 1.09 & 1.13 & 0.90 & 0.93 & 0.96 & 0.99 & 1.00 & 1.03 & 1.06 & 1.09 & 0.94 & 0.96 & 0.99 & 1.02 & 1.07 & 1.10 & 1.13 & 1.17 \\
\hline 2011 & 1.01 & 1.05 & 1.09 & 1.14 & 0.89 & 0.92 & 0.96 & 1.00 & 0.97 & 1.01 & 1.05 & 1.09 & 0.90 & 0.93 & 0.97 & 1.01 & 1.09 & 1.14 & 1.18 & 1.23 \\
\hline 2012 & 1.00 & 1.05 & 1.10 & 1.16 & 0.86 & 0.91 & 0.95 & 1.00 & 0.95 & 1.00 & 1.05 & 1.10 & 0.88 & 0.93 & $\odot .97$ & 1.02 & 1.10 & 1.16 & 1.22 & 1.28 \\
\hline 2013 & 1.01 & 1.07 & 1.13 & 1.20 & $\odot .86$ & 0.91 & 0.96 & 1.02 & $\odot .92$ & 0.98 & 1.04 & 1.10 & 0.87 & 0.93 & 0.98 & 1.04 & 1.12 & 1.18 & 1.25 & 1.33 \\
\hline 2014 & 1.02 & 1.09 & 1.16 & 1.25 & 0.85 & 0.91 & 0.98 & 1.05 & 0.92 & 0.99 & 1.06 & 1.13 & 0.89 & 0.95 & 1.01 & 1.08 & 1.14 & 1.22 & 1.30 & 1.39 \\
\hline 2015 & 1.03 & 1.12 & 1.21 & 1.30 & 0.87 & 0.94 & 1.01 & 1.10 & 0.95 & 1.03 & 1.11 & 1.20 & 0.90 & $\odot .97$ & 1.05 & 1.13 & 1.16 & 1.25 & 1.35 & 1.46 \\
\hline 2016 & 1.06 & 1.16 & 1.27 & 1.38 & 0.90 & 0.98 & 1.07 & 1.17 & 0.97 & 1.06 & 1.16 & 1.26 & 0.93 & 1.01 & 1.10 & 1.20 & 1.17 & 1.28 & 1.40 & 1.52 \\
\hline 2017 & 1.10 & 1.21 & 1.33 & 1.47 & 0.92 & 1.02 & 1.12 & 1.23 & 1.01 & 1.11 & 1.22 & 1.34 & 0.97 & 1.07 & 1.18 & 1.30 & 1.20 & 1.32 & 1.45 & 1.60 \\
\hline 2018 & 1.13 & 1.26 & 1.40 & 1.56 & $\odot .95$ & 1.06 & 1.17 & 1.30 & 1.04 & 1.15 & 1.28 & 1.42 & 0.99 & 1.10 & 1.23 & 1.37 & 1.22 & 1.35 & 1.50 & 1.67 \\
\hline 2019 & 1.16 & 1.30 & 1.46 & 1.64 & 0.99 & 1.11 & 1.25 & 1.40 & 1.08 & 1.21 & 1.36 & 1.53 & 1.01 & 1.13 & 1.27 & 1.42 & 1.24 & 1.39 & 1.57 & 1.76 \\
\hline 2020 & 1.18 & 1.34 & 1.52 & 1.72 & 1.01 & 1.15 & 1.31 & 1.48 & 1.12 & 1.27 & 1.44 & 1.63 & 1.03 & 1.17 & 1.33 & 1.50 & 1.26 & 1.44 & 1.63 & 1.84 \\
\hline 2021 & 1.21 & 1.39 & 1.59 & 1.82 & 1.04 & 1.19 & 1.37 & 1.56 & 1.16 & 1.33 & 1.52 & 1.74 & 1.05 & 1.21 & 1.38 & 1.58 & 1.30 & 1.49 & 1.70 & 1.94 \\
\hline 2022 & 1.24 & 1.43 & 1.66 & 1.91 & 1.08 & 1.25 & 1.45 & 1.67 & 1.21 & 1.39 & 1.61 & 1.86 & 1.09 & 1.26 & 1.46 & 1.68 & 1.33 & 1.54 & 1.78 & 2.06 \\
\hline 2023 & 1.27 & 1.48 & 1.73 & 2.01 & 1.09 & 1.28 & 1.49 & 1.74 & 1.22 & 1.43 & 1.67 & 1.94 & 1.13 & 1.32 & 1.55 & 1.80 & 1.36 & 1.59 & 1.86 & 2.16 \\
\hline 2024 & 1.30 & 1.53 & 1.81 & 2.13 & 1.12 & 1.32 & 1.56 & 1.84 & 1.26 & 1.48 & 1.75 & 2.06 & 1.18 & 1.39 & 1.64 & 1.93 & 1.39 & 1.65 & 1.94 & 2.28 \\
\hline 2025 & 1.33 & 1.58 & 1.88 & 2.24 & 1.17 & 1.39 & 1.66 & 1.97 & 1.28 & 1.53 & 1.82 & 2.16 & 1.20 & 1.43 & 1.71 & 2.03 & 1.43 & 1.70 & 2.03 & 2.41 \\
\hline 2026 & 1.35 & 1.63 & 1.96 & 2.35 & 1.19 & 1.44 & 1.73 & 2.07 & 1.32 & 1.58 & 1.90 & 2.28 & 1.23 & 1.48 & 1.78 & 2.14 & 1.46 & 1.76 & 2.12 & 2.54 \\
\hline 2027 & 1.38 & 1.68 & 2.03 & 2.46 & 1.23 & 1.50 & 1.82 & 2.20 & 1.36 & 1.65 & 2.00 & 2.42 & 1.27 & 1.54 & 1.87 & 2.27 & 1.50 & 1.82 & 2.21 & 2.68 \\
\hline 2028 & 1.41 & 1.73 & 2.12 & 2.59 & 1.28 & 1.57 & 1.93 & 2.36 & 1.39 & 1.71 & 2.09 & 2.56 & 1.31 & 1.61 & 1.98 & 2.42 & 1.54 & 1.89 & 2.31 & 2.82 \\
\hline 2029 & 1.44 & 1.79 & 2.21 & 2.73 & 1.31 & 1.63 & 2.02 & 2.49 & 1.42 & 1.76 & 2.18 & 2.69 & 1.36 & 1.69 & 2.08 & 2.57 & 1.57 & 1.95 & 2.41 & 2.98 \\
\hline 2030 & 1.47 & 1.85 & 2.30 & 2.87 & 1.36 & 1.70 & 2.12 & 2.64 & 1.46 & 1.83 & 2.28 & 2.84 & 1.40 & 1.75 & 2.19 & 2.73 & 1.61 & 2.02 & 2.52 & 3.14 \\
\hline 2031 & 1.51 & 1.91 & 2.40 & 3.02 & 1.39 & 1.75 & 2.21 & 2.78 & 1.49 & 1.89 & 2.38 & 2.99 & 1.44 & 1.82 & 2.30 & 2.89 & 1.65 & 2.09 & 2.63 & 3.31 \\
\hline 2032 & 1.54 & 1.97 & 2.50 & 3.18 & 1.42 & 1.81 & 2.30 & 2.92 & 1.52 & 1.95 & 2.48 & 3.15 & 1.49 & 1.90 & 2.42 & 3.07 & 1.69 & 2.16 & 2.75 & 3.49 \\
\hline 2033 & 1.58 & 2.03 & 2.61 & 3.35 & 1.45 & 1.86 & 2.40 & 3.07 & 1.56 & 2.01 & 2.58 & 3.31 & 1.54 & 1.98 & 2.54 & 3.26 & 1.73 & 2.23 & 2.87 & 3.68 \\
\hline 2034 & 1.61 & 2.10 & 2.72 & 3.52 & 1.48 & 1.92 & 2.49 & 3.23 & 1.59 & 2.07 & 2.69 & 3.48 & 1.58 & 2.06 & 2.68 & 3.47 & 1.78 & 2.31 & 3.00 & 3.88 \\
\hline 2035 & 1.65 & 2.16 & 2.84 & 3.71 & 1.51 & 1.98 & 2.60 & 3.40 & 1.63 & 2.14 & 2.80 & 3.66 & 1.63 & 2.15 & 2.82 & 3.68 & 1.82 & 2.39 & 3.13 & 4.10 \\
\hline 2036 & 1.68 & 2.23 & 2.96 & 3.90 & 1.54 & 2.04 & 2.70 & 3.57 & 1.66 & 2.20 & 2.91 & 3.85 & 1.69 & 2.24 & 2.97 & 3.92 & 1.86 & 2.47 & 3.27 & 4.32 \\
\hline 2037 & 1.72 & 2.31 & 3.08 & 4.11 & 1.57 & 2.11 & 2.82 & 3.75 & 1.70 & 2.27 & 3.04 & 4.05 & 1.74 & 2.34 & 3.12 & 4.16 & 1.91 & 2.56 & 3.42 & 4.55 \\
\hline
\end{tabular}

